Supporting Information for

\title{
Importance of the Electron Correlation and Dispersion Corrections in Calculations Involving Enamines, Hemiaminals, and Aminals. Comparison of B3LYP, M06-2X, MP2, and CCSD Results with Experimental Data
}

\author{
Alejandro Castro-Alvarez, Héctor Carneros, Dani Sánchez, and Jaume Vilarrasa* \\ Departament de Química Orgànica, Facultat de Química, Universitat de Barcelona, Diagonal 645, \\ 08028 Barcelona, Catalonia, Spain
}

Table 1. Calcd vs. Exptl $\Delta G^{\mathrm{o}}$ Values for the Equilibrium between Enamines 1a and 2a and Their Ketones ....... 2 Addendum to Table 1. Reaction of Enamine 1a with Cyclopentanone ............................................. 4

Table 2. Calcd vs. Exptl $\Delta G^{\text {o }}$ Values for the Formation of Enamine 5a from 5 ................................................ 5

Addendum to Table 2. Formation of Enamine 4a from Aldehyde 4 ............................................................... 7

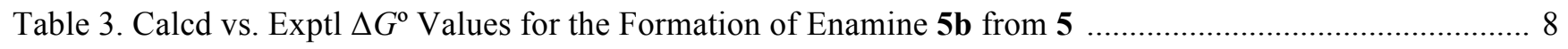

Table 4. Calcd $\Delta G^{\circ}$ Values for the Formation of Hemiaminal HA-4a .......................................................... 10

Addendum to Table 4. Formation of Hemiaminal HA-3a from Cyclopentanone (3) ................................... 11

Addendum to Table 4-bis. Formation of Hemiaminal HA-6a from Pivalaldehyde (6) ................................ 12

Table 5. Calcd $\Delta G^{\mathrm{o}}$ Values for the Reactions of Methyl Glyoxylate with Pyrrolidine ....................................... 13

Addendum to Table 5. Formation of Hemiaminal HA-7b from Methyl Glyoxylate and Prolinol b ....... 14

Table 6. Calcd vs Exptl $\Delta G^{\mathrm{o}}$ Values for the Formation of Aminal 5aa from 5a and Pyrrolidine ....................... 15

Table 7. Calcd vs. Exptl $\Delta G^{\circ}$ Values for the Exchange of a Cyclohexenyl Group between Secondary Amines......... 16

Table 8. Calcd vs. Exptl $\Delta G^{\mathrm{o}}$ Values for the Exchange of an Alkenyl Group between Secondary Amines ............... 16

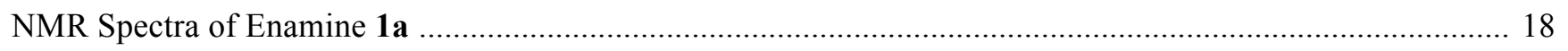

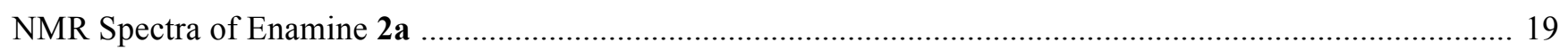

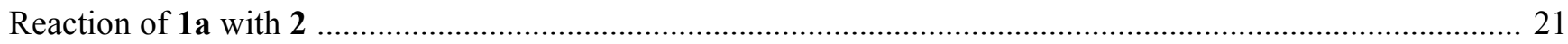

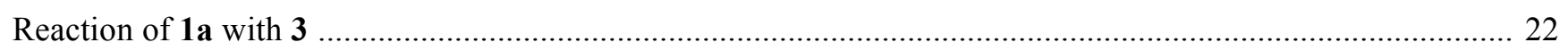

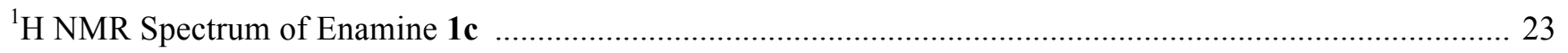

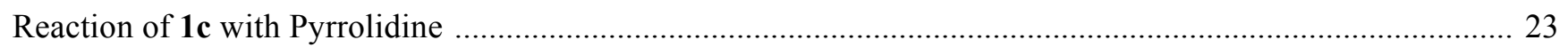

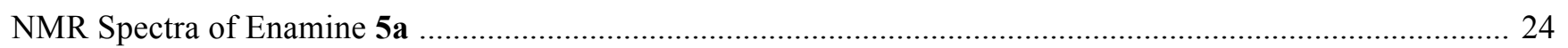

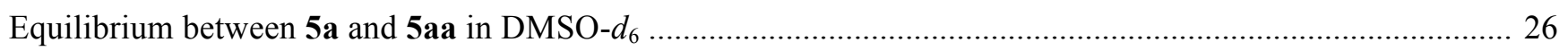

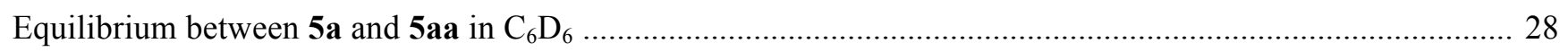

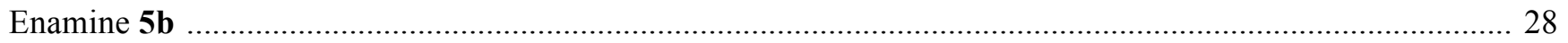

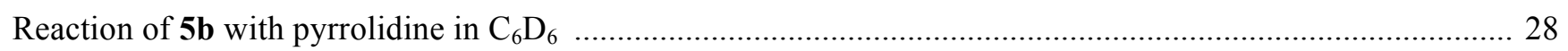

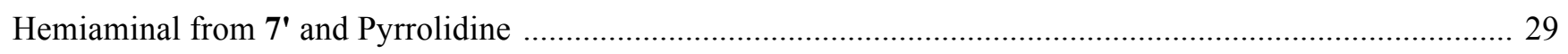

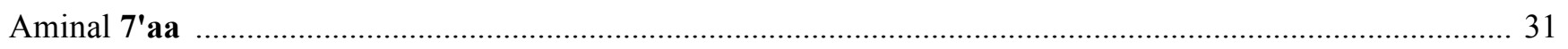

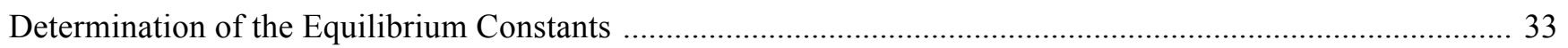




\section{Table 1. Calcd vs. Exptl $\Delta G^{\circ}$ Values for the Equilibrium between Enamines 1a and $2 \mathrm{a}$ and Their Ketones}

Calculated energies for individual molecules are in au or hartrees $(1 \mathrm{au}=627.5 \mathrm{kcal} / \mathrm{mol})$ while reaction energies and $/$ or $\Delta G^{\circ}$ values are given in $\mathrm{kcal} / \mathrm{mol}\left(1 \mathrm{kcal} / \mathrm{mol}=4.184 \mathrm{~kJ} \cdot \mathrm{mol}^{-1}\right)$. Gaussian 09 , Revision D.01, 2013, was used everywhere.* The ORCA 3.0.2 program** was employed in Tables 4-7 for the sake of comparison. Solvent effects were considered through single-point energy calculations at every level of theory, with the SMD model (Solvent Model based on Density); other implicit solvation models have been tested (see next pages).

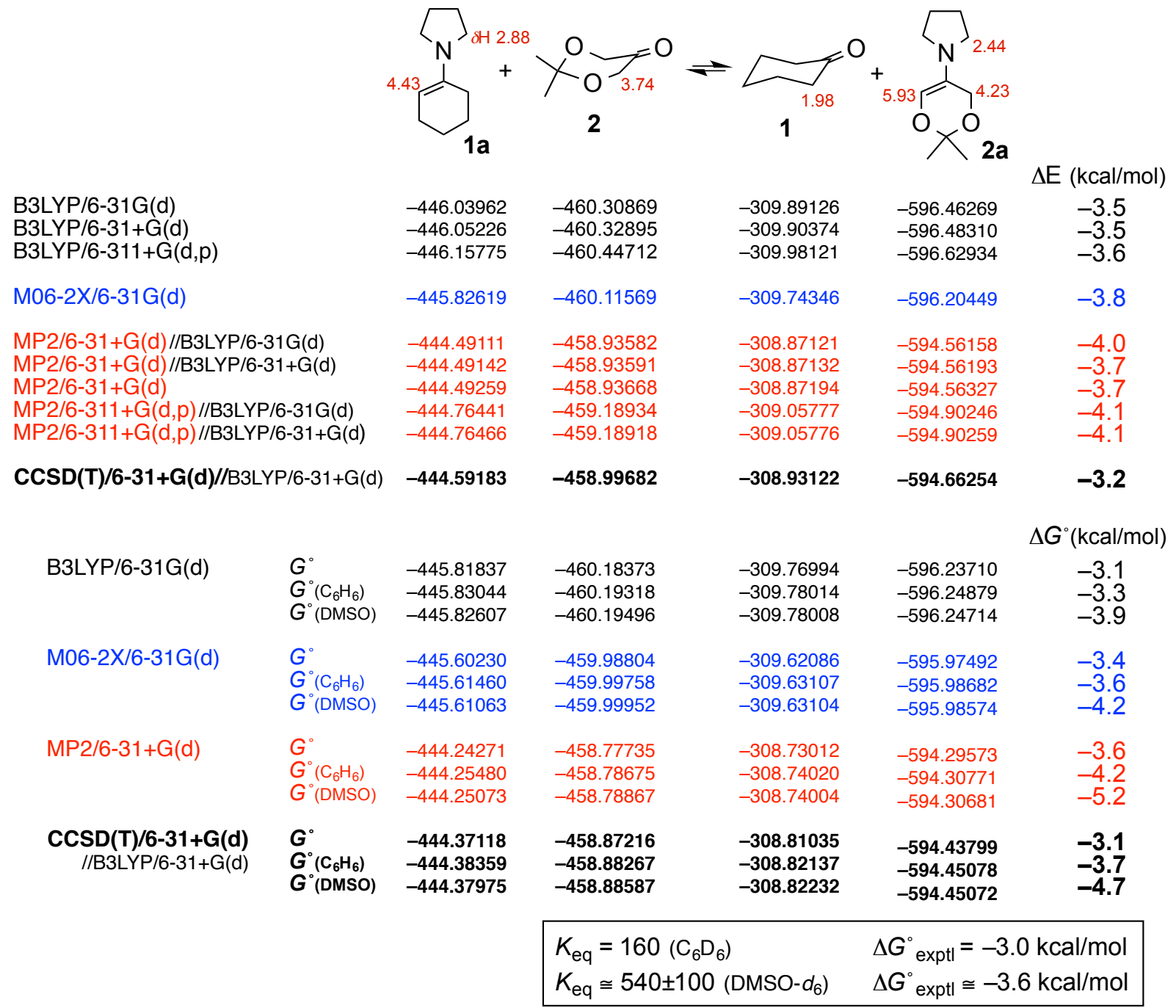

\footnotetext{
* Gaussian 09, Revision D.01. Frisch, M. J.; Trucks, G. W.; Schlegel, H. B.; Scuseria, G. E.; Robb, M. A.; Cheeseman, J. R.; Montgomery, Jr., J. A.; Vreven, T.; Kudin, K. N.; Burant, J. C.; Millam, J. M.; Iyengar, S. S.; Tomasi, J.; Barone, V.; Mennucci, B.; Cossi, M.; Scalmani, G.; Rega, N.; Petersson, G. A.; Nakatsuji, H.; Hada, M.; Ehara, M.; Toyota, K.; Fukuda, R.; Hasegawa, J.; Ishida, M.; Nakajima, T.; Honda, Y.; Kitao, O.; Nakai, H.; Klene, M.; Li, X.; Knox, J. E.; Hratchian, H. P.; Cross, J. B.; Bakken, V.; Adamo, C.; Jaramillo, J.; Gomperts, R.; Stratmann, R. E.; Yazyev, O.; Austin, A. J.; Cammi, R.; Pomelli, C.; Ochterski, J. W.; Ayala, P. Y.; Morokuma, K.; Voth, G. A.; Salvador, P.; Dannenberg, J. J.; Zakrzewski, V. G.; Dapprich, S.; Daniels, A. D.; Strain, M. C.; Farkas, O.; Malick, D. K.; Rabuck, A. D.; Raghavachari, K.; Foresman, J. B.; Ortiz, J. V.; Cui, Q.; Baboul, A. G.; Clifford, S.; Cioslowski, J.; Stefanov, B. B.; Liu, G.; Liashenko, A.; Piskorz, P.; Komaromi, I.; Martin, R. L.; Fox, D. J.; Keith, T.; Al-Laham, M. A.; Peng, C. Y.; Nanayakkara, A.; Challacombe, M.; Gill, P. M. W.; Johnson, B.; Chen, W.; Wong, M. W.; Gonzalez, C.; and Pople, J. A.; Gaussian, Inc., Wallingford CT, 2004.

** ORCA 3.0.2. (a) Neese, F. The ORCA program system, Wiley Interdiscip. Rev.: Comput. Mol. Sci. 2012, 2, 73-78. (b) Neese, F.; Wennmohs, F.; et al., Max-Planck-Institut für Chemische Energiekonversion, Mülheim (www.cec.mpg.de).
} 
B3LYP/6-31G(d)

- without scaling factor

— with scaling factor $(0.960)^{*}$

MP2/6-31G(d)

— without scaling factor

— with scaling factor $(0.943)^{*}$

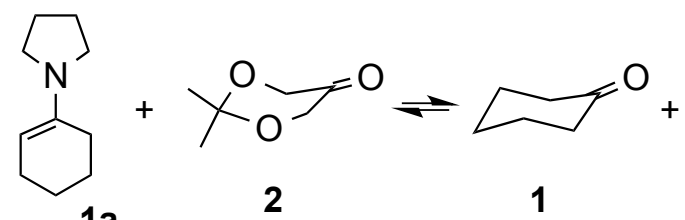

$1 a$

$\begin{array}{ll}-445.81837 & -460.18373 \\ -445.82907 & -460.18996\end{array}$

$-309.76994$

$-309.77617$

$-596.23710$

$-596.24822$

$\Delta G^{\circ}(\mathrm{kcal} / \mathrm{mol})$

$-3.1$

$-444.24271-458.77735$

$-444.25814-458.78692$
$-594.29573$

$-594.31171$
$-3.6$

$-3.6$

Differences among continuum solvation models, at the B3LYP/6-31G(d) level

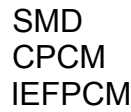

$\begin{array}{ll}-446.04733 & -460.31991 \\ -446.04178 & -460.31545 \\ -446.04174 & -460.31538\end{array}$

-309.90139
-309.89695

$-309.89689$
$-596.47272$

$-596.46829$

$-596.46820$
$\Delta \mathrm{E}(\mathrm{kcal} / \mathrm{mol})$

$-4.3$

$-5.0$

\footnotetext{
* http://cccbdb.nist.gov/vibscalejust.asp (National Institute of Standards and Technology, Computational Chemistry Comparison and Benchmark Database, Release 16a, August 2013, Johnson III, R. D., Ed.).
}

Cartesian coordinates of the stationary points at the B3LYP/6-31+G(d) level<smiles>C1=C(N2CCCC2)CCC1</smiles>

$1 a$

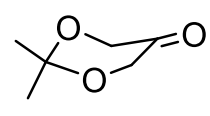

2

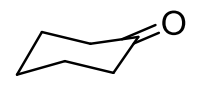

1

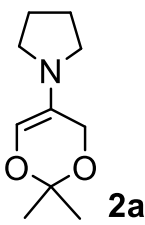

$2 a$

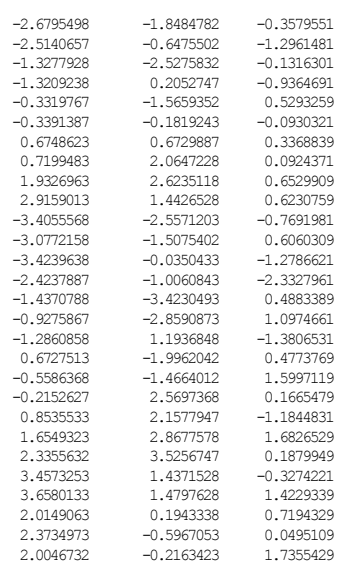

$\begin{array}{rrr}0.0709321 & 0.6028852 & -1.0107548 \\ -0.7217889 & -0.0458378 & -0.0063718 \\ 0.1287931 & -0.5904298 & 1.0123902 \\ 1.3162481 & 0.1512392 & 1.2686392 \\ 2.1882511 & 0.1375002 & 0.0196012 \\ -1.6402349 & 1.0316852 & 0.5581612 \\ -1.4875809 & -1.2294918 & -0.5855478 \\ 1.1125241 & 1.1894332 & 1.5651932 \\ 1.8332691 & -0.3451858 & 2.0896382 \\ -2.3054069 & 0.5982402 & 1.3068872 \\ -1.0577189 & 1.8313612 & 1.0193342 \\ -2.2393479 & 1.4684992 & -0.2429628 \\ -2.0395119 & -1.7402368 & 0.2057302 \\ -2.1902699 & -0.8819628 & -1.3448938 \\ -0.8021079 & -1.9485758 & -1.0380238 \\ 1.3471381 & 0.0179362 & -1.2450238 \\ 1.2820851 & -1.0366848 & -1.5463418 \\ 1.8122651 & 0.5781902 & -2.0559388 \\ 3.3924621 & 0.2114362 & 0.0302852\end{array}$

-0.8581284
-1.3402134
-0.4785244
1.00147286
1.5067186
1.1570086
1.6465666
0.630176
1.0083496
0.7463236
2.5261696
-2.3883224
-1.2993734
-1.4610994
-0.9961524
-0.6011214
-0.8130404

-0.0426657
0.3052793
-0.3841137
-0.0331387
-0.2713007
1.0341043
-0.5992727
0.3118903
-0.0292077
1.4038973
-0.8826337
0.0142383
1.3928703
0.4753883
-1.1167157
-1.4707327
-0.1084267
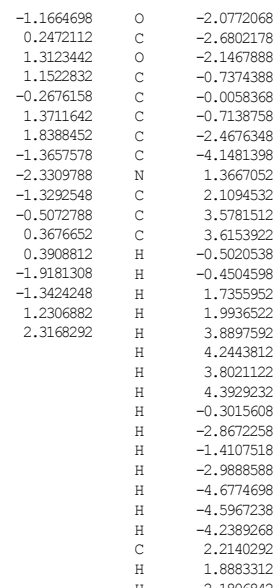

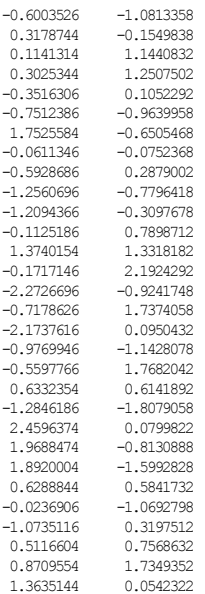




\begin{tabular}{|c|c|c|c|c|c|c|}
\hline & & $1 \mathrm{a}$ & 3 & 1 & $3 a$ & $\begin{array}{c}\text { reaction } \\
\text { energy } \\
(\mathrm{kcal} / \mathrm{mol})\end{array}$ \\
\hline B3LYP/6-31G(d) & $\begin{array}{l}\mathrm{E} \\
G^{\circ} \\
G^{\circ} \text { (DMSO) }\end{array}$ & $\begin{array}{l}-446.03962 \\
-445.81837 \\
-445.82607\end{array}$ & $\begin{array}{l}-270.57283 \\
-270.48004 \\
-270.48984\end{array}$ & $\begin{array}{l}-309.89126 \\
-309.76994 \\
-309.78008\end{array}$ & $\begin{array}{l}-406.72231 \\
-406.53004 \\
-406.53742\end{array}$ & $\begin{array}{l}-0.7 \\
-1.0 \\
-1.0\end{array}$ \\
\hline B3LYP/6-311+G(d,p) & $\begin{array}{l}\mathrm{E} \\
G^{\circ} \\
G^{\circ}(\mathrm{DMSO})\end{array}$ & $\begin{array}{l}-446.15775 \\
-445.93888 \\
-445.94750\end{array}$ & $\begin{array}{l}-270.65271 \\
-270.56102 \\
-270.57275\end{array}$ & $\begin{array}{l}-309.98121 \\
-309.86132 \\
-309.87345\end{array}$ & $\begin{array}{l}-406.83054 \\
-406.64019 \\
-406.64843\end{array}$ & $\begin{array}{l}-0.8 \\
-1.0 \\
-1.0\end{array}$ \\
\hline M06-2X/6-31G(d) & $\begin{array}{l}\mathrm{E} \\
G^{\circ} \\
G^{\circ}(\mathrm{DMSO})\end{array}$ & $\begin{array}{l}-445.82619 \\
-445.60230 \\
-445.61063\end{array}$ & $\begin{array}{l}-270.44443 \\
-270.35046 \\
-270.36040\end{array}$ & $\begin{array}{l}-309.74346 \\
-309.62086 \\
-309.63104\end{array}$ & $\begin{array}{l}-406.52727 \\
-406.33213 \\
-406.34003\end{array}$ & $\begin{array}{l}-0.1 \\
-0.1 \\
-0.0\end{array}$ \\
\hline M06-2X/6-311+G(d,p) & $\begin{array}{l}\mathrm{E}^{\circ} \\
\mathrm{G}^{\circ} \text { (DMSO) }\end{array}$ & $\begin{array}{l}-445.94623 \\
-445.72463 \\
-445.73414\end{array}$ & $\begin{array}{l}-270.52453 \\
-270.43168 \\
-270.44331\end{array}$ & $\begin{array}{l}-309.83413 \\
-309.71289 \\
-309.72486\end{array}$ & $\begin{array}{l}-406.63705 \\
-406.44441 \\
-406.45345\end{array}$ & $\begin{array}{l}-0.3 \\
-0.6 \\
-0.5\end{array}$ \\
\hline $\begin{array}{l}\text { MP2/6-31+G(d) } \\
\text { //B3LYP/6-31G(d) }\end{array}$ & $\begin{array}{l}E \\
G^{\circ} \\
G^{\circ} \text { (DMSO) }\end{array}$ & $\begin{array}{l}-444.49111 \\
-444.26985 \\
-444.27872\end{array}$ & $\begin{array}{l}-269.69790 \\
-269.60511 \\
-269.61626\end{array}$ & $\begin{array}{l}-308.87121 \\
-308.74990 \\
-308.76151\end{array}$ & $\begin{array}{l}-405.31891 \\
-405.12664 \\
-405.13513\end{array}$ & $\begin{array}{l}-0.7 \\
-1.0 \\
-1.0\end{array}$ \\
\hline $\begin{array}{l}\text { MP2/6-311+G(d,p) } \\
\text { //B3LYP/6-311+G(d,p) }\end{array}$ & $\begin{array}{l}E \\
G^{\circ} \\
G^{\circ} \text { (DMSO) }\end{array}$ & $\begin{array}{l}-444.76465 \\
-444.54578 \\
-444.55443\end{array}$ & $\begin{array}{l}-269.85601 \\
-269.76433 \\
-269.77486\end{array}$ & $\begin{array}{l}-309.05771 \\
-308.93781 \\
-308.94881\end{array}$ & $\begin{array}{l}-405.56397 \\
-405.37362 \\
-405.38185\end{array}$ & $\begin{array}{l}-0.6 \\
-0.8 \\
-0.9\end{array}$ \\
\hline \multirow[t]{2}{*}{$\begin{array}{l}\text { MP2/6-311+G(d,p) } \\
\text { //M06-2X/6-311+G(d,p) }\end{array}$} & \multirow[t]{2}{*}{$\begin{array}{l}E \\
G^{\circ} \\
G^{\circ}(\mathrm{DMSO})\end{array}$} & \multirow[t]{2}{*}{$\begin{array}{l}-444.76522 \\
-444.54363 \\
-444.55219\end{array}$} & $\begin{array}{l}-269.85597 \\
-269.76311 \\
-269.77368\end{array}$ & $\begin{array}{l}-309.05771 \\
-308.93648 \\
-308.94747\end{array}$ & $\begin{array}{l}-405.56455 \\
-405.37191 \\
-405.38008\end{array}$ & $\begin{array}{l}-0.7 \\
-1.0 \\
-1.1\end{array}$ \\
\hline & & & $\begin{array}{l}=2.0 \pm 0 \\
=2.1 \pm 0\end{array}$ & $\begin{array}{ll} & \Delta \\
\left.-d_{6}\right) & \Delta\end{array}$ & $\begin{array}{l}=-0.4 \pm 0 \\
=-0.4 \pm 0\end{array}$ & $\begin{array}{l}\text { al/mol } \\
\text { al/mol }\end{array}$ \\
\hline
\end{tabular}

Cartesian coordinates of the stationary points, at the B3LYP/6-311+G(d,p) level

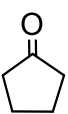

3
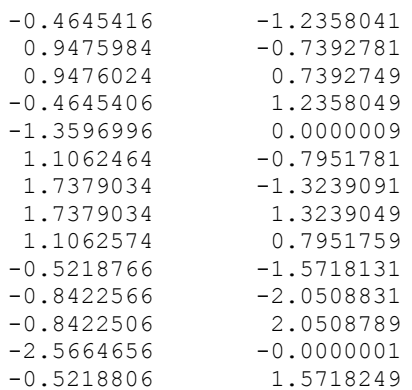

0.1217654 $-0.2257966$

0.2257914

$-0.1217606$

$-0.0000006$

$-1.3080286$

0.2485864

$-0.2485986$

1. 3080224

1. 1648724

$-0.4979586$

0.4979734

$-0.0000036$

$-1.1648636$

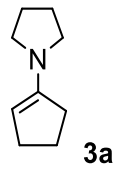
2. 9387730 3. 0081640 1.5559860 0.7309950 $-0.6383760$

$-1.3870310$

$-1.4902950$

$-2.8834110$

$-2.8352040$

3.0537050

3. 7221880

3. 6176980

3.4735090

$-1.2660410$

$-1.0281650$

$-1.5041920$

$-1.1328700$

$-3.6886020$

$-3.0313430$

$-3.0287450$

$-3.5599950$

1. 5029710

1.1114210

1.2396240

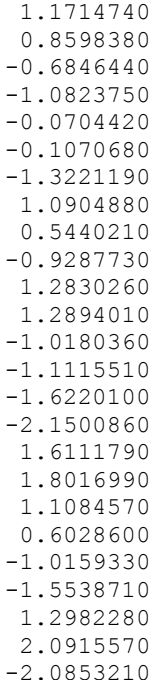

$-0.4372510$

0.0851120

0.1732070

0.2895500

$-0.0591280$

$-0.1973320$

0.1007340

$-0.1300090$

0.2369310

$-0.2009310$

1.0871660

$-0.5435420$

1.0200310

$-0.7279000$

1.1553820

$-0.5184960$

$-1.0955410$

0.6225110

$-0.2373470$

1. 3193700

$-1.2743470$

0.3243400

$-1.5283730$

$-0.0093220$

0.5451840 
Table 2. Calcd vs. Exptl $\Delta G^{\circ}$ Values for the Formation of Enamine 5a from 5

\begin{tabular}{|c|c|c|c|c|c|c|}
\hline & & $\mathrm{H}$ & a & $5 a$ & $\mathrm{H}_{2} \mathrm{O}$ & $\begin{array}{c}\text { reaction } \\
\text { energy } \\
(\mathrm{kcal} / \mathrm{mol})\end{array}$ \\
\hline B3LYP/6-31G(d) & $\begin{array}{l}\mathrm{E} \\
\mathrm{G}^{\circ} \\
\left.\mathrm{G}^{\circ} \mathrm{DMSO}\right)\end{array}$ & $\begin{array}{l}-271.77284 \\
-271.66215 \\
-271.66879\end{array}$ & $\begin{array}{l}-212.58173 \\
-212.47948 \\
-212.48462\end{array}$ & $\begin{array}{l}-407.92970 \\
-407.71956 \\
-407.72609\end{array}$ & $\begin{array}{l}-76.40895 \\
-76.40546 \\
-76.41304\end{array}$ & $\begin{array}{r}10.0 \\
10.4 \\
9.0\end{array}$ \\
\hline$B 3 L Y P / 6-31+G(d)$ & $\begin{array}{l}\mathrm{E} \\
\mathrm{G}^{\circ} \\
\left.\mathrm{o}^{\circ} \mathrm{DMSO}\right)\end{array}$ & $\begin{array}{l}-271.78411 \\
-271.67398 \\
-271.68242\end{array}$ & $\begin{array}{l}-212.59104 \\
-212.48910 \\
-212.49551\end{array}$ & $\begin{array}{l}-407.94317 \\
-407.73381 \\
-407.74108\end{array}$ & $\begin{array}{l}-76.42257 \\
-76.41914 \\
-76.42802\end{array}$ & $\begin{array}{l}5.9 \\
6.4 \\
5.5\end{array}$ \\
\hline B3LYP/6-31+G(d,p) & $\begin{array}{l}\mathrm{E} \\
\mathrm{G}^{\circ} \\
\left.{ }^{\circ} \mathrm{DMSO}\right)\end{array}$ & $\begin{array}{l}-271.79762 \\
-271.68809 \\
-271.69654\end{array}$ & $\begin{array}{l}-212.60466 \\
-212.50318 \\
-212.50947\end{array}$ & $\begin{array}{l}-407.96617 \\
-407.75776 \\
-407.76499\end{array}$ & $\begin{array}{l}-76.43405 \\
-76.43041 \\
-76.43898\end{array}$ & $\begin{array}{l}1.3 \\
1.9 \\
1.3\end{array}$ \\
\hline B3LYP/6-311+G(d,p) & $\begin{array}{l}\mathrm{E} \\
\mathrm{G}^{\circ} \\
\mathrm{G}^{\circ}(\mathrm{DMSO})\end{array}$ & $\begin{array}{l}-271.85569 \\
-271.74663 \\
-271.75499\end{array}$ & $\begin{array}{l}-212.64634 \\
-212.54526 \\
-212.55142\end{array}$ & $\begin{array}{l}-408.04270 \\
-407.83514 \\
-407.84254\end{array}$ & $\begin{array}{l}-76.45846 \\
-76.45548 \\
-76.46378\end{array}$ & $\begin{array}{l}0.5 \\
0.8 \\
0.0\end{array}$ \\
\hline M06-2X/6-31G(d) & $\begin{array}{l}\mathrm{E} \\
\mathrm{G}^{\circ} \\
\left.{ }^{\circ} \mathrm{DMSO}\right)\end{array}$ & $\begin{array}{l}-271.63489 \\
-271.52300 \\
-271.52976\end{array}$ & $\begin{array}{l}-212.47250 \\
-212.36883 \\
-212.37448\end{array}$ & $\begin{array}{l}-407.72227 \\
-407.50997 \\
-407.51709\end{array}$ & $\begin{array}{l}-76.37339 \\
-76.37018 \\
-76.37806\end{array}$ & $\begin{array}{l}7.4 \\
7.3 \\
5.7\end{array}$ \\
\hline$M 06-2 X / 6-31+G(d)$ & $\begin{array}{l}\mathrm{E} \\
\mathrm{G}^{\circ} \\
\mathrm{G}^{\circ}(\mathrm{DMSO})\end{array}$ & $\begin{array}{l}-271.64330 \\
-271.53169 \\
-271.53984\end{array}$ & $\begin{array}{l}-212.47976 \\
-212.37630 \\
-212.38308\end{array}$ & $\begin{array}{l}-407.73310 \\
-407.52130 \\
-407.52920\end{array}$ & $\begin{array}{l}-76.38375 \\
-76.38062 \\
-76.38958\end{array}$ & $\begin{array}{l}3.9 \\
3.8 \\
2.5\end{array}$ \\
\hline M06-2X/6-311+G(d,p) & $\begin{array}{l}\mathrm{E} \\
G^{\circ} \\
G^{\circ} \text { (DMSO) }\end{array}$ & $\begin{array}{l}-271.71781 \\
-271.60732 \\
-271.61564\end{array}$ & $\begin{array}{l}-212.53676 \\
-212.43419 \\
-212.44084\end{array}$ & $\begin{array}{l}-407.83625 \\
-407.62624 \\
-407.63455\end{array}$ & $\begin{array}{l}-76.42083 \\
-76.41747 \\
-76.42581\end{array}$ & $\begin{array}{l}-1.6 \\
-1.4 \\
-2.3\end{array}$ \\
\hline $\begin{array}{l}\text { MP2/6-31G(d) } \\
\text { //B3LYP/6-31G(d) }\end{array}$ & $\begin{array}{l}\mathrm{E}^{\circ} \\
\mathrm{G}^{\circ} \text { (DMSO) }\end{array}$ & $\begin{array}{l}-270.84726 \\
-270.73657 \\
-270.74295\end{array}$ & $\begin{array}{l}-211.82693 \\
-211.72468 \\
-211.73049\end{array}$ & $\begin{array}{l}-406.46700 \\
-406.25687 \\
-406.26385\end{array}$ & $\begin{array}{l}-76.19684 \\
-76.19335 \\
-76.20154\end{array}$ & $\begin{array}{l}6.5 \\
6.9 \\
5.1\end{array}$ \\
\hline $\begin{array}{l}\text { MP2/6-31+G(d) } \\
\text { //B3LYP/6-31G(d) }\end{array}$ & $\begin{array}{l}\mathrm{E} \\
\mathrm{G}^{\circ} \\
\left.{ }^{\circ} \mathrm{DMSO}\right)\end{array}$ & $\begin{array}{l}-270.86214 \\
-270.75144 \\
-270.75932\end{array}$ & $\begin{array}{l}-211.83986 \\
-211.73761 \\
-211.74479\end{array}$ & $\begin{array}{l}-406.48907 \\
-406.27893 \\
-406.28671\end{array}$ & $\begin{array}{l}-76.20967 \\
-76.20618 \\
-76.21559\end{array}$ & $\begin{array}{l}2.0 \\
2.5 \\
1.1\end{array}$ \\
\hline $\begin{array}{l}\text { MP2/6-311+G(d,p) } \\
\text { //B3LYP/6-311+G(d,p) }\end{array}$ & $\begin{array}{l}\mathrm{E} \\
G^{\circ} \\
G^{\circ}(\mathrm{DMSO})\end{array}$ & $\begin{array}{l}-271.03891 \\
-270.92985 \\
-270.93720\end{array}$ & $\begin{array}{l}-211.98191 \\
-211.88083 \\
-211.88729\end{array}$ & $\begin{array}{l}-406.75141 \\
-406.54385 \\
-406.55129\end{array}$ & $\begin{array}{l}-76.27464 \\
-76.27165 \\
-76.28001\end{array}$ & $\begin{array}{l}-3.3 \\
-3.0 \\
-3.5\end{array}$ \\
\hline $\begin{array}{l}\text { MP2/6-311+G(d,p) } \\
\text { //M06-2X/6-311+G(d,p) }\end{array}$ & $\begin{array}{l}\mathrm{E}^{\circ} \\
G^{\circ}(\mathrm{DMSO})\end{array}$ & $\begin{array}{l}-271.03890 \\
-270.92841 \\
-270.93562\end{array}$ & $\begin{array}{l}-211.98209 \\
-211.87952 \\
-211.88601\end{array}$ & $\begin{array}{l}-406.75194 \\
-406.54193 \\
-406.54935\end{array}$ & $\begin{array}{l}-76.27465 \\
-76.27129 \\
-76.27961\end{array}$ & $\begin{array}{l}-3.5 \\
-3.3 \\
-4.6\end{array}$ \\
\hline $\begin{array}{l}\operatorname{CCSD}(T) / 6-31+G(d) \\
/ / B 3 L Y P / 6-31+G(d)\end{array}$ & $\begin{array}{l}\mathrm{E}^{\circ} \\
\mathbf{G}^{\circ} \text { (DMSO) }\end{array}$ & $\begin{array}{l}-270.92020 \\
-270.81007 \\
-270.81796\end{array}$ & $\begin{array}{l}-211.89247 \\
-211.79047 \\
-211.79737\end{array}$ & $\begin{array}{l}-406.59936 \\
-406.38806 \\
-406.39547\end{array}$ & $\begin{array}{l}-76.21783 \\
-76.21440 \\
-76.22350\end{array}$ & $\begin{array}{l}-2.8 \\
-2.4 \\
-3.2\end{array}$ \\
\hline \multirow[t]{2}{*}{$\begin{array}{l}\operatorname{CCSD}(T) / 6-311+G(d, p) \\
/ / B 3 L Y P / 6-311+G(d, p)\end{array}$} & \multirow[t]{2}{*}{$\begin{array}{l}\mathbf{E}^{\circ} \\
\mathbf{G}^{\circ}(\mathrm{DMSO})\end{array}$} & \multirow[t]{2}{*}{$\begin{array}{l}-271.09792 \\
-270.98887 \\
-270.99645\end{array}$} & $\begin{array}{l}-212.03512 \\
-211.93404 \\
-211.94023\end{array}$ & $\begin{array}{l}-406.85700 \\
-406.64937 \\
-406.65609\end{array}$ & $\begin{array}{l}-76.28099 \\
-76.27800 \\
-76.28617\end{array}$ & $\begin{array}{l}-3.1 \\
-2.8 \\
-3.5\end{array}$ \\
\hline & & & \multicolumn{2}{|c|}{$\begin{array}{l}K_{\text {eq }}=14.3\left(\mathrm{C}_{6} \mathrm{D}_{6}\right) \\
K_{\text {eq }} \geq 500\left(\mathrm{DMSO}-d_{6}\right)\end{array}$} & $\begin{aligned} \mathrm{ptl} & =-1 \\
\mathrm{ptl} & \leq-3\end{aligned}$ & $\begin{array}{l}\mathrm{al} / \mathrm{mol} \\
\mathrm{al} / \mathrm{mol}\end{array}$ \\
\hline
\end{tabular}

- All the calculations of pyrrolidine were carried out on the conformation with equatorial $\mathrm{NH}(0.1-0.3 \mathrm{kcal} / \mathrm{mol}$ more stable, in the gas phase). Only at the B3LYP/6-31G(d) level, the conformation with the axial NH proton is predicted to have a slightly lower energy $(\Delta \mathrm{E}=-0.15 \mathrm{kcal} / \mathrm{mol})$.

- It is observed that single-point MP2/6-311+G(d,p) calculations gave practically identical energies from B3LYP/6-311+G(d,p) and M06-2X/6$311+G(d, p)$ geometries. This is quite general (preceding and following Tables).

Differences among continuum solvation models, at the B3LYP/6-31G(d) level (E in DMSO)

$\begin{array}{lcccccc} & \mathbf{5} & \mathbf{a} & \mathbf{5 a} & + & \mathrm{H}_{2} \mathrm{O} & \Delta \mathrm{E}(\mathrm{kcal} / \mathrm{mol}) \\ \text { SMD } & -271.79227 & -212.59735 & -407.95027 & -76.43146 & 5.0 \\ \text { CPCM } & -271.79009 & -212.59476 & -407.94606 & -76.43120 & 4.8 \\ \text { IEFPCM } & -271.79002 & -212.59469 & -407.94600 & -76.43111 & 4.8\end{array}$




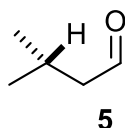

5
0.1008981 $-0.3852739$ 0.6957961

$-1.4242159$

0.2509551

$-0.2760709$

0.2170281

1. 2974551

$-0.3855889$

$-0.9036629$

0.6359491

$-0.8857999$

$-0.6573279$

0.3194521

1.4052691

$-0.0048619$

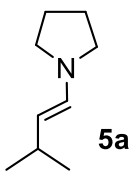

$-0.1272154$

$-0.3370854$

0.3471146

0.1438886

$-0.6690864$

$-0.3290914$

1.0595106

$-1.2047814$

$-0.4771744$

1.0048126

$-0.4853634$

$-0.6563044$

0.9359506

$-1.4153534$

0.1556576

$-0.0093564$

1. 4323266

1. 2068776

$-1.7313214$

1.6955026

1.4559836

$-2.2054254$

$-1.3072544$

$-0.6946704$

$-0.7672814$

1. 6736176

1. 3055216
$-1.2648997$

0.0528133

1. 2198573

$-0.0579727$

0.0302583

$-0.1153717$

$-0.2239147$

0.3310123

$-0.1688617$

0.0298663

$-1.1828827$

$-2.0906407$

$-1.5268127$

0.2577203

2.1698573

1.3012763

1.0730083

$-0.2442157$

0.2080763

0.5133973

$-1.2171817$

$-0.0957177$

1.4292663

$-1.2298397$

0.3721123

$-0.6293407$

1.0591303

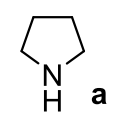

$$
\begin{array}{r}
-0.0010159 \\
-0.8934569 \\
0.4652881 \\
0.7921461 \\
-0.1181529 \\
-1.8671389 \\
-1.0850979 \\
1.3312961 \\
-0.3150369 \\
1.8445131 \\
0.6073651 \\
0.4882481 \\
-0.7948539 \\
-0.4541029
\end{array}
$$

0.4421729

0.9053649

$-0.8779711$

$-0.6526721$

0.5370909

0.3845559

1.9771579

$-1.1971071$

$-1.6532061$

$-0.3895341$

$-1.5518811$

1. 3814889

0.2762699

0.4182699
$-1.2499449$

$-0.1828459$

$-0.8146799$

0.6751541

1.0983131

$-0.1929349$

$-0.2738679$

$-1.3994189$

$-0.9124729$

0.7973821

1.2663101

1.4312841

1. 9145391

$-2.1568179$ 


\begin{tabular}{|c|c|c|c|c|c|c|}
\hline & & 4 & $\stackrel{\mathrm{N}}{\mathrm{H}}$ a & $4 a$ & $\mathrm{H}_{2} \mathrm{O}$ & $\begin{array}{c}\text { reaction } \\
\text { energy } \\
(\mathrm{kcal} / \mathrm{mol})\end{array}$ \\
\hline B3LYP/6-31G(d) & $\begin{array}{l}\mathrm{E} \\
G^{\circ} \\
G^{\circ} \text { (DMSO) }\end{array}$ & $\begin{array}{l}-193.14535 \\
-193.08774 \\
-193.09433\end{array}$ & $\begin{array}{l}-212.58173 \\
-212.47948 \\
-212.48462\end{array}$ & $\begin{array}{l}-329.30152 \\
-329.14399 \\
-329.14999\end{array}$ & $\begin{array}{l}-76.40895 \\
-76.40546 \\
-76.41304\end{array}$ & $\begin{array}{r}10.4 \\
10.8 \\
9.6\end{array}$ \\
\hline B3LYP/6-31+G(d) & $\begin{array}{l}\mathrm{E} \\
G^{\circ} \\
G^{\circ} \text { (DMSO) }\end{array}$ & $\begin{array}{l}-193.15517 \\
-193.09789 \\
-193.10633\end{array}$ & $\begin{array}{l}-212.59097 \\
-212.48787 \\
-212.49426\end{array}$ & $\begin{array}{l}-329.31291 \\
-329.15578 \\
-329.16255\end{array}$ & $\begin{array}{l}-76.42257 \\
-76.41914 \\
-76.42802\end{array}$ & $\begin{array}{l}6.7 \\
7.2 \\
6.7\end{array}$ \\
\hline B3LYP/6-311+G(d,p) & $\begin{array}{l}\mathrm{E} \\
G^{\circ} \\
G^{\circ} \text { (DMSO) }\end{array}$ & $\begin{array}{l}-193.20735 \\
-193.15076 \\
-193.15908\end{array}$ & $\begin{array}{l}-212.64634 \\
-212.54526 \\
-212.55142\end{array}$ & $\begin{array}{l}-329.39325 \\
-329.23763 \\
-329.24444\end{array}$ & $\begin{array}{l}-76.45846 \\
-76.45548 \\
-76.46378\end{array}$ & $\begin{array}{l}1.2 \\
1.8 \\
1.4\end{array}$ \\
\hline M06-2X/6-31G(d) & $\begin{array}{l}\mathrm{E} \\
\mathrm{G}^{\circ} \\
\mathrm{G}^{\circ} \text { (DMSO) }\end{array}$ & $\begin{array}{l}-193.04739 \\
-192.98862 \\
-192.99532\end{array}$ & $\begin{array}{l}-212.47250 \\
-212.36883 \\
-212.37448\end{array}$ & $\begin{array}{l}-329.13509 \\
-328.97549 \\
-328.98204\end{array}$ & $\begin{array}{l}-76.37339 \\
-76.37018 \\
-76.37806\end{array}$ & $\begin{array}{l}7.2 \\
7.4 \\
6.1\end{array}$ \\
\hline$M 06-2 X / 6-31+G(d)$ & $\begin{array}{l}E \\
G^{\circ} \\
G^{\circ}(D M S O)\end{array}$ & $\begin{array}{l}-193.05468 \\
-192.99637 \\
-193.00453\end{array}$ & $\begin{array}{l}-212.47978 \\
-212.37631 \\
-212.38310\end{array}$ & $\begin{array}{l}-329.14411 \\
-328.98474 \\
-328.99202\end{array}$ & $\begin{array}{l}-76.38376 \\
-76.38062 \\
-76.38958\end{array}$ & $\begin{array}{l}4.1 \\
4.6 \\
3.8\end{array}$ \\
\hline$M 06-2 X / 6-311+G(d, p)$ & $\begin{array}{l}\mathrm{E} \\
G^{\circ} \\
G^{\circ} \text { (DMSO) }\end{array}$ & $\begin{array}{l}-193.10901 \\
-193.05140 \\
-193.05965\end{array}$ & $\begin{array}{l}-212.53676 \\
-212.43419 \\
-212.44084\end{array}$ & $\begin{array}{l}-329.22734 \\
-329.06965 \\
-329.07722\end{array}$ & $\begin{array}{l}-76.42083 \\
-76.41747 \\
-76.42581\end{array}$ & $\begin{array}{l}-1.5 \\
-1.0 \\
-1.6\end{array}$ \\
\hline $\begin{array}{l}\text { MP2/6-31+G(d) } \\
\text { //B3LYP/6-31G(d) }\end{array}$ & $\begin{array}{l}\mathrm{E}^{\circ} \\
\mathrm{G}^{\circ} \text { (DMSO) }\end{array}$ & $\begin{array}{l}-192.52532 \\
-192.46771 \\
-192.47555\end{array}$ & $\begin{array}{l}-211.83986 \\
-211.73761 \\
-211.74479\end{array}$ & $\begin{array}{l}-328.14994 \\
-327.99241 \\
-327.99956\end{array}$ & $\begin{array}{l}-76.20967 \\
-76.20618 \\
-76.21559\end{array}$ & $\begin{array}{l}3.5 \\
3.9 \\
2.9\end{array}$ \\
\hline $\begin{array}{l}\text { MP2/6-311+G(d,p) } \\
\text { //B3LYP/6-311+G(d,p) }\end{array}$ & $\begin{array}{l}\mathrm{E}^{\circ} \\
\mathrm{G}^{\circ} \\
\mathrm{G}^{\circ} \text { (DMSO) }\end{array}$ & $\begin{array}{l}-192.64531 \\
-192.58871 \\
-192.59598\end{array}$ & $\begin{array}{l}-211.98191 \\
-211.88083 \\
-211.88729\end{array}$ & $\begin{array}{l}-328.35574 \\
-328.20011 \\
-328.20691\end{array}$ & $\begin{array}{l}-76.27464 \\
-76.27165 \\
-76.28001\end{array}$ & $\begin{array}{l}-2.0 \\
-1.4 \\
-2.3\end{array}$ \\
\hline $\begin{array}{l}\text { MP2/6-311+G(d,p) } \\
\text { //M06-2X/6-311+G(d,p) }\end{array}$ & $\begin{array}{l}\mathrm{E}^{\circ} \\
\mathrm{G}^{\circ} \\
\left.{ }^{\circ} \mathrm{DMSO}\right)\end{array}$ & $\begin{array}{l}-192.64514 \\
-192.58753 \\
-192.59463\end{array}$ & $\begin{array}{l}-211.98209 \\
-211.87952 \\
-211.88601\end{array}$ & $\begin{array}{l}-328.35615 \\
-328.19846 \\
-328.20523\end{array}$ & $\begin{array}{l}-76.27465 \\
-76.27129 \\
-76.27961\end{array}$ & $\begin{array}{l}-2.2 \\
-1.7 \\
-2.6\end{array}$ \\
\hline
\end{tabular}

Cartesian coordinates of the stationary points, at the B3LYP/6-311+G(d,p) level
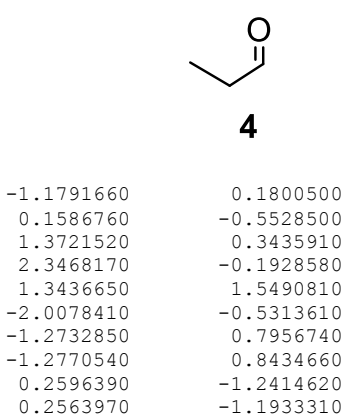

$-0.0071150$

0.0157130

$-0.0070530$

0.0103930

$-0.0415710$

0.0104720

0.9038890

0.8544890

$-0.8350810$

$-0.8036420$
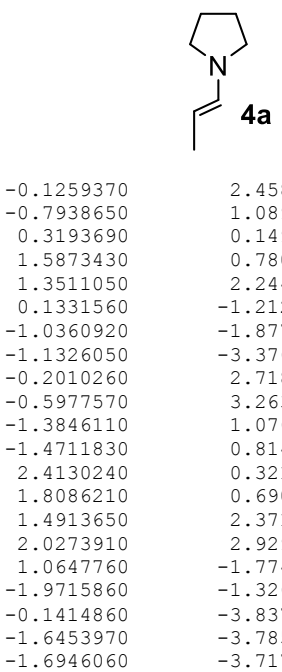

2.4588140
1.0899720
0.1499770
0.7807520
2.2440020
-1.2123570
-1.8774760
-3.3765320
2.7180550
3.2634670
1.0763060
0.8145190
0.3210260
0.6900140
2.3719880
2.9292020
-1.7740300
-1.3267200
-3.8376220
-3.7857460
-3.7176120

0.1657179 $-0.0883681$ $-0.1909151$ 0.1639569 $-0.2159591$ $-0.0868431$ $-0.0521601$ 0.0007759 1.2255859 $-0.4010211$ $-1.0126651$ $-0.7326269$ $-0.3867621$ $-.3867621$ 1.2411719 $-1.2931711$ 0.2989879 $-0.0621481$ 0.0847721 0.0460269 0.8784871 
Table 3. Calcd vs. Exptl $\Delta G^{\circ}$ Values for the Formation of Enamine $5 \mathrm{~b}$ from 5

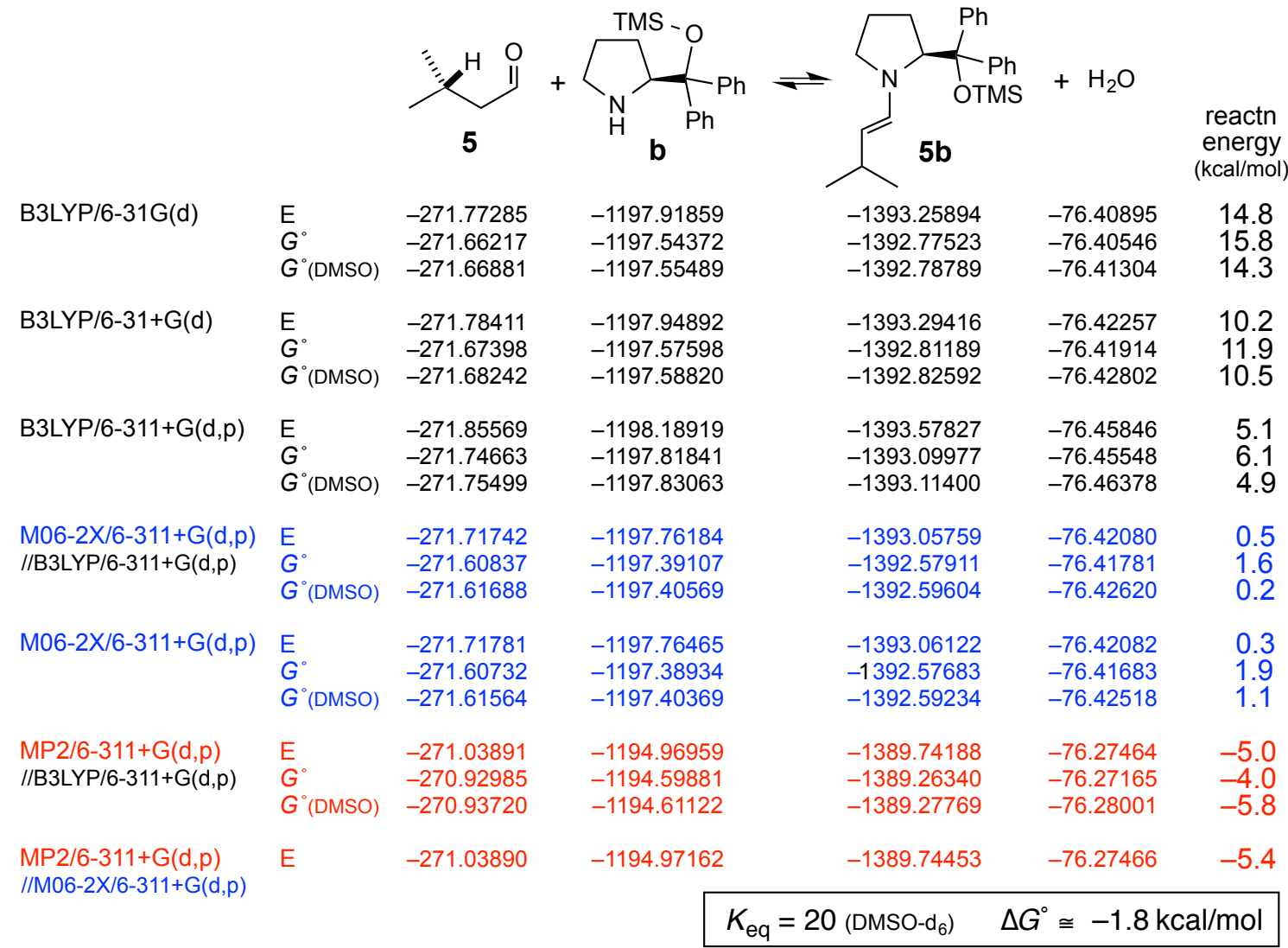

- The lowest-energy conformers are indicated [based on MMFF94s and B3LYP/6-31G(d), and confirmed at higher levels, see next page]. Also see ref 17 of the main text. In the case of $\mathbf{b}$ (the $\mathrm{J}-\mathrm{H}$ catalyst), proton $\mathrm{NH}$ and the $\mathrm{CPh}_{2}(\mathrm{OTMS})$ group are equatorial. The lowest-energy conformer of enamine $5 \mathbf{b}$ has an envelope ring conformation ( $\mathrm{N}$ down), with the alkenyl group almost equatorial and $\mathrm{CPh}_{2}(\mathrm{OTMS})$ almost axial. However, the energy differences among the main conformers are small and do not affect the discussion and the essential conclusions.

- $\quad M 06-2 X / 6-311+G(d, p)$ (with geometry optimization at the same level) gave almost identical result (total electronic energy) as the single-point M06-2X/6-311+G(d,p)//B3LYP/6-311+G(d,p) calculation. Single-point MP2/6-311+G(d,p) calculations on B3LYP/6-311+G(d,p) and M06-2X/6$311+\mathrm{G}(\mathrm{d}, \mathrm{p})$ geometries also gave similar energies. In fact, the geometries are almost identical.

Cartesian coordinates of stationary points (minimum energy conformers), at the B3LYP/6-311+G(d,p) level
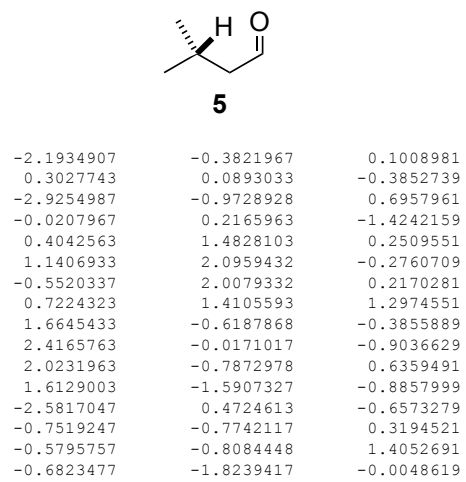<smiles>COC(c1ccccc1)(c1ccccc1)C1NCCO1</smiles>

$-3.0817560$ $-3.2443890$ $-0.5127050$ -0.2626200
-2.1325540 $-2.1325540$ .3484690 1.6833240
0.4279660 0.4279660
2.1553780 2.4353110 3.3393400
4.0808220 3.0808220
3.6250150 -0.2034420
-0.0604200 0.7259970 0.7259970
1.3751940 1.2287000 0.2179160 $-0.8407710$ $-2.0013650$ 1.6091400 .6871990 .005699 .1955610 $-0.8100910$ 0.5664860
0.8377630 2.0008210 0.9299220 0.9898850 0.98988850
-0.2537810

$\begin{array}{rr}0.3563973 & -1.7421874 \\ -0.3742217 & -2.5520294 \\ 1.4017553 & -1.9990074 \\ 0.0100473 & -1.3331714 \\ 2.1623363 & -1.3103524 \\ 1.4434213 & -2.8964844 \\ -0.1363087 & -0.736584 \\ -1.0894317 & -0.4535844 \\ -0.1526297 & -0.2975184 \\ 0.1363813 & -1.0217834 \\ -1.5766067 & 0.2992746 \\ -0.729427 & -2.0157064 \\ 1.2725933 & -0.7172014 \\ -0.4589407 & -2.6967774 \\ 0.6812313 & -2.3893084 \\ 1.5420403 & -1.394644 \\ -2.6909717 & -0.2604074 \\ -3.9601897 & 0.3061596 \\ -4.1386007 & 1.4388586 \\ -3.0369307 & 1.9979666 \\ -1.7754737 & 1.4320526 \\ 0.7947473 & 0.7469186 \\ 1.2148733 & 1.9864766 \\ 1.898073 & 3.3098666 \\ -0.2173897 & 2.7129436 \\ 2.5930103 & 1.4441276 \\ -1.6360937 & -2.2517784 \\ 1.9401673 & 0.0570066 \\ -1.1446967 & -3.4616584 \\ 0.8906713 & -2.9150704 \\ 2.4290453 & -1.1408554 \\ -2.5921097 & -1.1526444 \\ -4.8069777 & -0.1445624 \\ -5.1229617 & 1.8791346 \\ -3.1628607 & 2.8749116 \\ -0.9267757 & 1.8668166 \\ 2.7137693 & 2.916186 \\ 1.1272413 & 3.6918216 \\ 2.2930733 & 4.1585116 \\ & \end{array}$



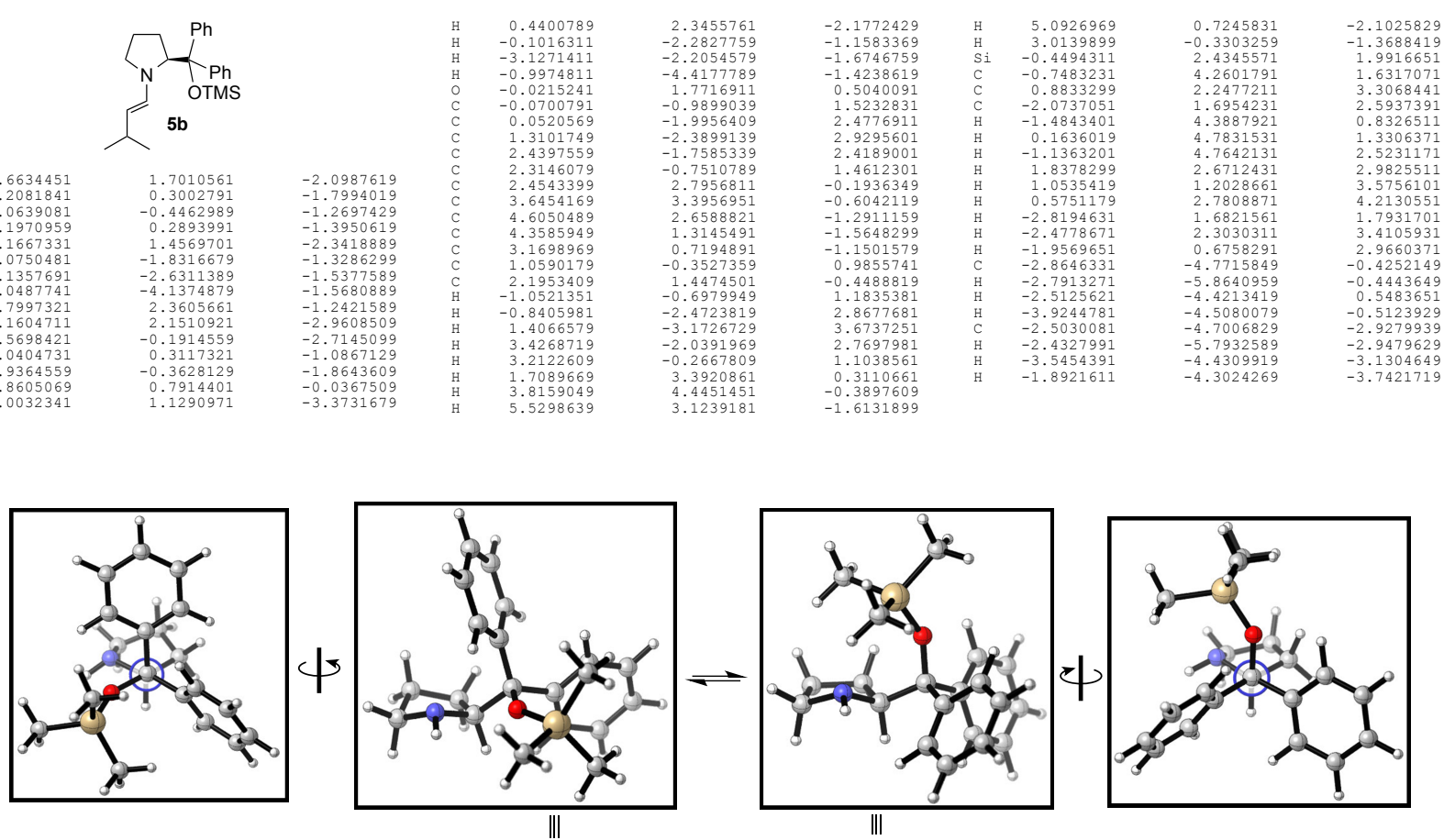

B3LYP/6-31G(d)

$B 3 L Y P / 6-31+G(d)$
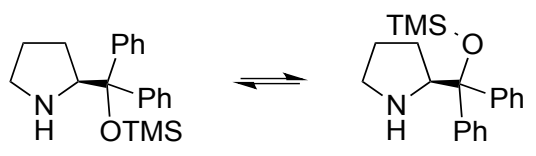

$B 3 L Y P / 6-311+G(d, p)$

$-1197.91342$

$-1197.91859$

$\Delta \mathrm{E}$

M06-2X/6-311+G(d,p)//B3LYP/6-311+G(d,p)

$-1197.94455$

$-1197.94892$

$-3.2$

$-1198.18458$

$-1198.18919$

$-2.7$

$-1197.75530$

$-1197.76184$

$-2.9$

MP2/6-311+G(d,p)//B3LYP/6-311+G(d,p)
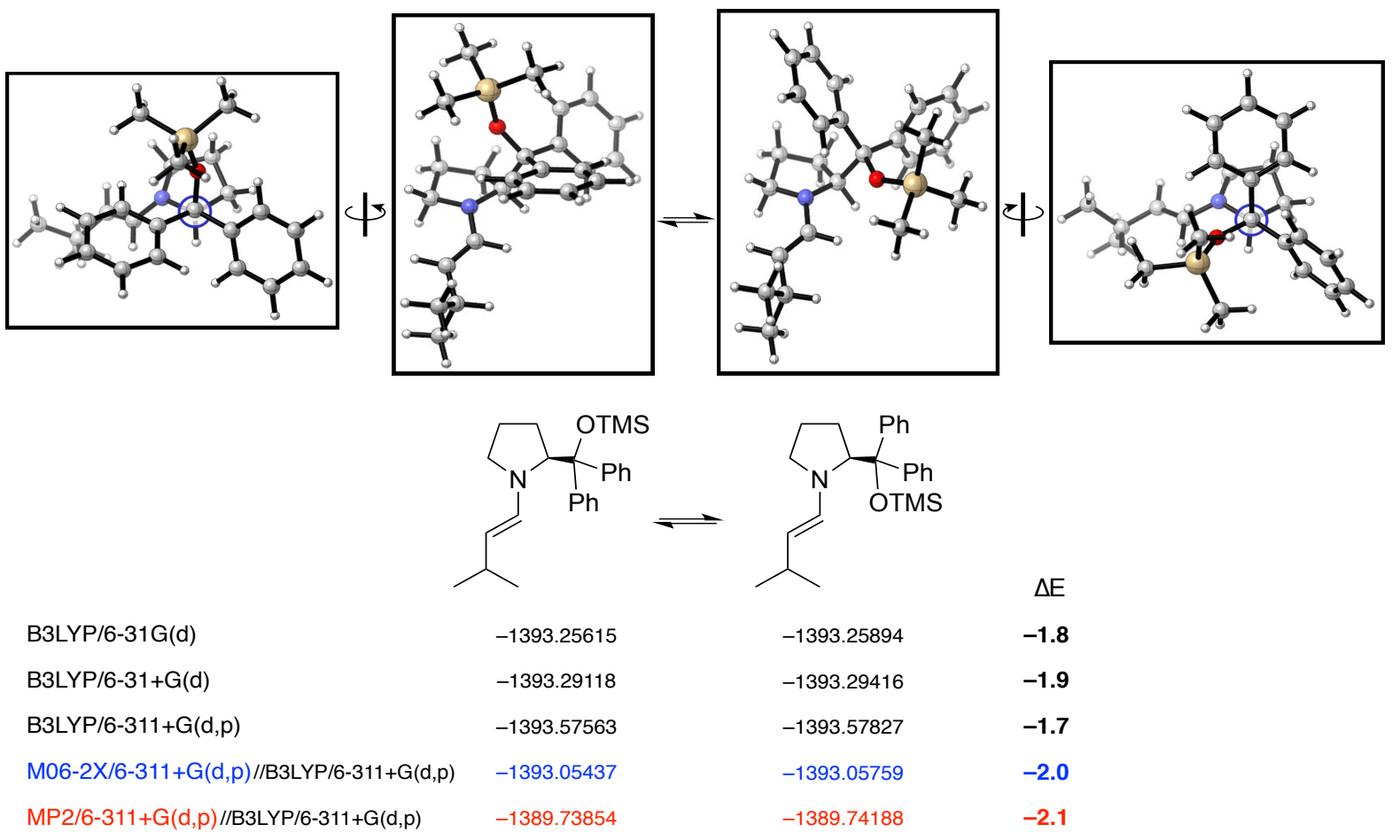

- Conformers of lower energy were not found. 
Table 4. Calcd $\Delta G^{\circ}$ Values for the Formation of Hemiaminal HA-4a

\begin{tabular}{|c|c|c|c|c|c|}
\hline & & 4 & $\mathrm{H}$ a & HA-4a & $\begin{array}{l}\text { reaction } \\
\text { energy } \\
(\mathrm{kcal} / \mathrm{mol})\end{array}$ \\
\hline B3LYP/6-31G(d) & $\begin{array}{l}\mathrm{E} \\
G^{\circ} \\
G^{\circ}(\mathrm{DMSO})\end{array}$ & $\begin{array}{l}-193.14534 \\
-193.08777 \\
-193.09438\end{array}$ & $\begin{array}{l}-212.58173 \\
-212.47948 \\
-212.48462\end{array}$ & $\begin{array}{l}-405.73808 \\
-405.55352 \\
-405.56056\end{array}$ & $\begin{array}{r}-6.9 \\
8.7 \\
11.6\end{array}$ \\
\hline$B 3 L Y P / 6-31+G(d)$ & $\begin{array}{l}\mathrm{E} \\
G^{\circ} \\
G^{\circ}(\mathrm{DMSO})\end{array}$ & $\begin{array}{l}-193.15517 \\
-193.09789 \\
-193.10633\end{array}$ & $\begin{array}{l}-212.59104 \\
-212.48910 \\
-212.49551\end{array}$ & $\begin{array}{l}-405.75530 \\
-405.57125 \\
-405.57993\end{array}$ & $\begin{array}{r}-5.7 \\
9.9 \\
13.7\end{array}$ \\
\hline B3LYP/6-311+G(d,p) & $\begin{array}{l}\mathrm{E} \\
G^{\circ} \\
G^{\circ}(\mathrm{DMSO})\end{array}$ & $\begin{array}{l}-193.20734 \\
-193.15076 \\
-193.15909\end{array}$ & $\begin{array}{l}-212.64634 \\
-212.54526 \\
-212.55142\end{array}$ & $\begin{array}{l}-405.86311 \\
-405.68043 \\
-405.68896\end{array}$ & $\begin{array}{r}-5.9 \\
9.8 \\
13.6\end{array}$ \\
\hline $\begin{array}{l}\text { B2PLYP-D3/6-311+G(d,p) } \\
\text { //B3LYP/6-31+G(d) }\end{array}$ & $\begin{array}{l}\mathrm{E} \\
G^{\circ} \\
G^{\circ}(\mathrm{DMSO})\end{array}$ & $\begin{array}{l}-192.80017 \\
-192.74289 \\
-192.75198\end{array}$ & $\begin{array}{l}-212.15716 \\
-212.05521 \\
-212.06156\end{array}$ & $\begin{array}{l}-404.97259 \\
-404.78854 \\
-405.79742\end{array}$ & $\begin{array}{r}-9.6 \\
6.0 \\
10.1\end{array}$ \\
\hline $\begin{array}{l}\text { wB97XD/6-311+G(d,p) } \\
\text { //B3LYP/6-31+G(d) }\end{array}$ & $\begin{array}{l}\mathrm{E} \\
G^{\circ} \\
G^{\circ}(\mathrm{DMSO})\end{array}$ & $\begin{array}{l}-193.13820 \\
-193.08092 \\
-193.08949\end{array}$ & $\begin{array}{l}-212.57692 \\
-212.47498 \\
-212.48154\end{array}$ & $\begin{array}{l}-405.73824 \\
-405.55419 \\
-405.56316\end{array}$ & $\begin{array}{r}-14.5 \\
1.1 \\
4.9\end{array}$ \\
\hline M06-2X/6-31G(d) & $\begin{array}{l}\mathrm{E} \\
G^{\circ} \\
G^{\circ}(\mathrm{DMSO})\end{array}$ & $\begin{array}{l}-193.04740 \\
-192.98866 \\
-192.99536\end{array}$ & $\begin{array}{l}-212.47250 \\
-212.36883 \\
-212.37448\end{array}$ & $\begin{array}{l}-405.54672 \\
-405.35941 \\
-405.36680\end{array}$ & $\begin{array}{r}-16.8 \\
-1.2 \\
1.9\end{array}$ \\
\hline $\begin{array}{l}\text { M06-2X/6-31+G(d) } \\
\text { //B3LYP/6-31+G(d) }\end{array}$ & $\begin{array}{l}\mathrm{E} \\
G^{\circ} \\
G^{\circ}(\mathrm{DMSO})\end{array}$ & $\begin{array}{l}-193.05443 \\
-192.99715 \\
-193.00554\end{array}$ & $\begin{array}{l}-212.47959 \\
-212.37764 \\
-212.38444\end{array}$ & $\begin{array}{l}-405.55958 \\
-405.37553 \\
-405.38453\end{array}$ & $\begin{array}{r}-16.0 \\
-0.5 \\
3.4\end{array}$ \\
\hline$M 06-2 X / 6-311+G(d, p)$ & $\begin{array}{l}\mathrm{E} \\
G^{\circ} \\
G^{\circ}(\mathrm{DMSO})\end{array}$ & $\begin{array}{l}-193.10902 \\
-193.05142 \\
-193.05966\end{array}$ & $\begin{array}{l}-212.53678 \\
-212.43401 \\
-212.44063\end{array}$ & $\begin{array}{l}-405.67255 \\
-405.48687 \\
-405.49576\end{array}$ & $\begin{array}{r}-16.8 \\
-0.9 \\
2.8\end{array}$ \\
\hline $\begin{array}{l}\text { MP2/6-311+G(d) } \\
\text { //B3LYP/6-31+G(d) }\end{array}$ & $\begin{array}{l}\mathrm{E} \\
G^{\circ} \\
G^{\circ}(\mathrm{DMSO})\end{array}$ & $\begin{array}{l}-192.64539 \\
-192.58811 \\
-192.59545\end{array}$ & $\begin{array}{l}-211.98188 \\
-211.87994 \\
-211.88643\end{array}$ & $\begin{array}{l}-404.65210 \\
-404.46805 \\
-404.47690\end{array}$ & $\begin{array}{r}-15.6 \\
0.0 \\
3.1\end{array}$ \\
\hline $\begin{array}{l}\text { MP2/6-311+G(d) } \\
\text { //B3LYP/6-311+G(d,p) }\end{array}$ & $\begin{array}{l}\mathrm{E} \\
\mathrm{G}^{\circ} \\
\mathrm{G}^{\circ}(\mathrm{DMSO})\end{array}$ & $\begin{array}{l}-192.64531 \\
-192.58872 \\
-192.59598\end{array}$ & $\begin{array}{l}-211.98191 \\
-211.88090 \\
-211.88736\end{array}$ & $\begin{array}{l}-404.65220 \\
-404.46953 \\
-404.47831\end{array}$ & $\begin{array}{r}-15.7 \\
0.1 \\
3.2\end{array}$ \\
\hline $\begin{array}{l}\operatorname{CCSD}(\mathrm{T}) / 6-31+G(\mathbf{d}) \\
/ / B 3 L Y P / 6-31+G(\mathrm{~d})\end{array}$ & $\begin{array}{l}\mathrm{E} \\
\mathbf{G}^{\circ} \\
{ }^{\circ}(\mathrm{DMSO})\end{array}$ & $\begin{array}{l}-192.56096 \\
-192.50368 \\
-192.51195\end{array}$ & $\begin{array}{l}-211.89235 \\
-211.78925 \\
-211.79614\end{array}$ & $\begin{array}{l}-404.47421 \\
-404.28910 \\
-404.29822\end{array}$ & $\begin{array}{r}-13.0 \\
2.4 \\
6.2\end{array}$ \\
\hline $\begin{array}{l}\text { CCSD(T)/6-311+G(d,p) } \\
\text { //B3LYP/6-311+G(d,p) }\end{array}$ & $\begin{array}{l}\mathbf{E} \\
\boldsymbol{G}^{\circ} \\
\boldsymbol{G}^{\circ} \text { (DMSO) }\end{array}$ & $\begin{array}{l}-192.68131 \\
-192.62473 \\
-192.63227\end{array}$ & $\begin{array}{l}-212.03512 \\
-211.93404 \\
-211.94023\end{array}$ & $\begin{array}{l}-404.73810 \\
-404.55542 \\
-404.56393\end{array}$ & $\begin{array}{r}-13.6 \\
2.1 \\
5.4\end{array}$ \\
\hline \multicolumn{6}{|c|}{ B2PLYP-D3/6-311+G(d,p) predicts $\Delta E=-9.6 \mathrm{kcal} / \mathrm{mol}$, which represents some improvement over B3LYP/6-311+G(d,p) } \\
\hline
\end{tabular}

Cartesian coordinates of the stationary points, at the B3LYP/6-311+G(d,p) level

$\begin{array}{lrrr} & & \text { O } \\ & & & \\ & & 4 & \\ \text { C } & -1.1791660 & 0.1800500 & -0.0071150 \\ \text { C } & 0.1586760 & -0.5528500 & 0.0157130 \\ \text { C } & 1.3721520 & 0.3435910 & -0.0070530 \\ \text { H } & 2.3468170 & -0.1928580 & 0.0103930 \\ \text { O } & 1.3436650 & 1.5490810 & -0.0415710 \\ \text { H } & -2.0078410 & -0.5313610 & 0.0104720 \\ \text { H } & -1.2732850 & 0.7956740 & -0.9038890 \\ \text { H } & -1.2770540 & 0.8434660 & 0.8544890 \\ \text { H } & 0.2596390 & -1.2414620 & -0.8350810 \\ \text { H } & 0.2563970 & -1.1933310 & 0.9036420 \\ & & & \end{array}$




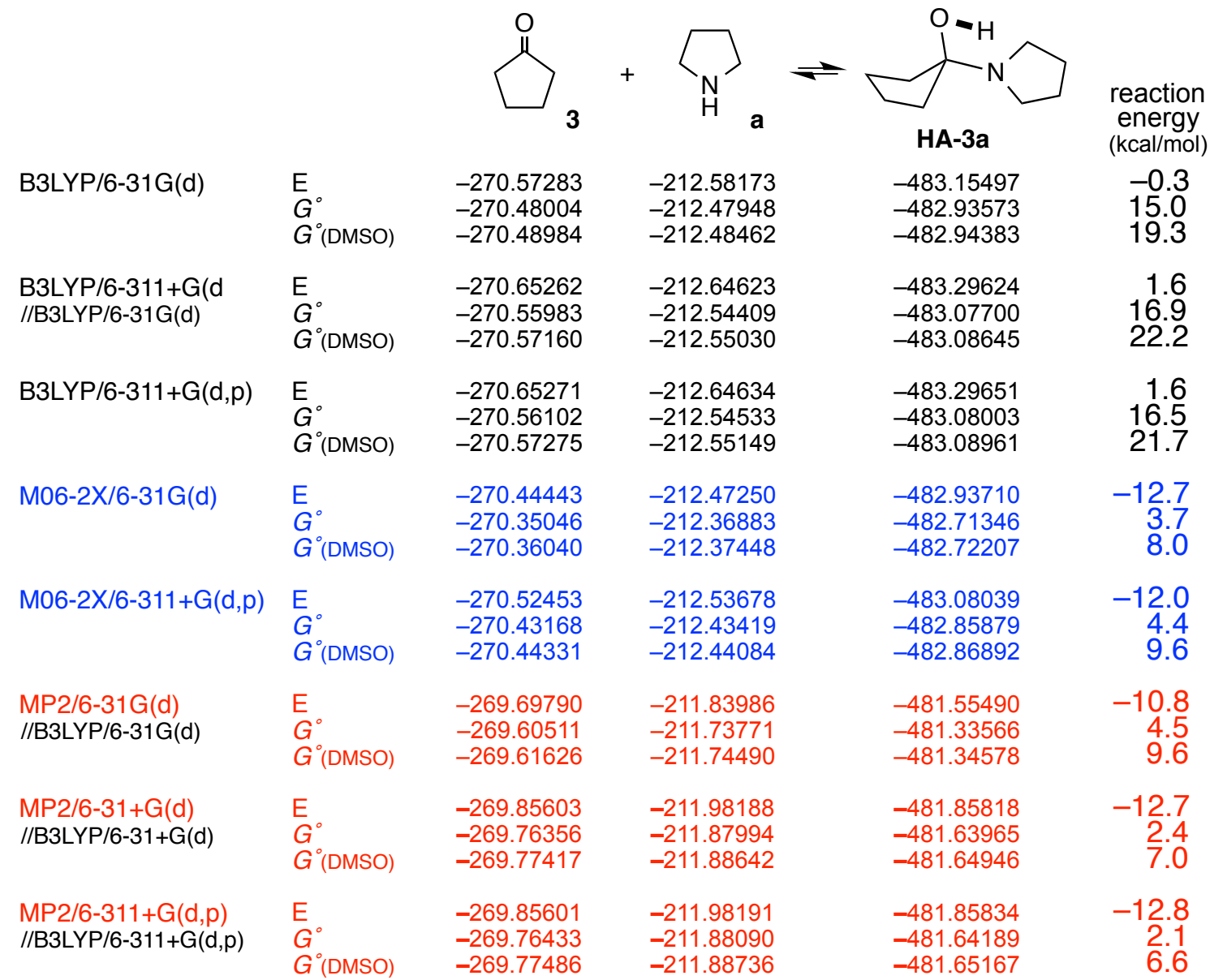

Optimized structure and Cartesian coordinates of $\boldsymbol{H A}-3 \boldsymbol{a}$, at the B3LYP/6-311+G(d,p) level

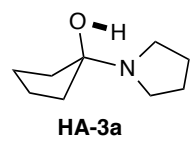

$$
\begin{array}{r}
-2.8860912 \\
-2.5322092 \\
-1.0581602 \\
-1.5469842 \\
-0.4500162 \\
0.8975808 \\
1.1715348 \\
2.5678458 \\
2.8410508 \\
1.4791718 \\
-0.3726872 \\
0.3467778 \\
-3.3135852 \\
-3.6316592 \\
-3.1734392 \\
-2.6344242 \\
-0.9791762 \\
-0.5432282 \\
-1.5559562 \\
-1.3215802 \\
0.4249108 \\
1.1549568 \\
2.5674048 \\
3.3310048 \\
3.2340398 \\
3.5640398 \\
0.8767898 \\
1.5420878
\end{array}
$$

$\begin{array}{rr}0.0811695 & 0.2295274 \\ -1.1386115 & 1.1143944 \\ -1.4108295 & 0.7918684 \\ 0.8277125 & -0.0186076 \\ 0.0051585 & 0.7094794 \\ 0.0319975 & 0.1510704 \\ -0.7362985 & -1.0702576 \\ -0.2485565 & -1.5288066 \\ 1.0304985 & -0.6957846 \\ 1.3620255 & -0.0806346 \\ 0.5627325 & 2.0301324 \\ 0.0982815 & 2.4764154 \\ -0.2527925 & -0.7205356 \\ 0.7221565 & 0.7040624 \\ -2.0011715 & 0.9184584 \\ -0.8799925 & 2.1705384 \\ -1.9142305 & -0.1763586 \\ -2.0270155 & 1.5340504 \\ 1.8483295 & 0.3649504 \\ 0.8784145 & -1.0873166 \\ -0.5359925 & -1.8585996 \\ -1.8076935 & -0.8661966 \\ -0.0400765 & -2.6008006 \\ -1.0079105 & -1.3486846 \\ 1.8522055 & -1.2980876 \\ 0.8225215 & 0.0962714 \\ 1.9541415 & -0.7917116 \\ 1.9238265 & 0.8511634\end{array}$


Addendum to Table 4-bis. Formation of Hemiaminal HA-6a from Pivalaldehyde (6)

\begin{tabular}{|c|c|c|c|c|c|}
\hline B3LYP/6-31G(d) & $\begin{array}{l}\mathrm{E} \\
G^{\circ} \\
{ }^{\circ} \text { (DMSO) }\end{array}$ & $\begin{array}{l}-271.77374 \\
-271.66337 \\
-271.66999\end{array}$ & $\begin{array}{l}-212.58173 \\
-212.47948 \\
-212.48462\end{array}$ & $\begin{array}{l}-484.35817 \\
-84.12069 \\
-84.12815\end{array}$ & $\begin{array}{l}-1.7 \\
14.0 \\
16.7\end{array}$ \\
\hline B3LYP/6-31+G(d) & $\begin{array}{l}\mathrm{E} \\
G^{\circ} \\
G^{\circ}(\mathrm{DMSO})\end{array}$ & $\begin{array}{l}-271.78551 \\
-271.67547 \\
-271.68396\end{array}$ & $\begin{array}{l}-212.59104 \\
-212.48910 \\
-212.49551\end{array}$ & $\begin{array}{l}-484.37538 \\
-484.13818 \\
-484.14724\end{array}$ & $\begin{array}{r}0.7 \\
16.6 \\
20.2\end{array}$ \\
\hline B3LYP/6-311+G(d,p) & $\begin{array}{l}\mathrm{E} \\
G^{\circ} \\
G^{\circ}(\mathrm{DMSO})\end{array}$ & $\begin{array}{l}-271.85680 \\
-271.74804 \\
-271.75640\end{array}$ & $\begin{array}{l}-212.64634 \\
-212.54533 \\
-212.55149\end{array}$ & $\begin{array}{l}-484.50262 \\
-484.26733 \\
-484.27630\end{array}$ & $\begin{array}{r}0.3 \\
16.3 \\
19.8\end{array}$ \\
\hline $\begin{array}{l}\text { B2PLYP-D3/def2-TZVP } \\
\text { //B3LYP/6-311+G(d,p) }\end{array}$ & $\begin{array}{l}\mathrm{E} \\
G^{\circ} \\
G^{\circ} \text { (DMSO) }\end{array}$ & $\begin{array}{l}-271.28971 \\
-271.17967 \\
-271.18841\end{array}$ & $\begin{array}{l}-212.17770 \\
-212.07576 \\
-212.08143\end{array}$ & $\begin{array}{l}-483.47177 \\
-483.23458 \\
-483.24335\end{array}$ & $\begin{array}{l}-2.7 \\
13.1 \\
16.6\end{array}$ \\
\hline $\begin{array}{l}\text { B2PLYP-D3/6-311+G(d,p) } \\
\text { //B3LYP/6-311+G(d,p) }\end{array}$ & $\begin{array}{l}\mathrm{E} \\
G^{\circ} \\
\text { (DMSO) }\end{array}$ & $\begin{array}{l}-271.26221 \\
-271.15344 \\
-271.16240\end{array}$ & $\begin{array}{l}-212.15752 \\
-212.05651 \\
-212.06282\end{array}$ & $\begin{array}{l}-483.42567 \\
-483.19037 \\
-483.19963\end{array}$ & $\begin{array}{l}-3.7 \\
12.3 \\
16.1\end{array}$ \\
\hline M06-2X/6-31+G(d) & $\begin{array}{l}\mathrm{E} \\
G^{\circ} \\
G^{\circ}(\mathrm{DMSO})\end{array}$ & $\begin{array}{l}-271.64661 \\
-271.53480 \\
-271.54299\end{array}$ & $\begin{array}{l}-212.47948 \\
-212.37686 \\
-212.38109\end{array}$ & $\begin{array}{l}-484.14630 \\
-483.90685 \\
-483.91625\end{array}$ & $\begin{array}{r}-12.7 \\
2.7 \\
6.0\end{array}$ \\
\hline M06-2X/6-311+G(d,p) & $\begin{array}{l}\mathrm{E} \\
G^{\circ} \\
{ }^{\circ} \text { DMSO) }\end{array}$ & $\begin{array}{l}-271.72099 \\
-271.61038 \\
-271.61872\end{array}$ & $\begin{array}{l}-212.53678 \\
-212.43419 \\
-212.44084\end{array}$ & $\begin{array}{l}-484.27882 \\
-484.04016 \\
-484.04975\end{array}$ & $\begin{array}{r}-13.2 \\
2.7 \\
6.0\end{array}$ \\
\hline $\begin{array}{l}\text { MP2/6-31+G(d) } \\
\text { //B3LYP/6-31G(d) }\end{array}$ & $\begin{array}{l}\mathrm{E} \\
G^{\circ} \\
{ }^{\circ} \text { (DMSO) }\end{array}$ & $\begin{array}{l}-270.86709 \\
-270.75672 \\
-270.76473\end{array}$ & $\begin{array}{l}-211.83986 \\
-211.73771 \\
-211.74490\end{array}$ & $\begin{array}{l}-482.72321 \\
-482.48579 \\
-482.49560\end{array}$ & $\begin{array}{r}-10.2 \\
5.4 \\
8.8\end{array}$ \\
\hline $\begin{array}{l}\text { MP2/6-311+G(d,p) } \\
\text { //B3LYP/6-31+G(d) }\end{array}$ & $\begin{array}{l}\mathrm{E} \\
G^{\circ} \\
G^{\circ} \text { DMSO) }\end{array}$ & $\begin{array}{l}-271.04404 \\
-270.93400 \\
-270.94148\end{array}$ & $\begin{array}{l}-211.98188 \\
-211.87994 \\
-211.88643\end{array}$ & $\begin{array}{l}-483.04494 \\
-482.80775 \\
-482.81705\end{array}$ & $\begin{array}{r}-11.9 \\
3.9 \\
6.8\end{array}$ \\
\hline $\begin{array}{l}\text { CCSD(T)/def2-TZVP } \\
/ / B 3 L Y P / 6-311+G(d, p)\end{array}$ & $\begin{array}{l}\mathrm{E} \\
G^{\circ} \\
G^{\circ} \text { DMSO) }\end{array}$ & $\begin{array}{l}-270.92367 \\
-270.81363 \\
-270.82203\end{array}$ & $\begin{array}{l}-211.89247 \\
-211.79053 \\
-211.79744\end{array}$ & $\begin{array}{l}-482.82917 \\
-482.59198 \\
-482.60159\end{array}$ & $\begin{array}{r}-8.2 \\
7.6 \\
11.2\end{array}$ \\
\hline $\begin{array}{l}\operatorname{CCSD}(T) / 6-311+G(d, p) \\
/ / B 3 L Y P / 6-311+G(d, p)\end{array}$ & $\begin{array}{l}\mathbf{E} \\
\mathbf{G}^{\circ} \\
\boldsymbol{G}^{\circ}(\mathrm{DMSO})\end{array}$ & $\begin{array}{l}-271.10149 \\
-270.99273 \\
-271.00037\end{array}$ & $\begin{array}{l}-212.03512 \\
-211.93412 \\
-211.94030\end{array}$ & $\begin{array}{l}-483.15164 \\
-482.91635 \\
-482.92525\end{array}$ & $\begin{array}{r}-9.4 \\
6.6 \\
9.7\end{array}$ \\
\hline
\end{tabular}

- B2LYP-D3 improves B3LYP/6-311+G(d,p) but not enough.

- The reaction energies calculated with the $6-311+G(d, p)$ basis set are around $1 \mathrm{kcal} / \mathrm{mol}$ lower than those predicted by def2TZVP (see the B2LYP-D3 and CCSD(T) rows).

Optimized structures and Cartesian coordinates of the stationary points, at the B3LYP/6-311+G(d,p) level<smiles>CC(C)(C)C=O</smiles>

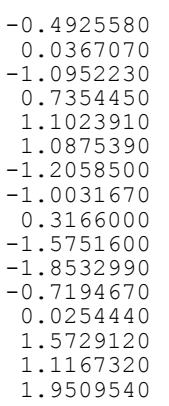

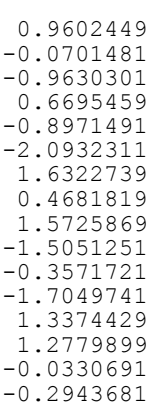<smiles>CC(C)(C)C(O)N1CCCC1</smiles>

HA-6a

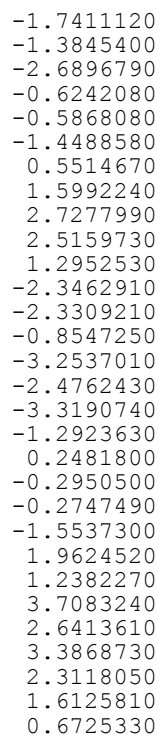

$$
\begin{array}{r}
0.1028220 \\
-0.8619890 \\
-1.3963100 \\
-2.0913240 \\
-0.1361480 \\
0.7135140 \\
0.6875220 \\
0.1200430 \\
1.1666360 \\
1.9127040 \\
1.2259620 \\
-0.4133900 \\
0.9499170 \\
0.5033470 \\
-1.9644970 \\
-2.0651130 \\
-0.5894560 \\
-2.6804280 \\
-1.8376750 \\
-2.7420360 \\
-0.9102420 \\
1.5225110 \\
-0.8470670 \\
-0.0408100 \\
0.6910150 \\
1.8566310 \\
1.8524420 \\
2.9709580 \\
0.4029780 \\
1.8974840
\end{array}
$$

1.5102220 0.3657310 $-0.2668130$ 0.9095880 $-1.5281800$ $-0.3667670$ 0.4933760 0.4468290 0.8967400 $-1.5234240$ 2. 2616170 1.1476980 2.0059990 0.4784560 1.1067200 0.6407380 1. 5445540 1. 5094120 0.0929240 $-1.4942630$ $-1.0118600$ 0.1038930 1.5078250 0.5149810 .2887090 1.5524480 $-0.7216820$ $-2.1903370$ 
Table 5. Calcd $\Delta G^{\circ}$ Values for the Reactions of Methyl Glyoxylate (7) with Pyrrolidine

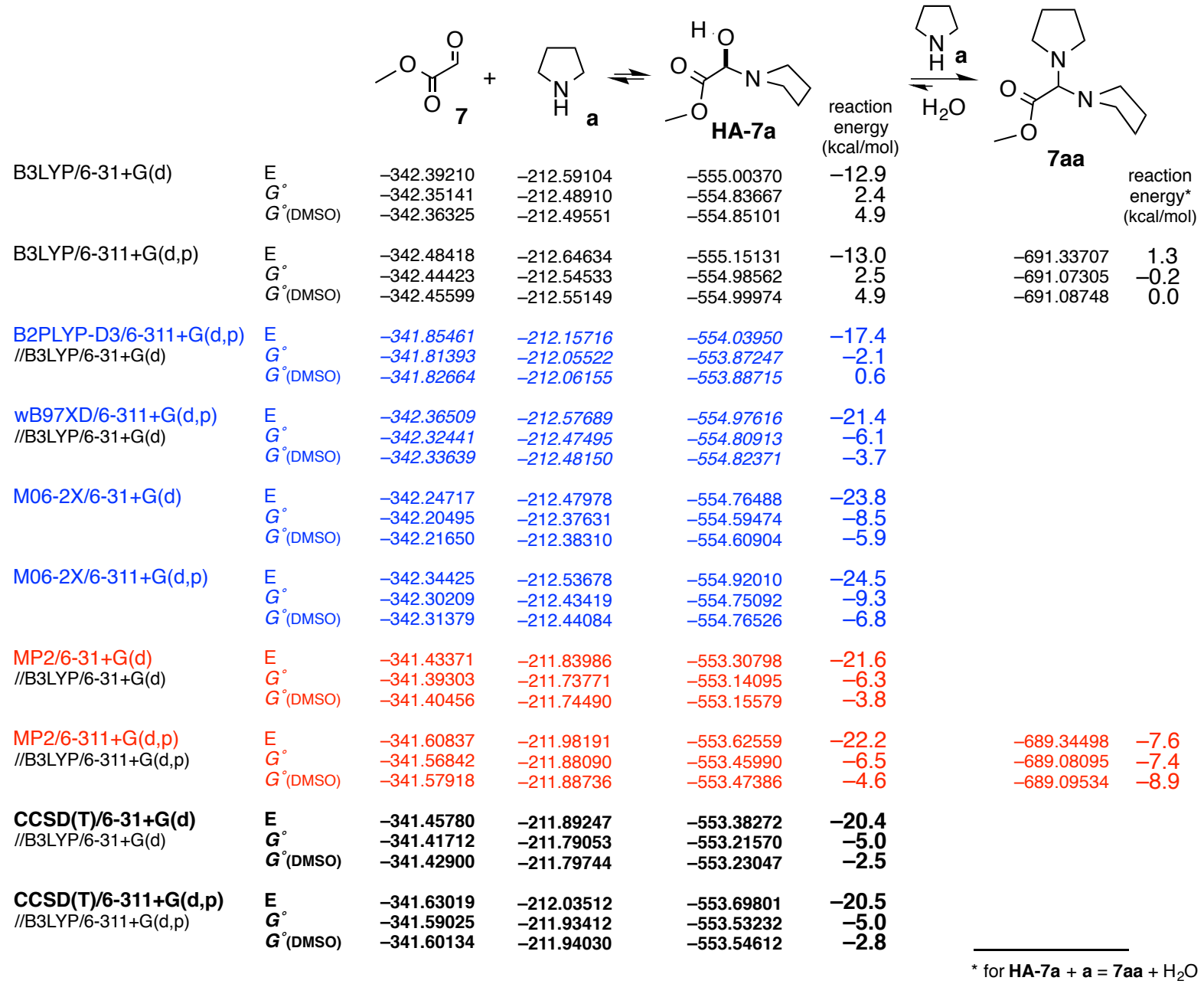

Optimized structures and Cartesian coordinates of the stationary points, at the B3LYP/6-31+G(d) level<smiles>COC(=O)C=O</smiles>

$\begin{array}{lrrr}\mathrm{C} & -1.6773200 & -0.3167139 & -0.0000001 \\ \mathrm{O} & -0.4782130 & 0.4880201 & -0.0000001 \\ \mathrm{C} & 0.6724890 & -0.1751729 & -0.0000001 \\ \mathrm{O} & 0.8043350 & -1.3754789 & -0.0000001 \\ \mathrm{C} & 1.8987810 & 0.7511431 & -0.0000001 \\ \mathrm{O} & 1.8491530 & 1.9481331 & -0.0000001 \\ \mathrm{H} & 2.8446300 & 0.1774561 & -0.0000001 \\ \mathrm{H} & -2.4995790 & 0.3938851 & -0.0000001 \\ \mathrm{H} & -1.7071380 & -0.9456359 & 0.8903959 \\ \mathrm{H} & -1.7071380 & -0.9456359 & -0.8903951\end{array}$

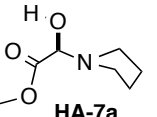

HA-7a

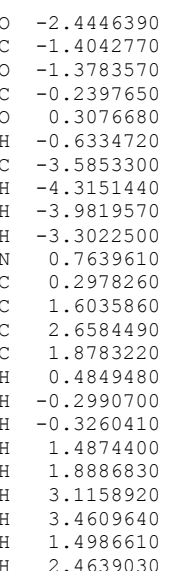

0.1655970 0.1243790 $-0.5179260$ 1.0166340 1.6660620 1.8159730 $-0.6435080$ $-0.4779280$ $-0.3303160$ $-1.6956450$ 0.2095910 $-0.6217030$ $-1.1058560$ $-0.0306260$ 0.9949420 0.9813020 $-0.0323950$ $-1.4436390$ $-1.2130860$ $-2.0814640$ $-2.0814640$ 0.4375710 $-0.4752290$ 1.4457220<smiles>COC(=O)C(NC1CCC1)N1CCCC1</smiles>

$7 a$

0.4702630 1.3156170 2.3358630 0.8520950 1.9730400 0.2056490 0.8264860 0.0376680 1.7926370 0.8767970 0.1810260 $-0.9379070$ $-1.5931960$ $-1.2091840$ 2.6336310 $-1.6560360$ $-0.5807300$ $-2.6733940$ $-1.1954660$ $-2.0828460$ $-0.6179470$ 0124480 0.4291550 
Addendum to Table 5. Formation of Hemiaminal HA-7b from Methyl Glyoxylate (Methyl 2-Oxoacetate) and Prolinol b (the Jorgensen-Hayashi catalyst)

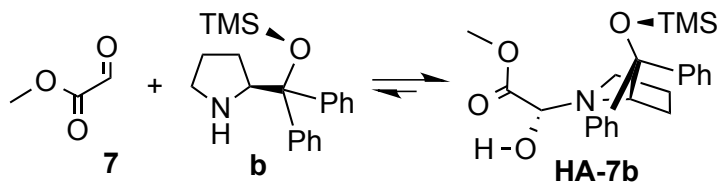

$\Delta \mathrm{E} \quad \Delta G^{\circ} \Delta G^{\circ}(\mathrm{DMSO})$

B3LYP/6-31G(d)

$B 3 L Y P / 6-31+G(d)$

B3LYP/6-311+G(d,p)

M06-2X/6-311+G(d,p)//B3LYP/6-311+G(d.p)

$$
-8 .
$$

$5.5 \quad 8.6$

$\begin{array}{lll}-8.6 & 7.7 & 12.1\end{array}$

$M P 2 / 6-311+G(d, p) / / B 3 L Y P / 6-311+G(d . p)$

$-22.2-6.0-1.9$

$-23.4-7.1-3.8$

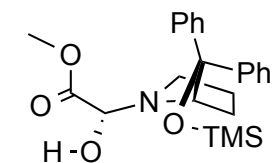

another conformer of $\mathbf{H A - 7 b}$

$$
\text { B3LYP/6-31G(d) }
$$$$
\Delta \mathrm{E}
$$

$$
\text { for EtOcocho (7') } K_{\text {eq }}>7 \cdot 10^{3} \mathrm{~L} \cdot \mathrm{mol}^{-1}\left(\mathrm{C}_{6} \mathrm{D}_{6}\right) \quad \Delta \mathrm{G}^{\circ}{ }_{\text {exptl }}<-5.2 \mathrm{kcal} / \mathrm{mol}
$$

- In agreement with other cases (SI-9) the conformer depicted at right is $2.2-2.5 \mathrm{kcal} / \mathrm{mol}$ more stable than the first. The corresponding

\begin{tabular}{|c|c|c|c|}
\hline C & -2.1998927 & 0.7055834 & -2.1756254 \\
\hline $\mathrm{H}$ & -2.3020297 & 0.0049214 & -3.0155834 \\
\hline $\mathrm{C}$ & 0.0574073 & 1.5981314 & -2.0423214 \\
\hline C & -0.0137647 & 0.1808964 & -1.4325344 \\
\hline $\mathrm{H}$ & 0.3143293 & 2.3102814 & -1.2613664 \\
\hline $\mathrm{H}$ & 0.8364613 & 1.6508624 & -2.8017204 \\
\hline $\mathrm{N}$ & -1.4562757 & 0.0704174 & -1.0651184 \\
\hline C & 1.0369963 & 0.0266194 & -0.2807794 \\
\hline $\mathrm{C}$ & 2.4106223 & 0.3739784 & -0.9130414 \\
\hline $\mathrm{C}$ & 1.1980243 & -1.3731666 & 0.3478726 \\
\hline $\mathrm{C}$ & 2.9795193 & -0.4723106 & -1.8720644 \\
\hline $\mathrm{C}$ & 3.1026213 & 1.5293704 & -0.5464174 \\
\hline C & 4.2022513 & -0.1609086 & -2.4614744 \\
\hline $\mathrm{C}$ & 4.8856593 & 0.9976264 & -2.0930894 \\
\hline C & 4.3319893 & 1.8378254 & -1.1305964 \\
\hline $\mathrm{C}$ & 0.7711623 & -2.5524006 & -0.2704764 \\
\hline $\mathrm{C}$ & 1.0107273 & -3.7950456 & 0.3256756 \\
\hline $\mathrm{C}$ & 1.6908083 & -3.8804966 & 1.5353526 \\
\hline $\mathrm{C}$ & 2.1525023 & -2.7085886 & 2.1426086 \\
\hline $\mathrm{C}$ & 1.9167523 & -1.4736056 & 1.5518286 \\
\hline 0 & 0.8220733 & 1.0109224 & 0.7269986 \\
\hline Si & -0.2531327 & 1.3576984 & 1.9728796 \\
\hline $\mathrm{C}$ & 0.8270043 & 2.1841714 & 3.2794986 \\
\hline $\mathrm{C}$ & -1.0914537 & -0.1253276 & 2.7752566 \\
\hline $\mathrm{C}$ & -1.5434397 & 2.6055114 & 1.4062516 \\
\hline $\mathrm{H}$ & 2.4753573 & -1.3889756 & -2.1555394 \\
\hline $\mathrm{H}$ & 2.6746243 & 2.1818004 & 0.2023326 \\
\hline $\mathrm{H}$ & 4.6255253 & -0.8308496 & -3.2018634 \\
\hline $\mathrm{H}$ & 5.8410943 & 1.2368344 & -2.5464044 \\
\hline $\mathrm{H}$ & 4.8556293 & 2.7390654 & -0.8301764 \\
\hline $\mathrm{H}$ & 0.2699063 & -2.5295926 & -1.2343274 \\
\hline $\mathrm{H}$ & 0.6632383 & -4.6942506 & -0.1709424 \\
\hline $\mathrm{H}$ & 1.8739633 & -4.8453946 & 1.9945976 \\
\hline $\mathrm{H}$ & 2.7049913 & -2.7585456 & 3.0747066 \\
\hline $\mathrm{H}$ & 2.2929873 & -0.5747096 & 2.0223926 \\
\hline $\mathrm{H}$ & 1.3229493 & 3.0763714 & 2.8861656 \\
\hline $\mathrm{H}$ & 1.6019833 & 1.5070974 & 3.6497656 \\
\hline $\mathrm{H}$ & 0.2134723 & 2.4911714 & 4.1324156 \\
\hline $\mathrm{H}$ & -0.3667827 & -0.8054606 & 3.2295066 \\
\hline $\mathrm{H}$ & -1.7125417 & -0.6971756 & 2.0852186 \\
\hline $\mathrm{H}$ & -1.7478357 & 0.2471964 & 3.5693306 \\
\hline $\mathrm{H}$ & -1.0759207 & 3.4660934 & 0.9179376 \\
\hline $\mathrm{H}$ & -2.1002407 & 2.9793294 & 2.2723116 \\
\hline $\mathrm{H}$ & -2.2528567 & 2.1554484 & 0.7090886 \\
\hline $\mathrm{H}$ & 0.2296503 & -0.5500436 & -2.2133424 \\
\hline $\mathrm{C}$ & -1.3516287 & 1.9094004 & -2.5944584 \\
\hline $\mathrm{H}$ & -1.3598077 & 2.0385754 & -3.6781554 \\
\hline $\mathrm{H}$ & -1.7414197 & 2.8299994 & -2.1556164 \\
\hline $\mathrm{H}$ & -3.2017887 & 0.9837384 & -1.8356694 \\
\hline C & -1.9600617 & -1.2470366 & -0.7928574 \\
\hline $\mathrm{H}$ & -1.4166547 & -1.6524786 & 0.0672536 \\
\hline $\mathrm{C}$ & -3.4349627 & -1.2213756 & -0.3747534 \\
\hline 0 & -3.7173247 & -0.2407366 & 0.4779146 \\
\hline 0 & -4.2161677 & -2.0777586 & -0.7287574 \\
\hline 0 & -1.8143857 & -2.1527106 & -1.8843004 \\
\hline $\mathrm{H}$ & -2.6457787 & -2.6419196 & -1.9646344 \\
\hline $\mathrm{C}$ & -5.0637657 & -0.2209546 & 0.9927006 \\
\hline H & -5.7847447 & -0.2969216 & 0.178876 \\
\hline $\mathrm{H}$ & -5.2095847 & -1.0494726 & 1.6875846 \\
\hline $\mathrm{H}$ & -5.1580387 & 0.7312734 & 1.509686 \\
\hline
\end{tabular}
$\Delta G^{\circ}$ values, not calculated, are expected to be around $2.5 \mathrm{kcal} / \mathrm{mol}$ lower.

Cartesian coordinates of $\boldsymbol{H A - 7 b}$, at the B3LYP/6-311+G(d,p) level 
Table 6. Calcd vs Exptl $\Delta G^{\circ}$ Values for the Formation of Aminal 5aa from 5a and Pyrrolidine

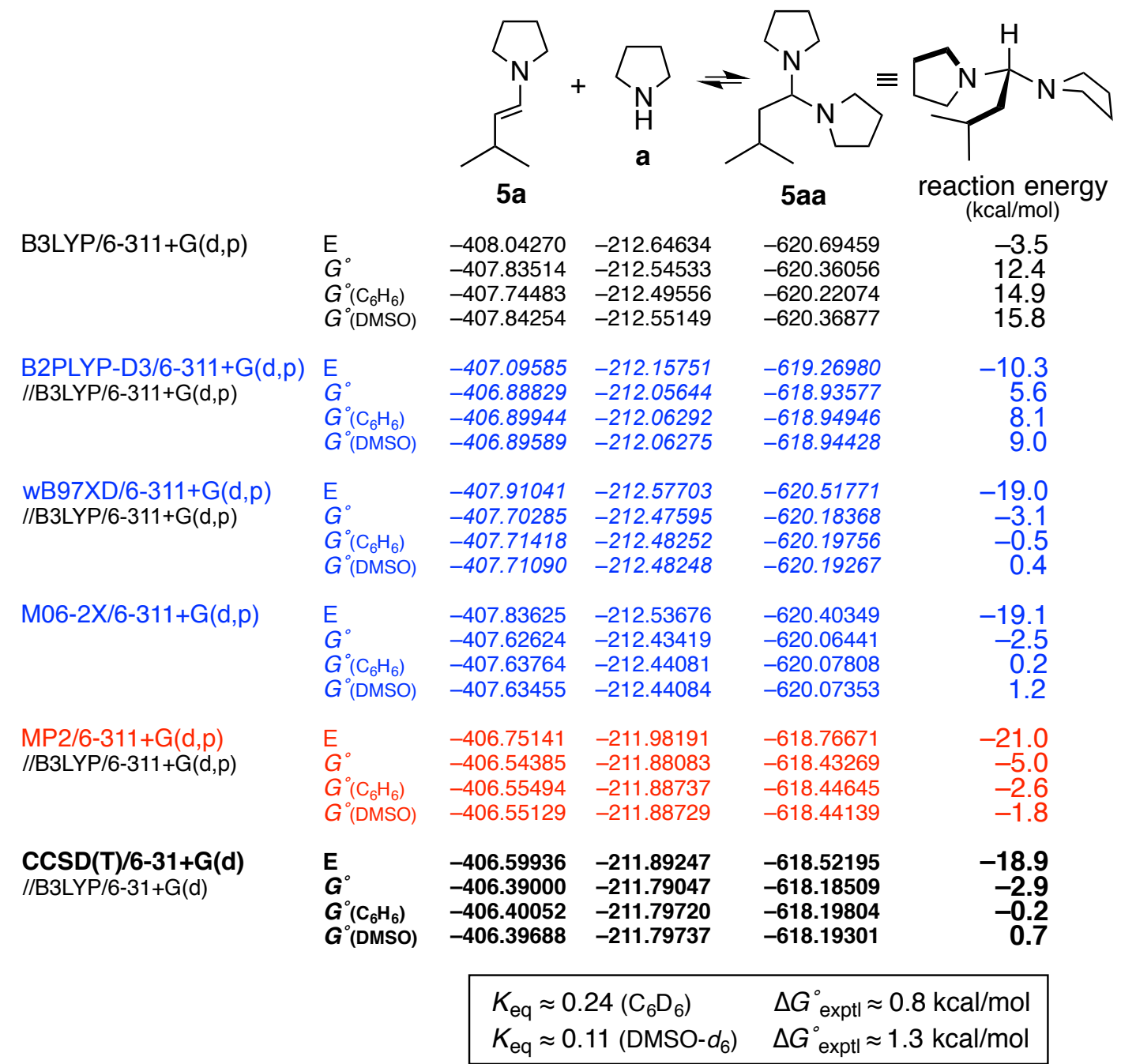

- Further results with smaller basis sets (not included in the Table to save space): B3LYP/6-31G(d), $\Delta \mathrm{E}=-11.1 \mathrm{kcal} / \mathrm{mol}, \Delta \mathrm{G}^{\circ}=4.3 \mathrm{kcal} / \mathrm{mol}$ $\mathrm{B} 3 \mathrm{~L} Y \mathrm{P} / 6-31+\mathrm{G}(\mathrm{d}), \Delta \mathrm{E}=-7.5 \mathrm{kcal} / \mathrm{mol}, \Delta G^{\circ}=7.8 \mathrm{kcal} / \mathrm{mol}$

- With these small basis sets the $\Delta \mathrm{E}$ values are more negative (and thus the $\Delta G^{\circ}$ values are not so high as those predicted by B3LYP/6-311+ $\mathrm{G}(\mathrm{d}, \mathrm{p})$ ), due to the poorer description of the electronic delocalization of $5 \mathrm{a}$. However, this error does not compensate the lack of inclusion of dispersion. Only in cases as this one B3LYP with small basis sets may overcome B3LYP with large basis sets.

- At the M06-2X/6-31+G(d) level, $\Delta \mathrm{E}=-21.1 \mathrm{kcal} / \mathrm{mol}$ and $\Delta G^{\circ}=-5.6 \mathrm{kcal} / \mathrm{mol}$.

- Single-point calculations at the $\operatorname{CCSD}(T) / 6-31+G(d)$ level from other DFT geometries gave essentially the same result (max. diff. $1 \mathrm{kcal} / \mathrm{mol}$ ).

- We were unable to carry out a single-point $\operatorname{CCSD}(T) / 6-311+G(d, p)$ calculation of $5 a a$ (it did not converge).

Cartesian coordinates of the stationary point (minimum energy conformer), at the B3LYP/6-311+G(d,p) level

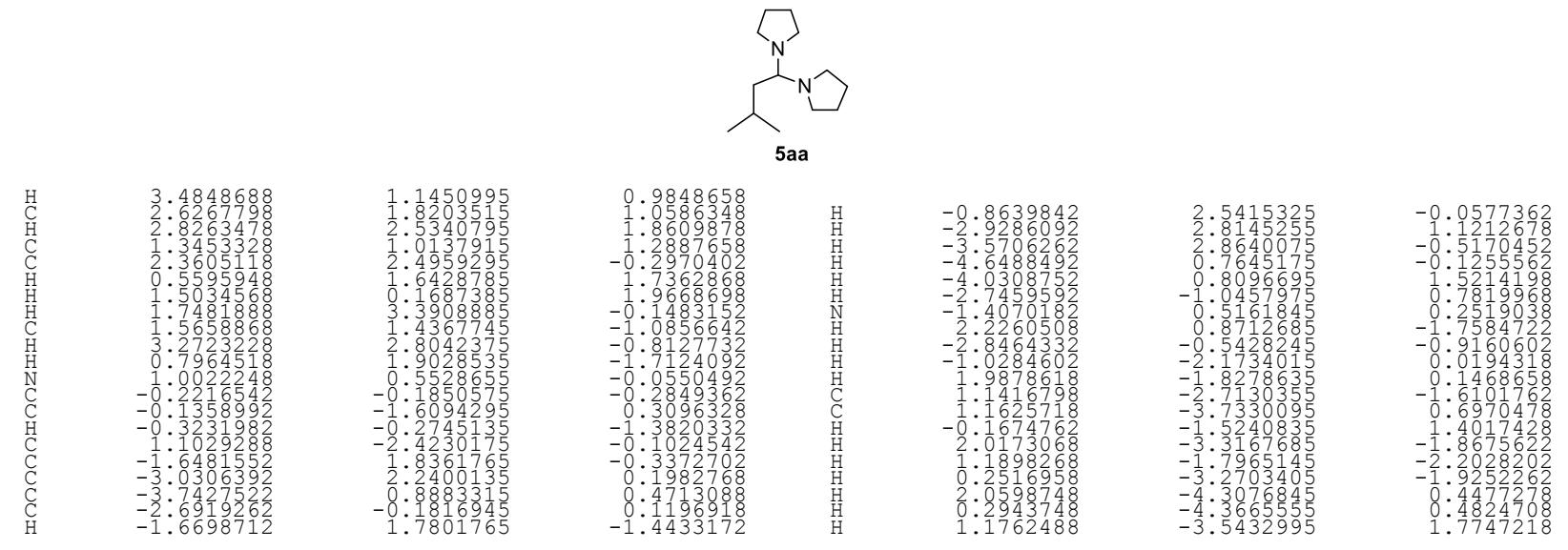


Table 7. Calcd vs. Exptl $\Delta G^{0}$ Values for the Exchange of the 1-Cyclohexenyl Group between Secondary Amines a and $\mathrm{c}$

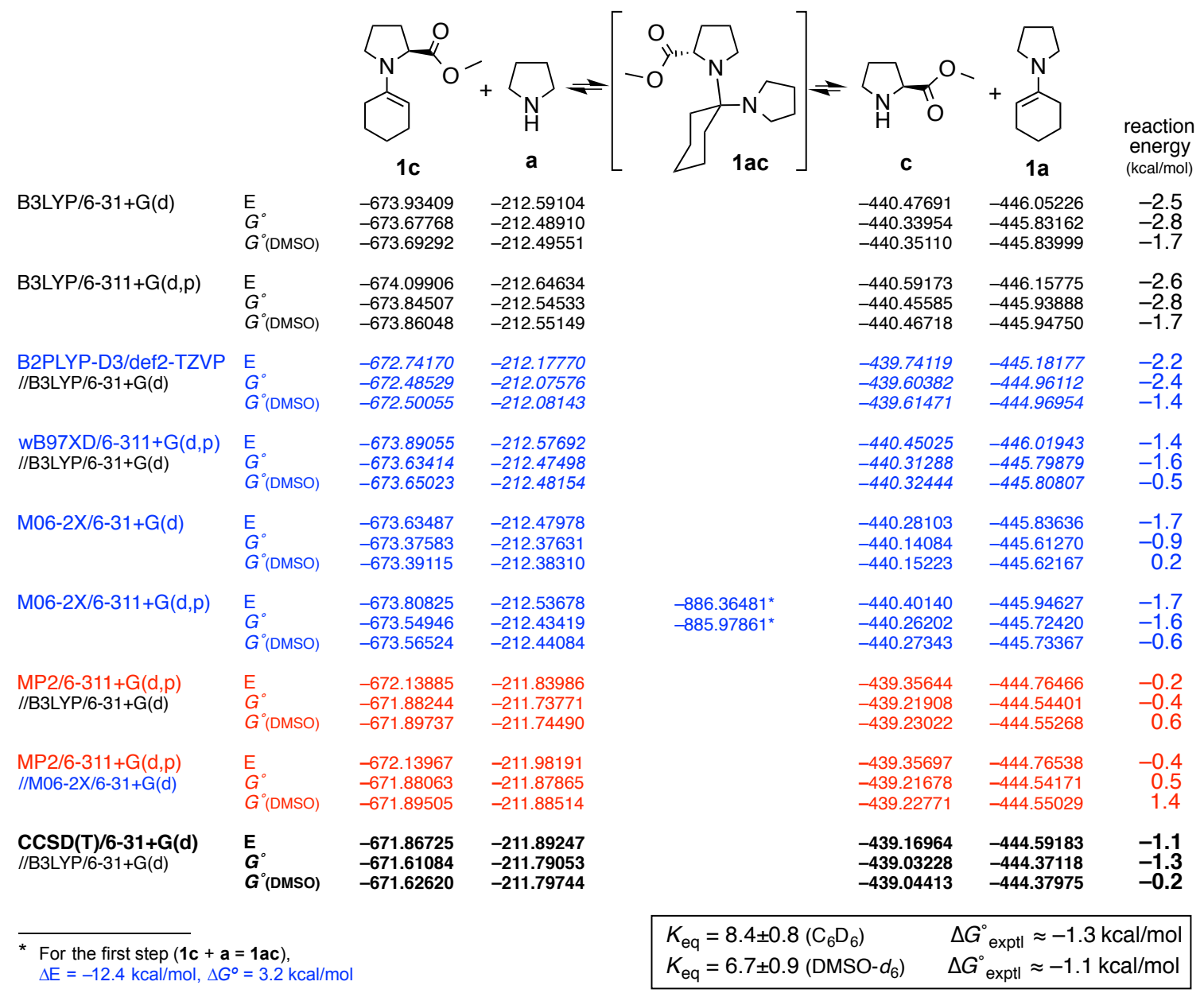

Optimized structures and Cartesian coordinates of the stationary points, at the B3LYP/6-31+G(d) level<smiles>COC(=O)C1CCCN1C1=CCCCC1</smiles>

1c

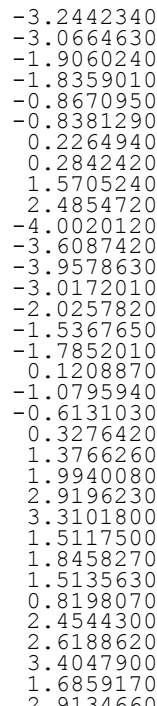<smiles>COC(=O)C1CCCN1</smiles>

c

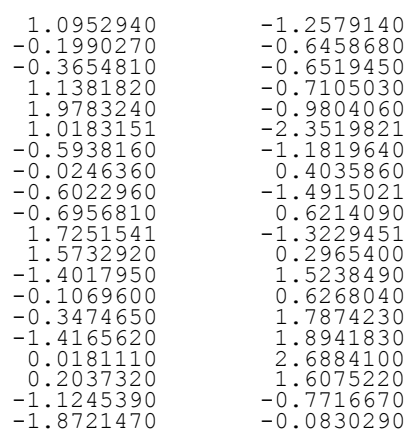


Optimized structures and Cartesian coordinates of the stationary points, at the B3LYP/6-311+G(d,p) level<smiles>COC(=O)C1CCCN1C1=CCCCC1</smiles>

$1 \mathrm{c}$

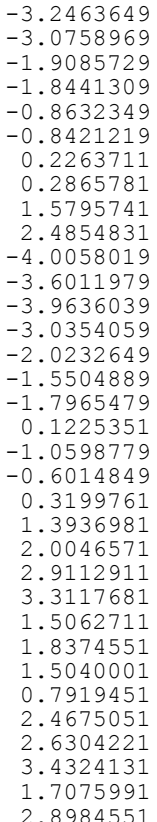

$-1.4886450$ $-0.2066610$

$-2.2220500$

0.5725250

$-1.3560530$

0.0772910

0.8647020

2. 2916780

2.7572980
1.5254750

1.5254750
-2.1343540

-2.1343540
-1.2338130

0.4273020

$-0.4636660$

$-3.1693880$

$-2.4695850$

1.5978600

$-1.8159360$

$-1.3456580$

2. 8003240

2.4673600
2.9917030
3.6420620

3.6420620

1.4620590

1.5113630

0.3385390

$-0.5211620$

$-0.0879990$

0.3484370

$-1.0124240$

$-1.4509240$

$-2.1855170$

$-1.9014300$

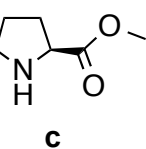

C

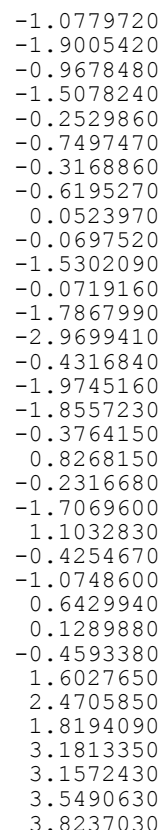

$-1.7452391$

$-2.3028371$

0.0890989

$-0.3235581$

$-2.3370851$

$-1.7112751$

$-3.1541941$

0.7532439

0.8666689

0.8666689

0.3746349
-0.3477751

-0.37463491
0.4674739

2. 0809349

2.0809349

2. 9116329

3.1126739
2.4242689

2.4242689
3.8329339

$-1.1594821$

$-1.2003121$

1.0904105

$-0.2014615$

$-0.3630685$

1.1368705

1.9705536

1.0107615

$-0.5972015$

$-0.0224245$

$-0.5928025$

$-0.6949235$

$-0.6949235$

1.7325135

1.7325135
-1.5581235
-1.3923015

$-1.3923015$

$-0.1112825$

$-0.3488565$

$-1.4152355$

0.0197055

0.1975935

$-1.8507235$

$-1.2673055$ $-0.6665725$ $-0.6353275$ $-0.6874465$ $-1.0077475$ $-2.3578575$ $-1.2263845$ 0.3690095 $-1.4769425$ 0.6295675 $-1.2760285$ 0.3222125 1.5322305 0.6200235 0.6200235 1.7730175 1.8831885
2.6766045 2.6766045 1.5842105 $-0.7481045$ $-0.0403475$

Table 8. Calcd vs. Exptl $\Delta G^{o}$ Values for the Exchange of an Alkenyl Group between Secondary Amines $a$ and $b$

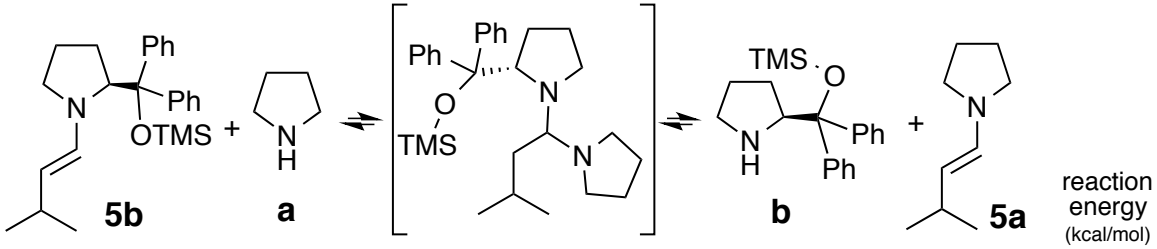

\begin{tabular}{|c|c|c|c|}
\hline 33LYP/6-31G(d) & $\begin{array}{l}\mathrm{E} \\
G^{\circ} \\
G^{\circ} \text { (DMSO) }\end{array}$ & $\begin{array}{l}-1393.25894 \\
-1392.77523 \\
-1392.78789\end{array}$ & $\begin{array}{l}-212.58173 \\
-212.47958 \\
-212.48473\end{array}$ \\
\hline B3LYP/6-31+G(d) & $\begin{array}{l}\mathrm{E} \\
G^{\circ} \\
G^{\circ}(\mathrm{DMSO})\end{array}$ & $\begin{array}{l}-1393.29416 \\
-1392.81189 \\
-1392.82592\end{array}$ & $\begin{array}{l}-212.59104 \\
-212.48910 \\
-212.49551\end{array}$ \\
\hline $33 L Y P / 6-311+G(d, p)$ & $\begin{array}{l}\mathrm{E} \\
G^{\circ} \\
G^{\circ}(\mathrm{DMSO})\end{array}$ & $\begin{array}{l}-1393.57827 \\
-1393.09977 \\
-1393.11400\end{array}$ & $\begin{array}{l}-212.64634 \\
-212.54533 \\
-212.55149\end{array}$ \\
\hline $\begin{array}{l}\text { M06-2X/6-311+G(d,p) } \\
\text { //B3LYP/6-311+G(d,p) }\end{array}$ & $\begin{array}{l}\mathrm{E} \\
G^{\circ} \\
G^{\circ}(\mathrm{DMSO})\end{array}$ & $\begin{array}{l}-1393.05759 \\
-1392.57911 \\
-1392.59604\end{array}$ & $\begin{array}{l}-212.53658 \\
-212.43557 \\
-212.44220\end{array}$ \\
\hline $\begin{array}{l}\text { AP2/6-311+G(d,p) } \\
\text { BBSLYP/6-311+G(d,p) }\end{array}$ & $\begin{array}{l}\mathrm{E} \\
G^{\circ} \\
G^{\circ} \text { (DMSO) }\end{array}$ & $\begin{array}{l}-1389.74188 \\
-1389.26340 \\
-1389.27769\end{array}$ & $\begin{array}{l}-211.98191 \\
-211.88090 \\
-211.88736\end{array}$ \\
\hline
\end{tabular}

$\begin{array}{llr}-212.58173 & -407.92970 & -4.8 \\ -212.47948 & -407.71952 & -5.4 \\ --212.48462 & -407.72605 & -5.3 \\ -212.59104 & -407.94317 & -4.3 \\ -212.48910 & -407.73376 & -5.6 \\ -212.49551 & -407.74104 & -5.0 \\ -212.64634 & -408.04270 & -4.6 \\ -212.54533 & -407.83514 & -5.3 \\ -212.55149 & -407.84254 & -4.8 \\ -212.53658 & -407.83580 & -2.2 \\ -212.43557 & -407.62824 & -2.9 \\ -212.44220 & -407.63663 & -2.6 \\ -211.98191 & -406.75141 & 1.7 \\ -211.88090 & -406.54385 & 1.0 \\ -211.88736 & -406.55129 & 1.6\end{array}$

$K_{\text {eq }} \approx 75\left(\mathrm{C}_{6} \mathrm{D}_{6}\right) \quad \Delta G_{\text {exptl }}^{\circ} \approx-2.6 \mathrm{kcal} / \mathrm{mol}$ 
NMR Spectra of Enamine 1a

${ }^{1} \mathrm{H} \operatorname{NMR}\left(\mathrm{C}_{6} \mathbf{D}_{6}\right)$

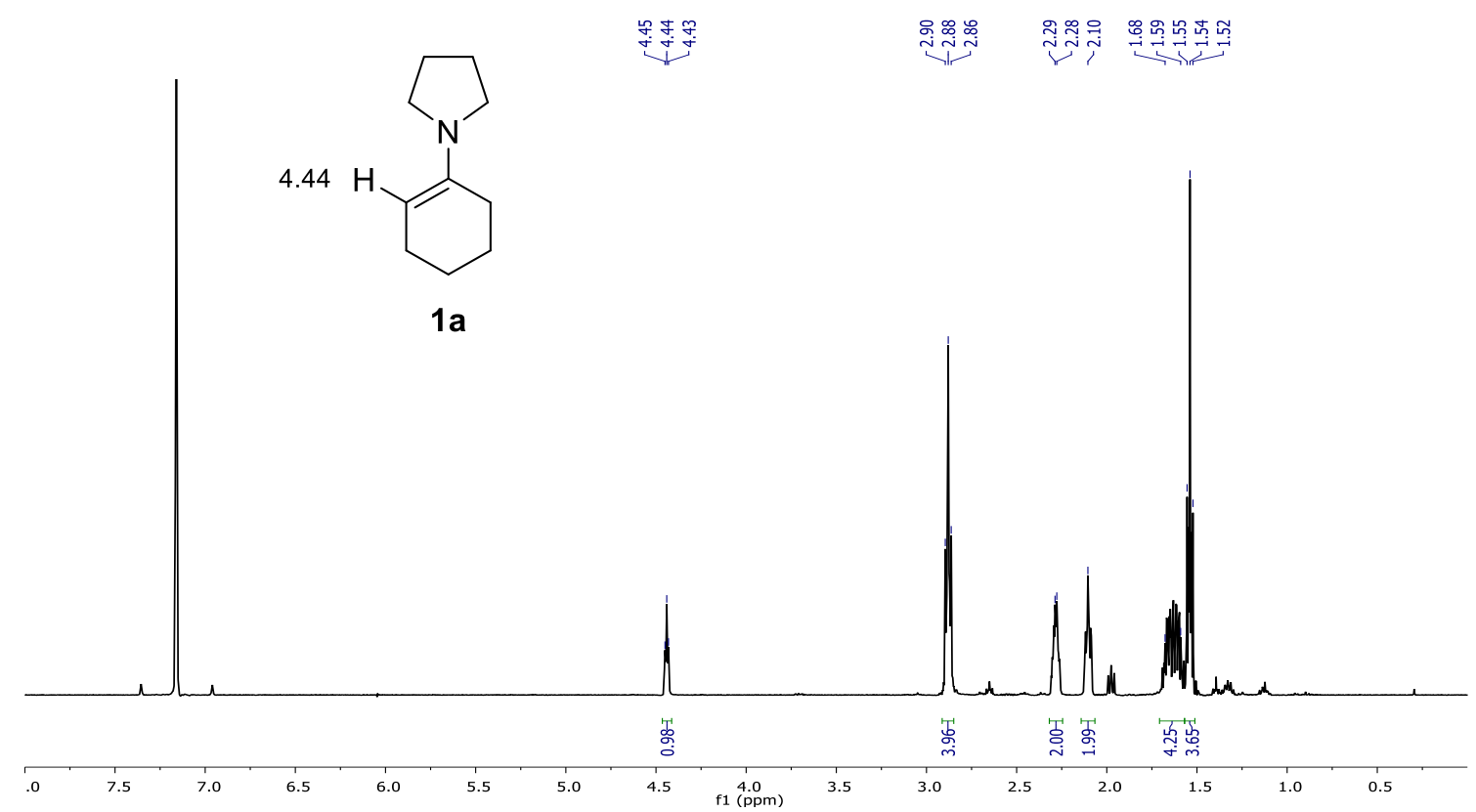

Enamine 1a: $\delta 4.44(\mathrm{t}, J=3.9 \mathrm{~Hz}, 1 \mathrm{H}), 2.88(\mathrm{~m}, 4 \mathrm{H}), 2.28(\mathrm{~m}, 2 \mathrm{H}), 2.10(\mathrm{~m}, 2 \mathrm{H}), 1.63$ $(\mathrm{m}, 4 \mathrm{H}), 1.54(\mathrm{~m}, 4 \mathrm{H})$.

See ref 24 of the main text for spectra in $\mathrm{C}_{6} \mathrm{D}_{6}$ and in $\mathrm{CDCl}_{3}$.

\section{${ }^{13} \mathrm{C}$ NMR $\left(\mathrm{C}_{6} \mathrm{D}_{6}\right)$}

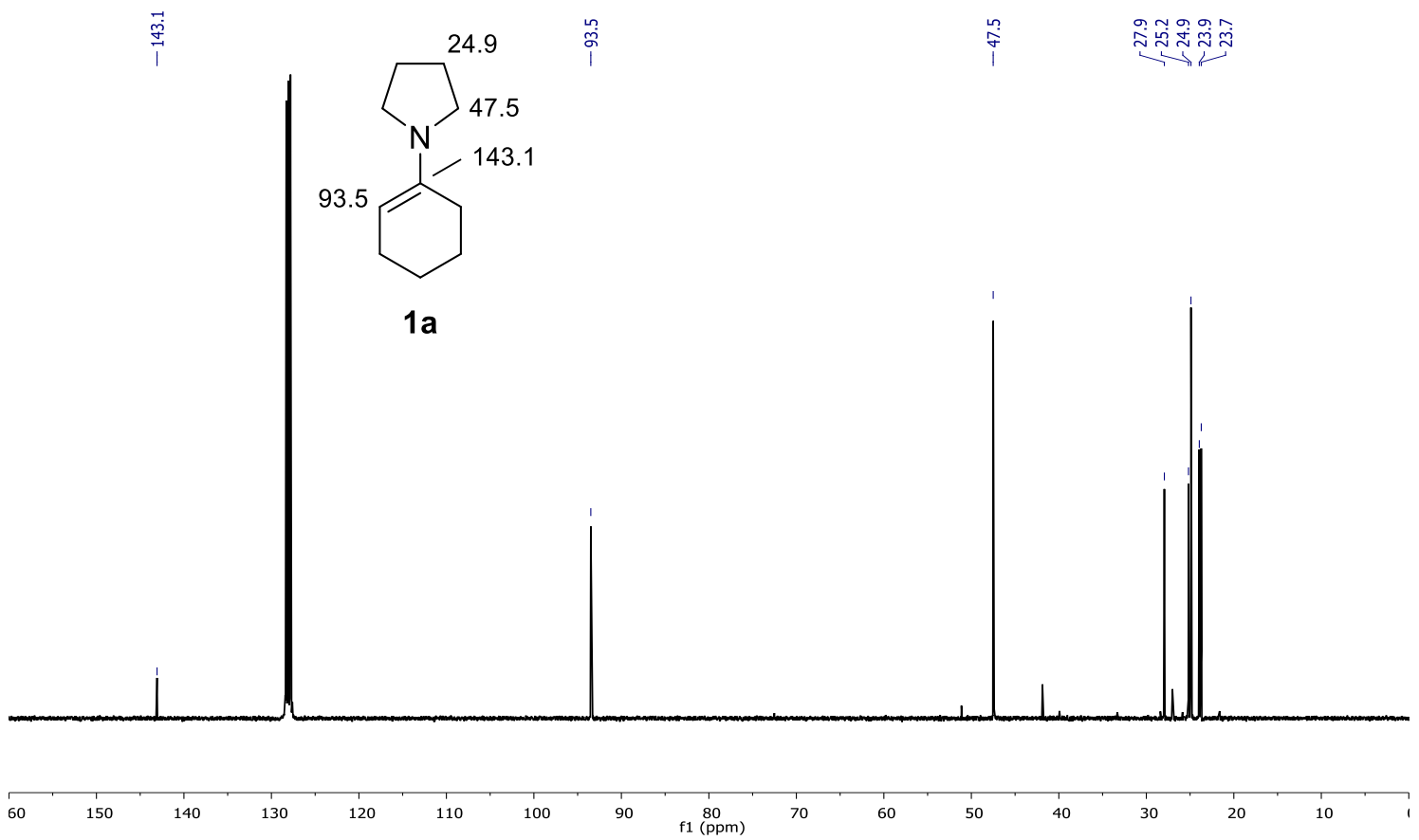

Enamine 1a: $\delta 143.1,93.5,47.5,27.9,25.2,24.9,23.9,23.7$. 


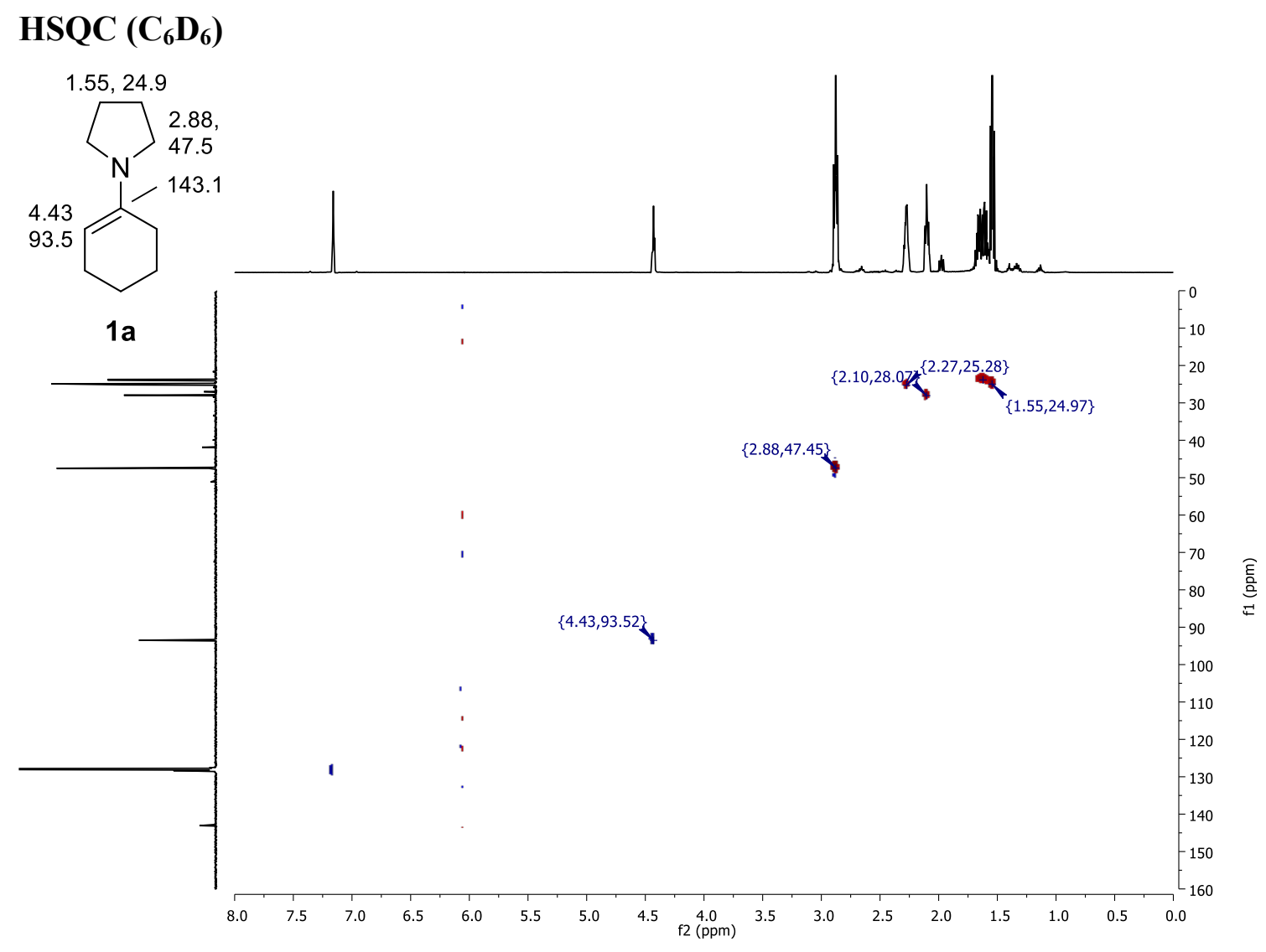

NMR Spectra of Enamine 2a

${ }^{1} H$ NMR $\left(C_{6} D_{6}\right)$

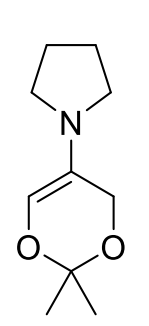

羿

$2 a$

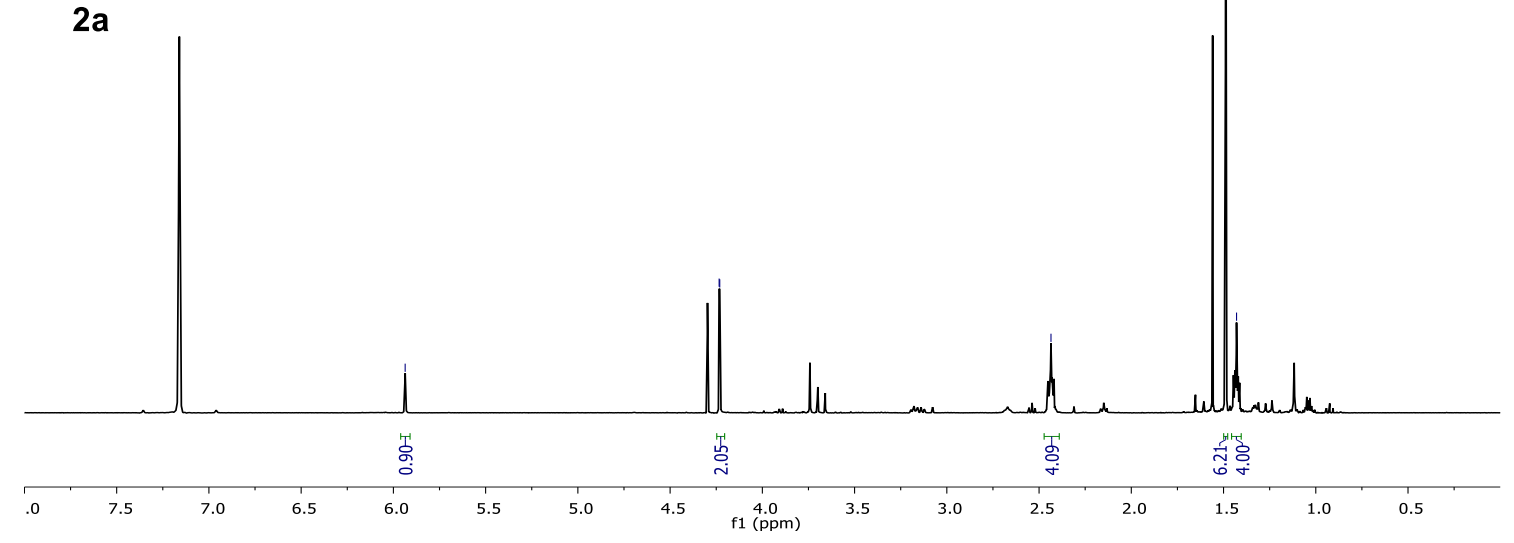

Enamine 2a: $\delta 5.94$ (br s, 1H), 4.23 (d, $J=1.3 \mathrm{~Hz}, 2 \mathrm{H}), 2.44$ (m, 4H), 1.49 (s, 6H), 1.43 (m, 4H). 
${ }^{13} \mathrm{C} \operatorname{NMR}\left(\mathbf{C}_{6} \mathrm{D}_{6}\right)$

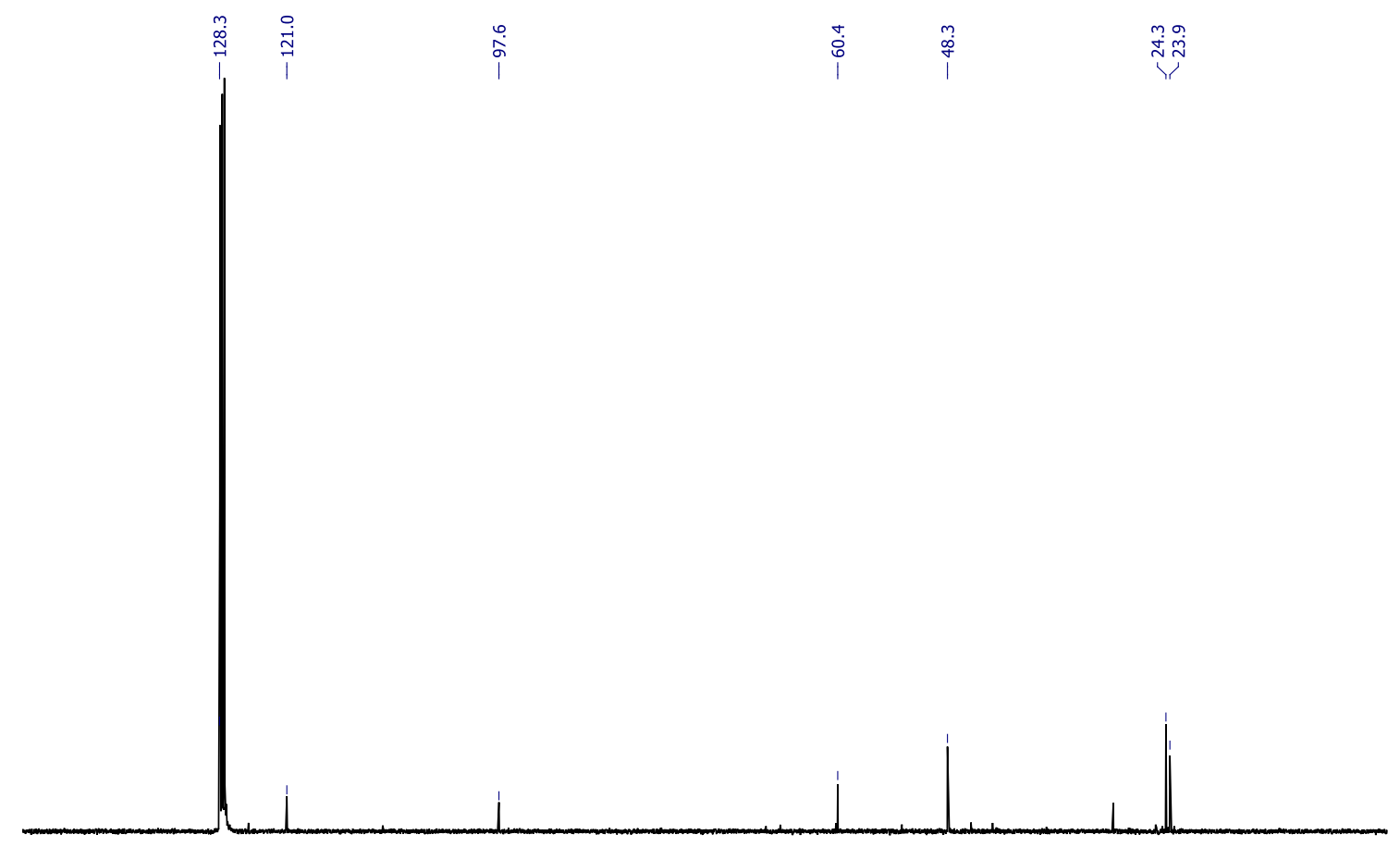

\begin{tabular}{llllllllllllllllllllllllllllllllllllll}
50 & 145 & 140 & 135 & 130 & 125 & 120 & 115 & 110 & 105 & 100 & 95 & 90 & 85 & 80 & $\begin{array}{c}75 \\
\mathrm{f} 1(\mathrm{pm})\end{array}$ & 70 & 65 & 60 & 55 & 50 & 45 & 40 & 35 & 30 & 25 & 20 & 15 & 10 & 5 & 1 \\
\hline
\end{tabular}

Enamine 2a: $\delta 128.3,121.0,97.6,60.4,48.3,24.3,23.9$.

\section{HSQC ( $\left.\mathbf{C}_{6} \mathbf{D}_{6}\right)$}

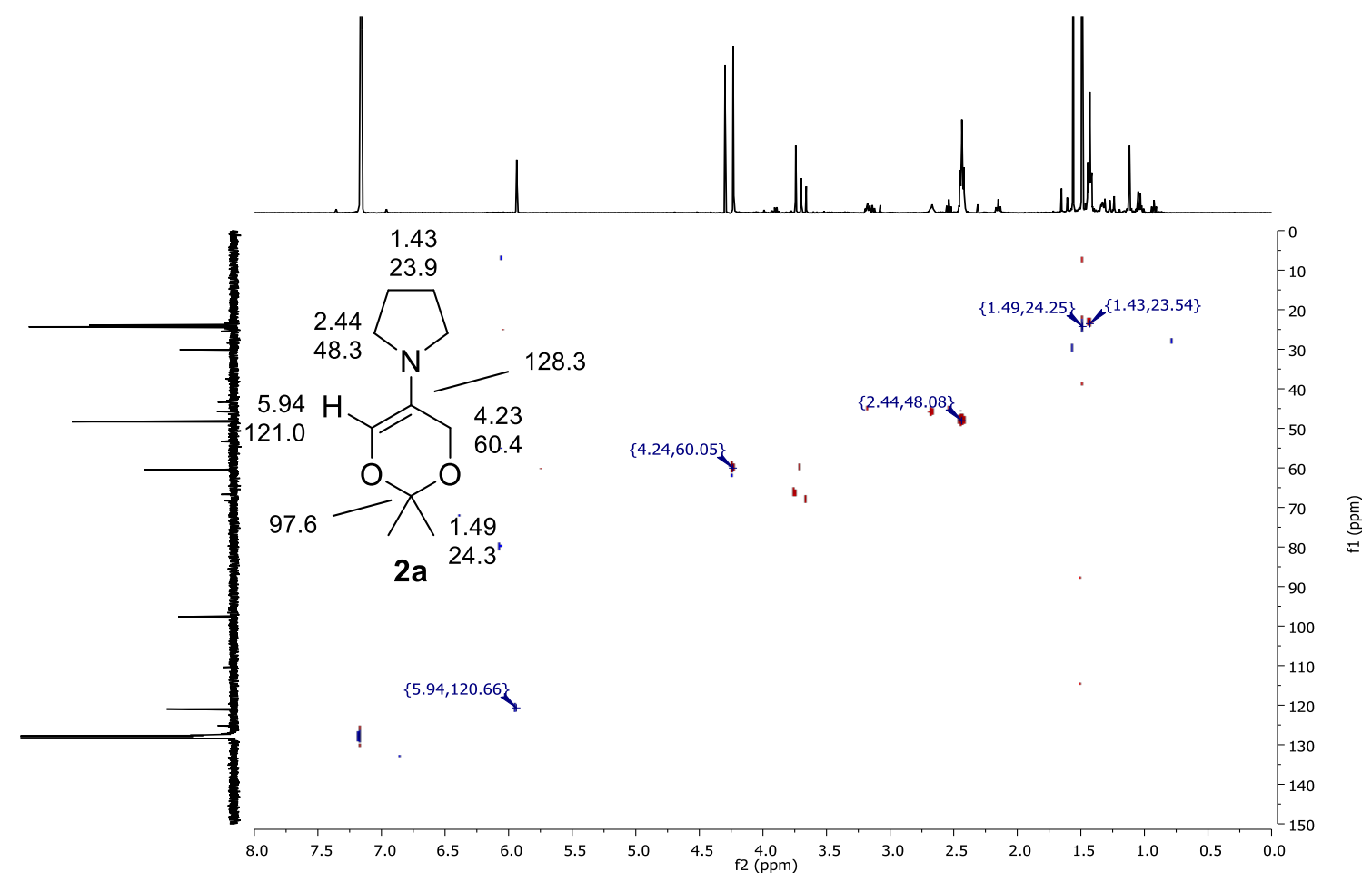


Reaction of 1 a with 2

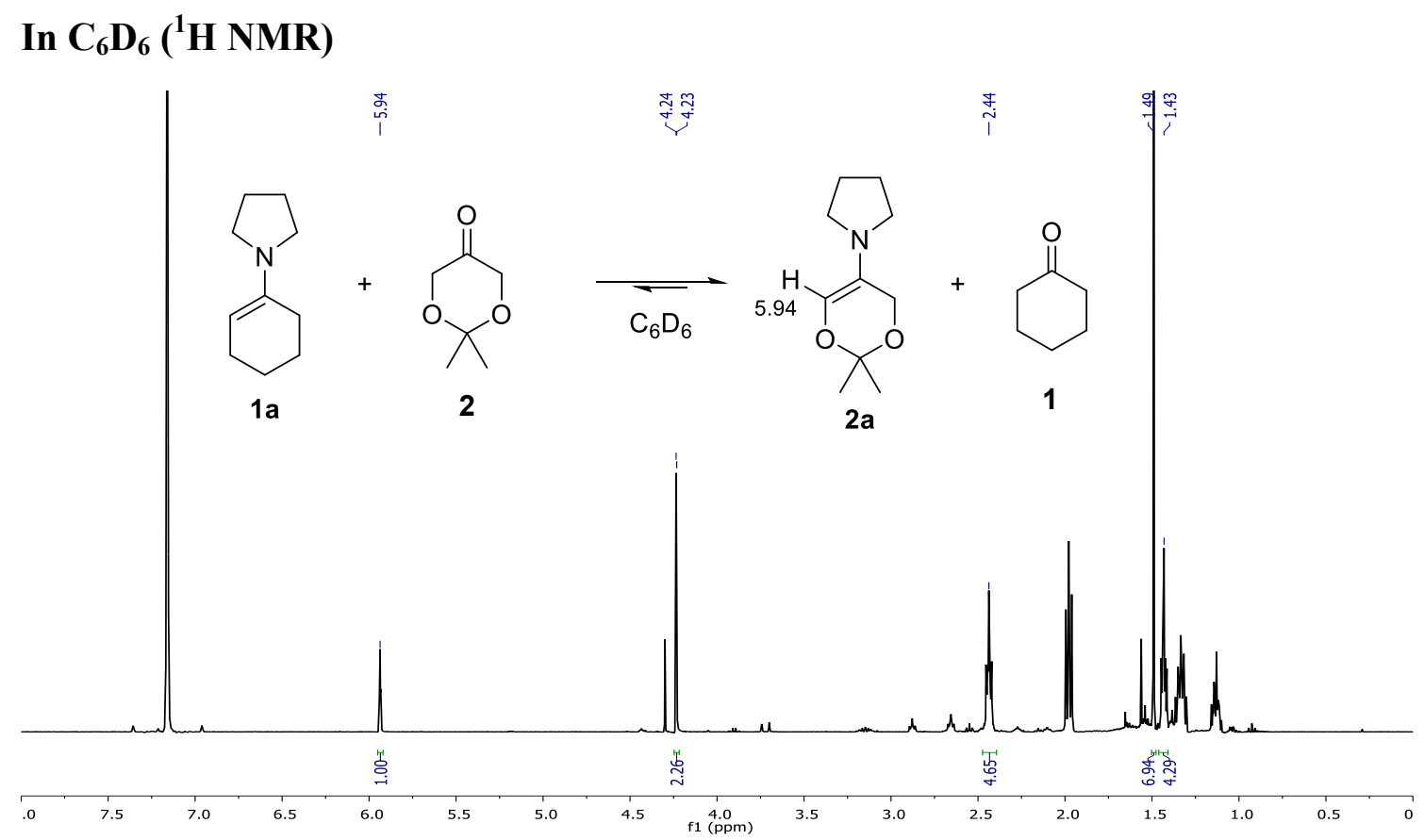

In DMSO- $d_{6}\left({ }^{1} \mathrm{H}\right.$ NMR)

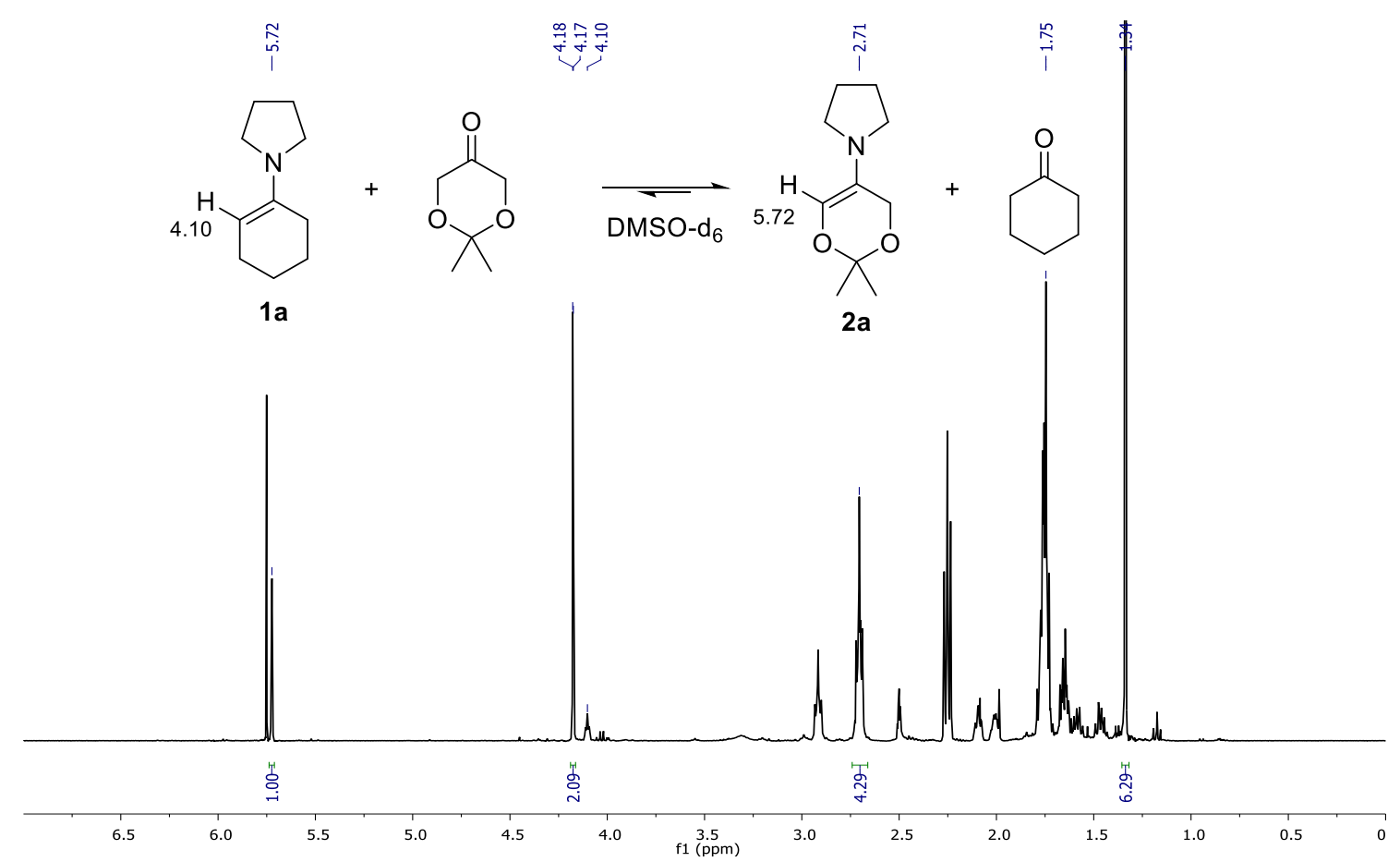


Reaction of 1 a with 3

In $\mathrm{C}_{6} \mathrm{D}_{6}\left({ }^{1} \mathrm{H}\right.$ NMR)

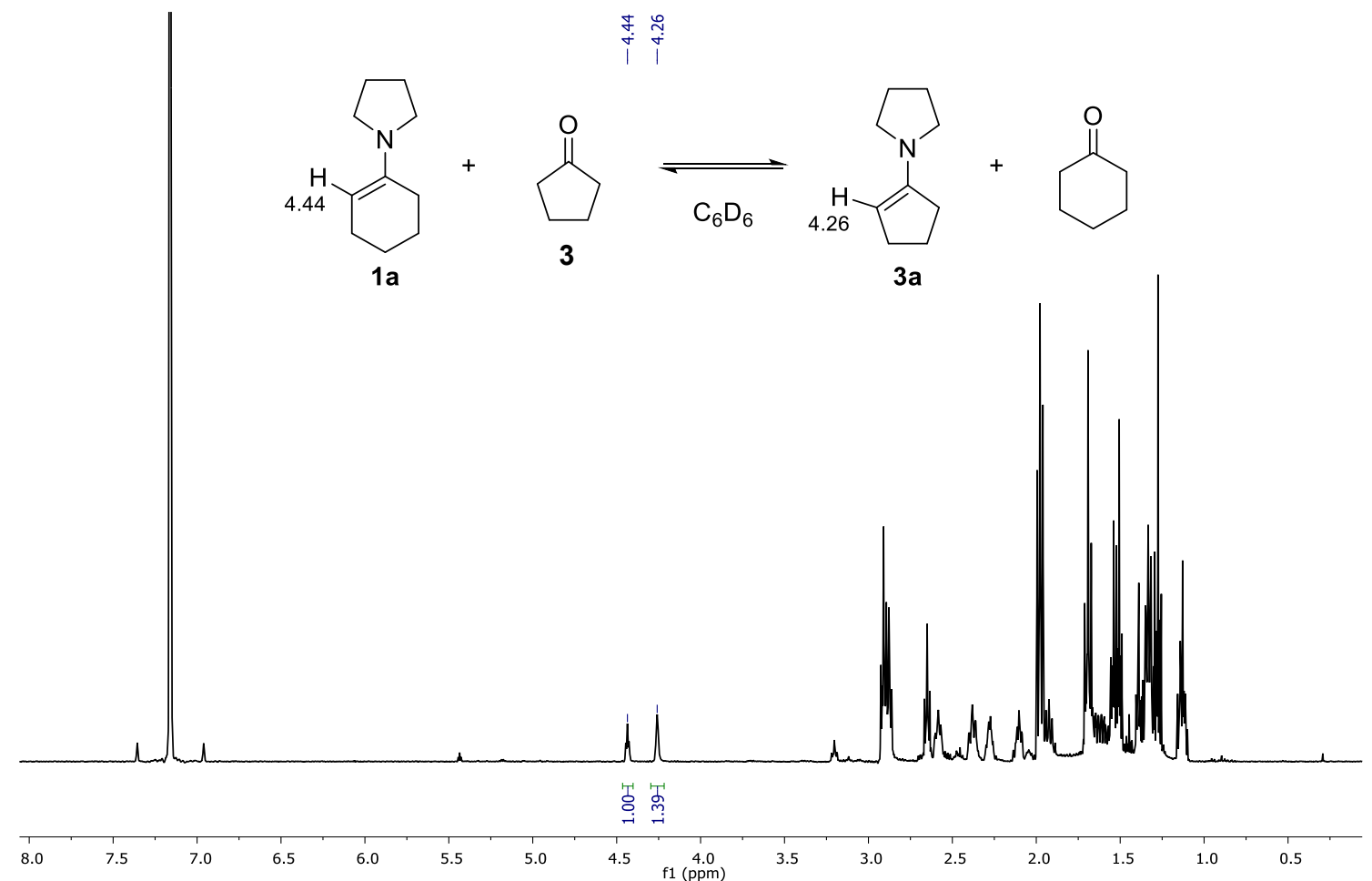

In DMSO- $d_{6}\left({ }^{1} \mathrm{H}\right.$ NMR)
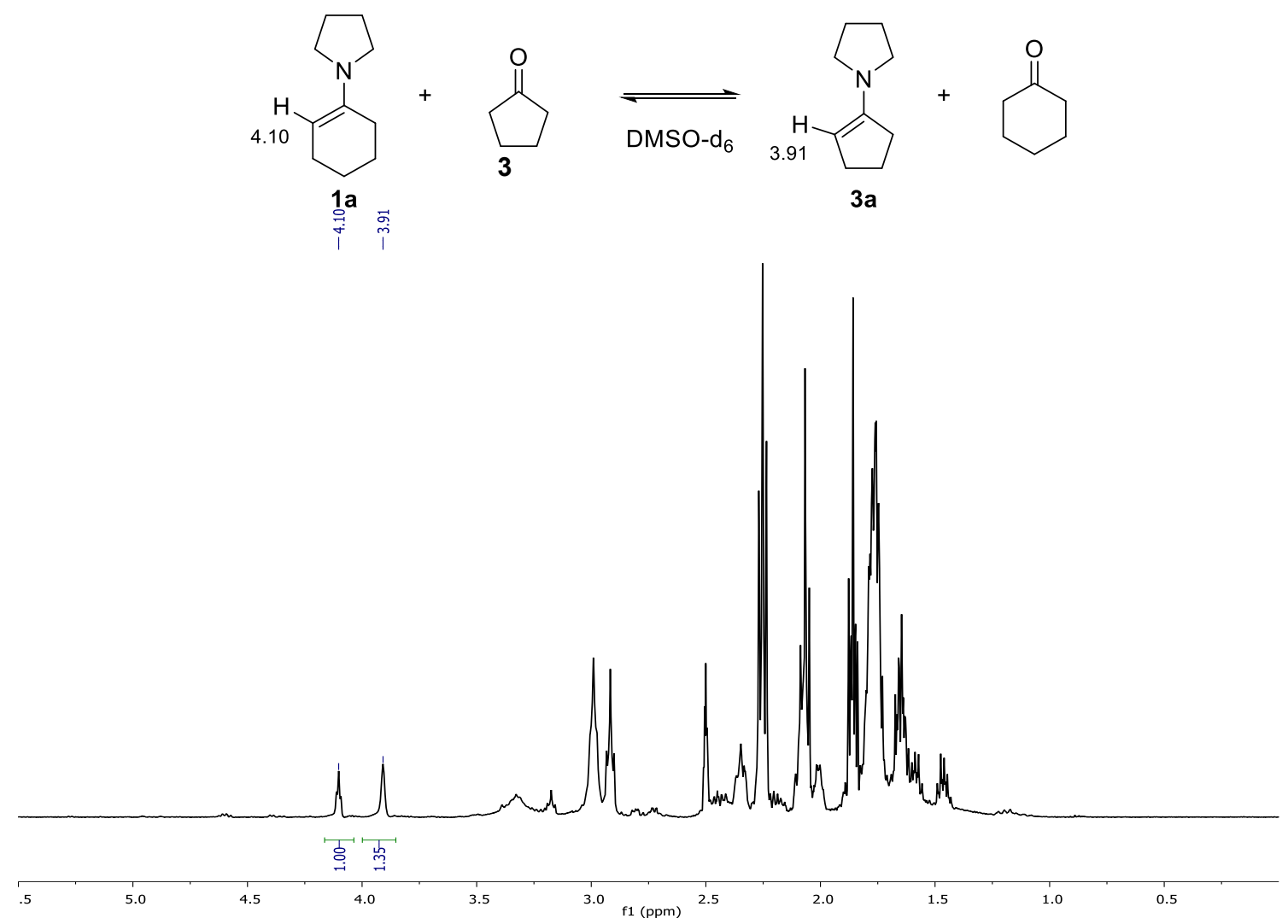
${ }^{1}$ H NMR Spectrum of Enamine 1c

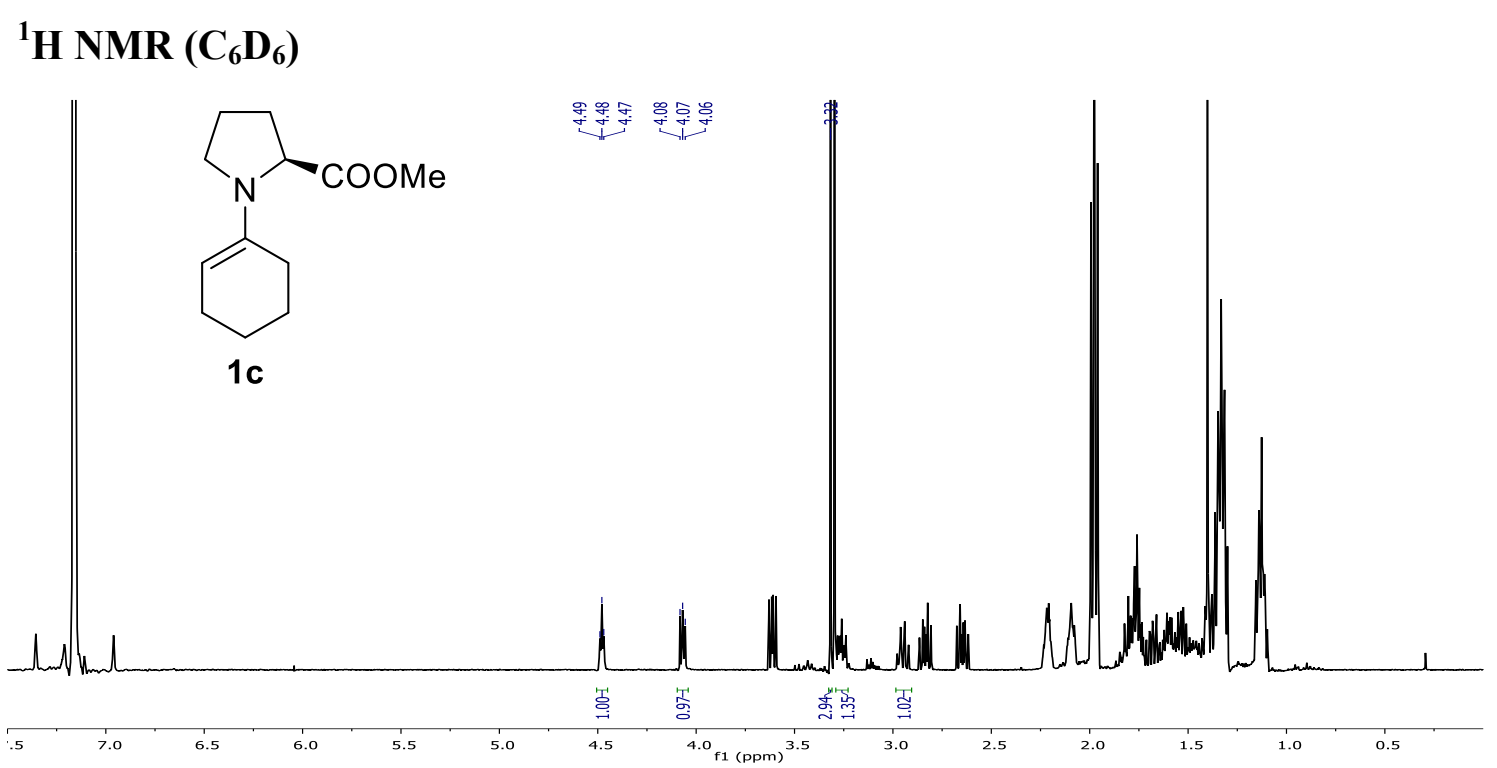

Enamine 1c, relevant signals: $\delta 4.48(\mathrm{t}, J=3.9 \mathrm{~Hz}, 1 \mathrm{H}), 4.07(\mathrm{~m}, 1 \mathrm{H}), 3.32(\mathrm{~s}, 3 \mathrm{H})$.

\section{Reaction of 1c with Pyrrolidine}

In $\mathrm{C}_{6} \mathrm{D}_{6}\left({ }^{1} \mathrm{H}\right.$ NMR)
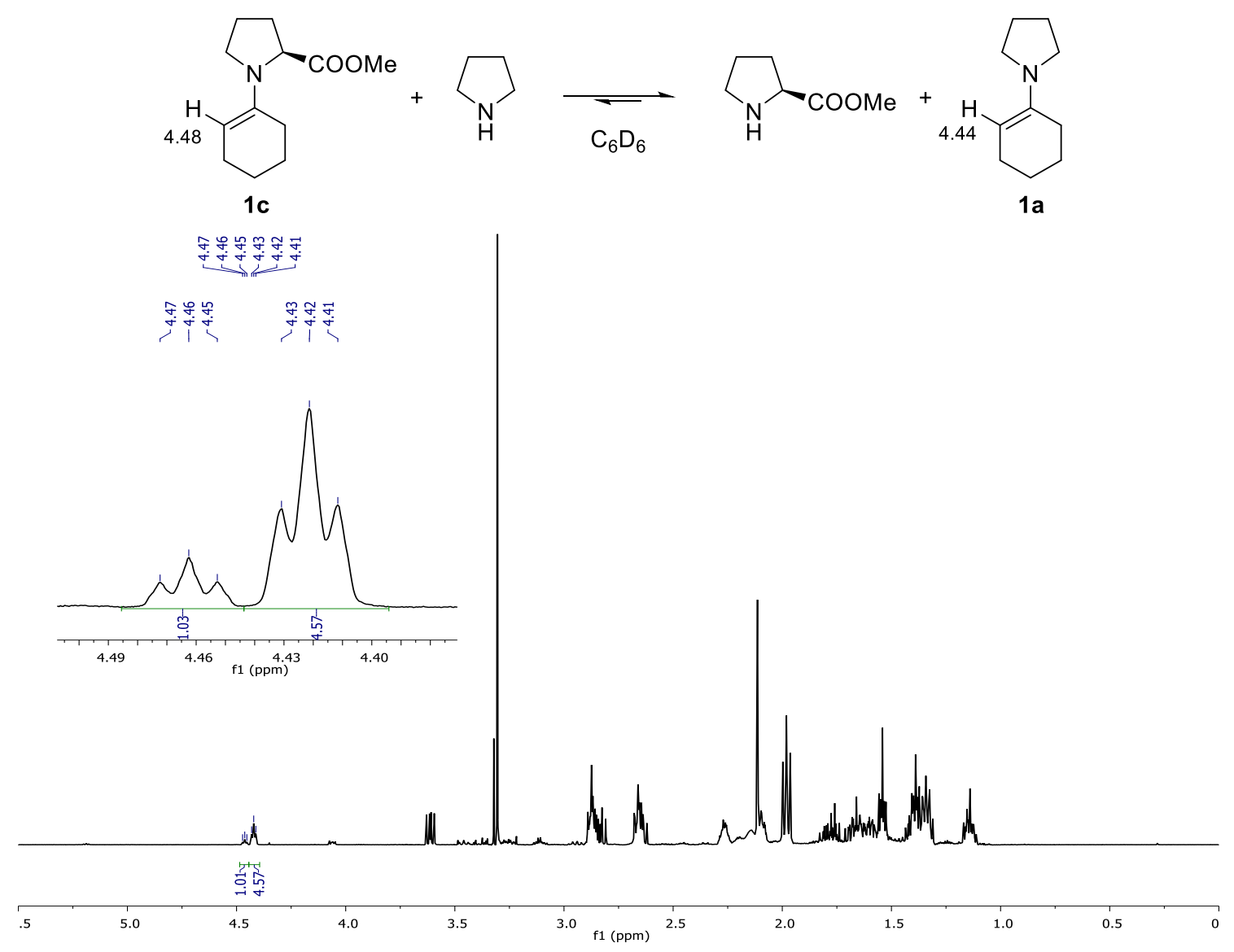
In DMSO- $d_{6}\left({ }^{1} \mathrm{H}\right.$ NMR $)$
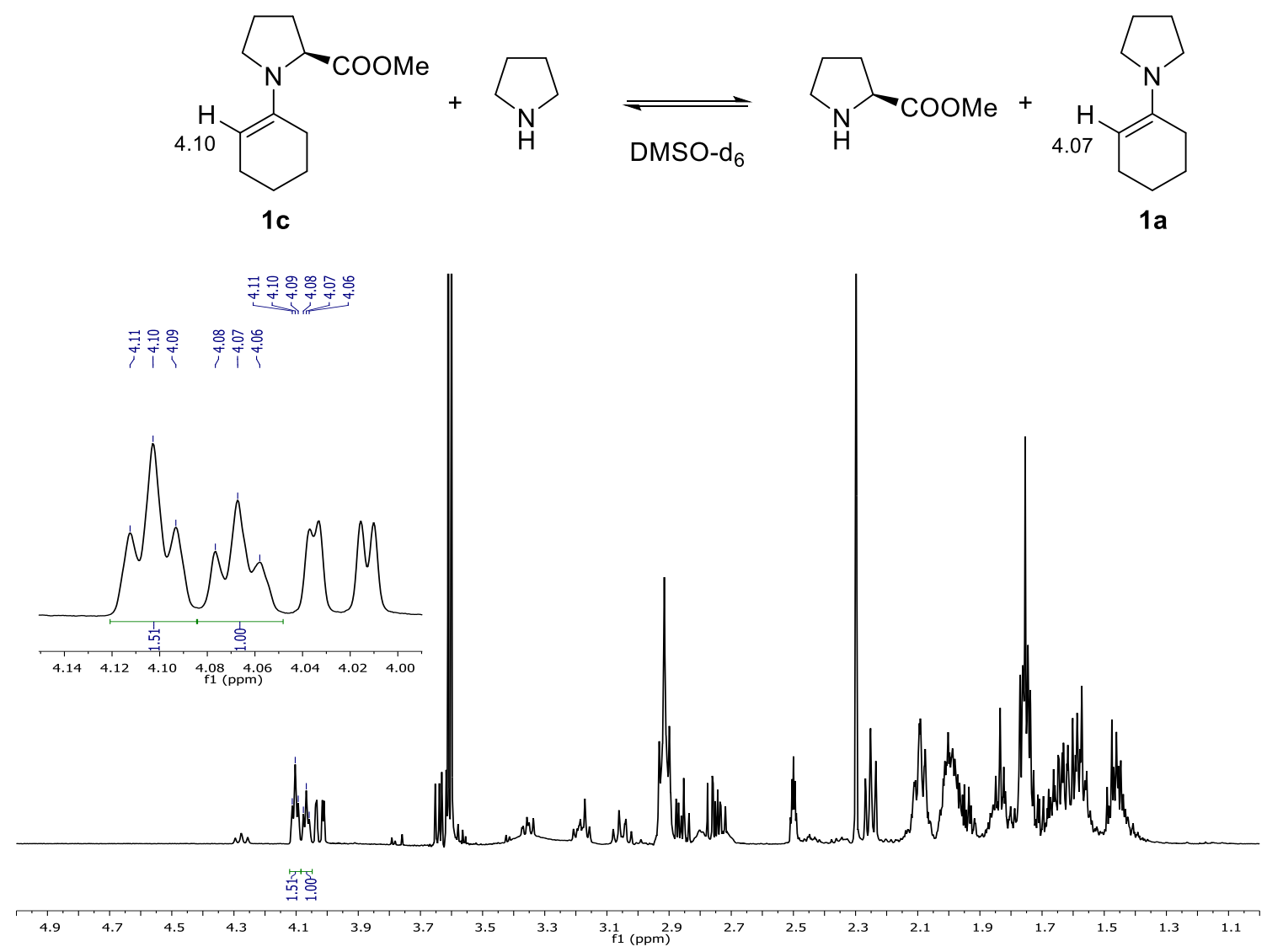

NMR Spectra of Enamine 5a

${ }^{1} \mathrm{H} \operatorname{NMR}\left(\mathrm{C}_{6} \mathbf{D}_{6}\right)$

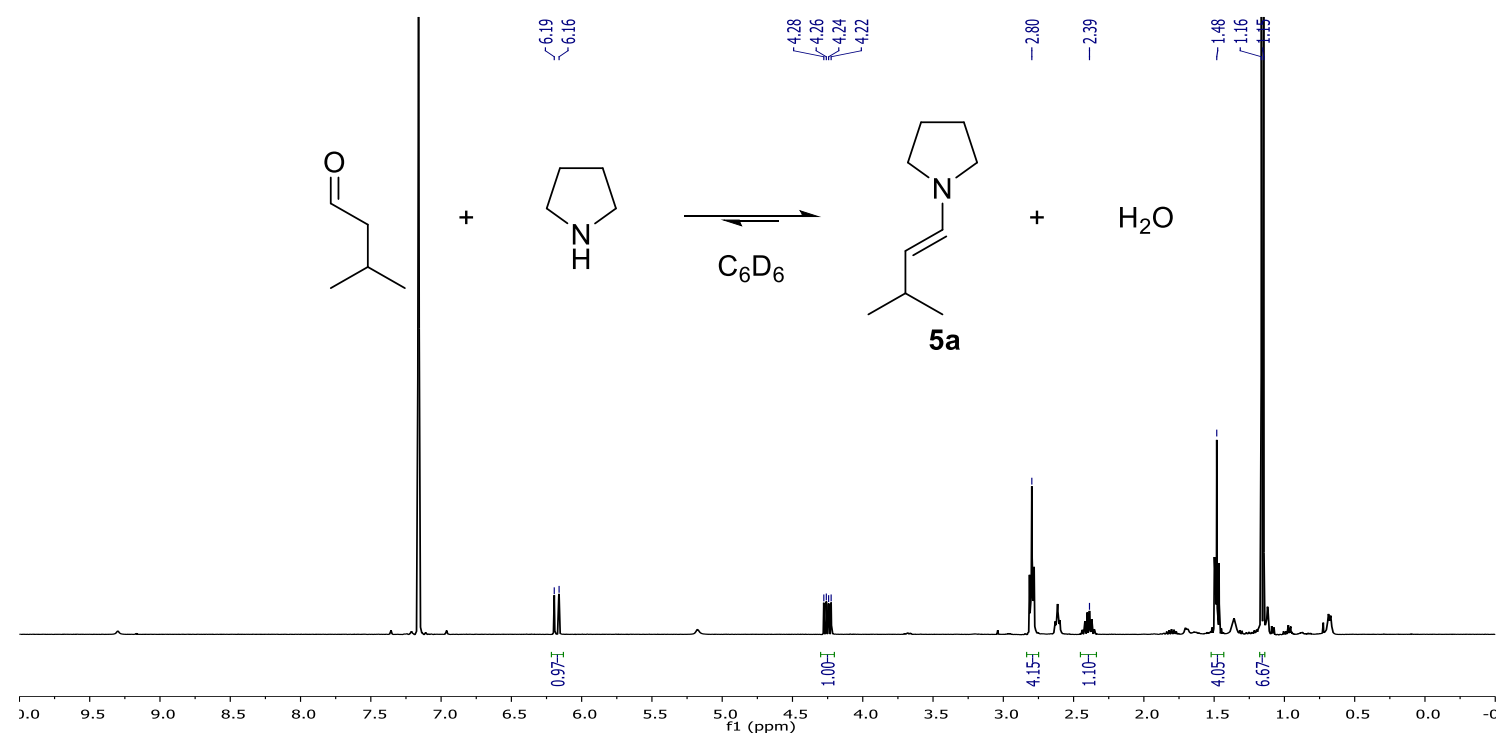

Enamine 5a: $\delta 6.18(\mathrm{~d}, J=13.7 \mathrm{~Hz}, 1 \mathrm{H}), 4.25(\mathrm{dd}, J=13.7,7.2 \mathrm{~Hz}, 1 \mathrm{H}), 2.80(\mathrm{~m}, 4 \mathrm{H})$, $2.39(\mathrm{~m}, 1 \mathrm{H}), 1.48(\mathrm{~m}, 4 \mathrm{H}), 1.16(\mathrm{~d}, J=6.7 \mathrm{~Hz}, 6 \mathrm{H})$. 


\section{${ }^{1}$ H NMR (DMSO-d $)_{6}$}
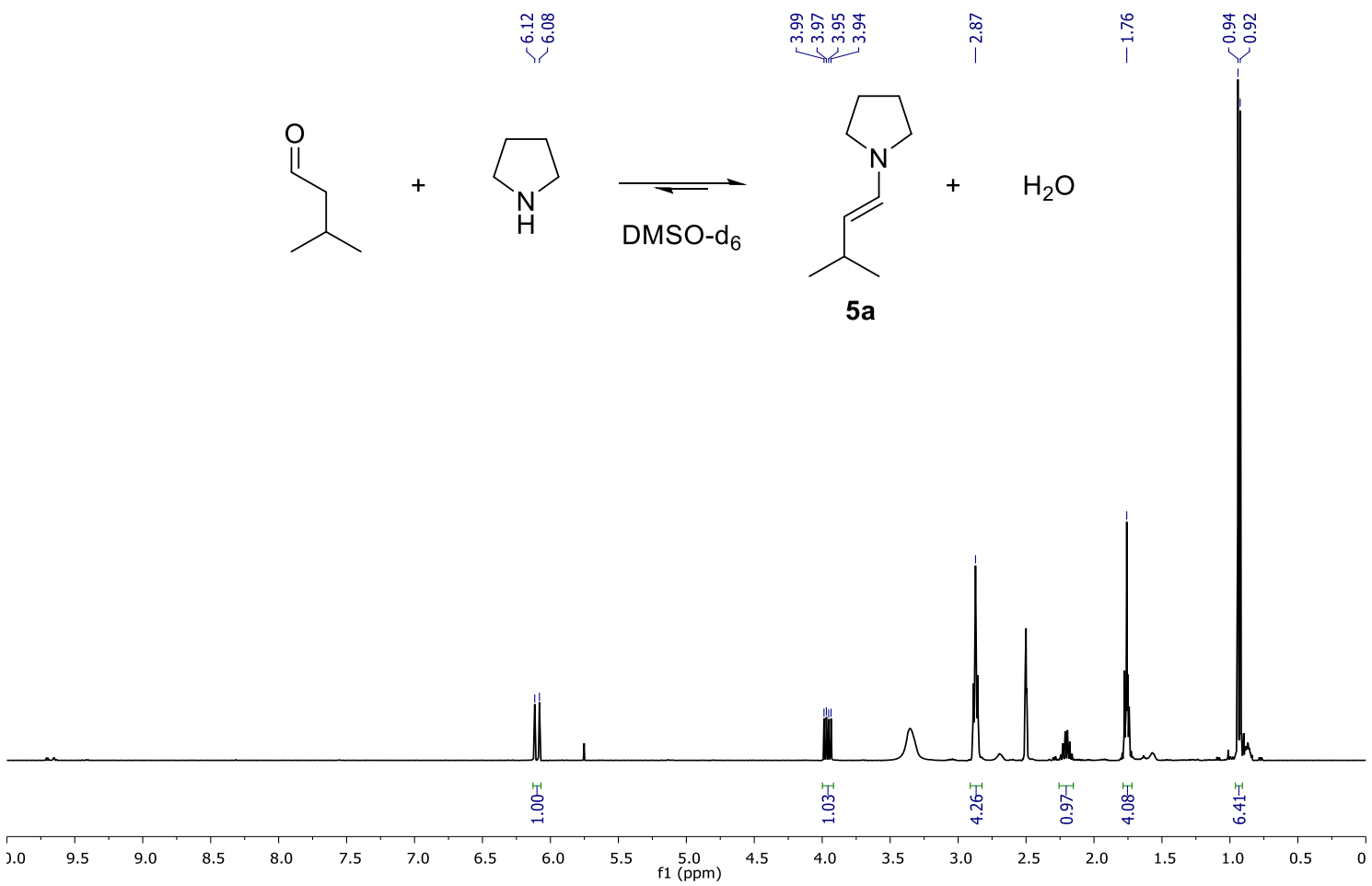

Enamine 5a: $\delta 6.14(\mathrm{~d}, J=13.8 \mathrm{~Hz}, 1 \mathrm{H}), 4.12(\mathrm{dd}, J=13.8,7.0 \mathrm{~Hz}, 1 \mathrm{H}), 2.94(\mathrm{~m}, 4 \mathrm{H})$, $2.25(\mathrm{~m}, 1 \mathrm{H}), 1.82(\mathrm{~m}, 4 \mathrm{H}), 0.97(\mathrm{~d}, J=6.7 \mathrm{~Hz}, 6 \mathrm{H})$.

\section{${ }^{13}$ C NMR (DMSO-d $)$}
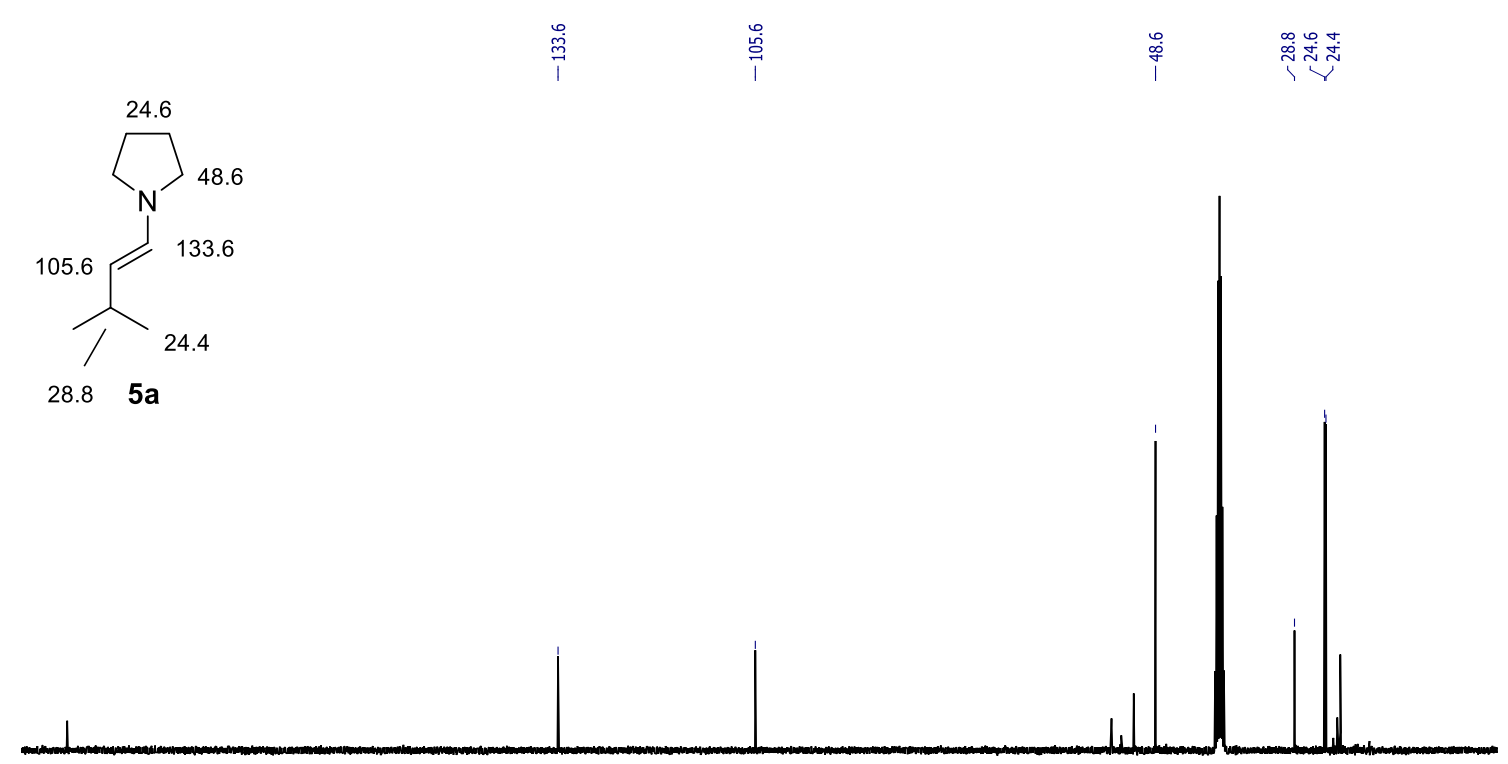

$\begin{array}{lllllllllll}10 & 200 & 190 & 180 & 170 & 160 & 150 & 140 & 130 & 120 & \substack{110 \\ \mathrm{f} 1(\mathrm{ppm})} \\ 100 & 100 & & & & & & & & & \end{array}$

Enamine 5a: $\delta$ 133.6, 105.6, 48.6, 28.8, 24.6, 24.4. 


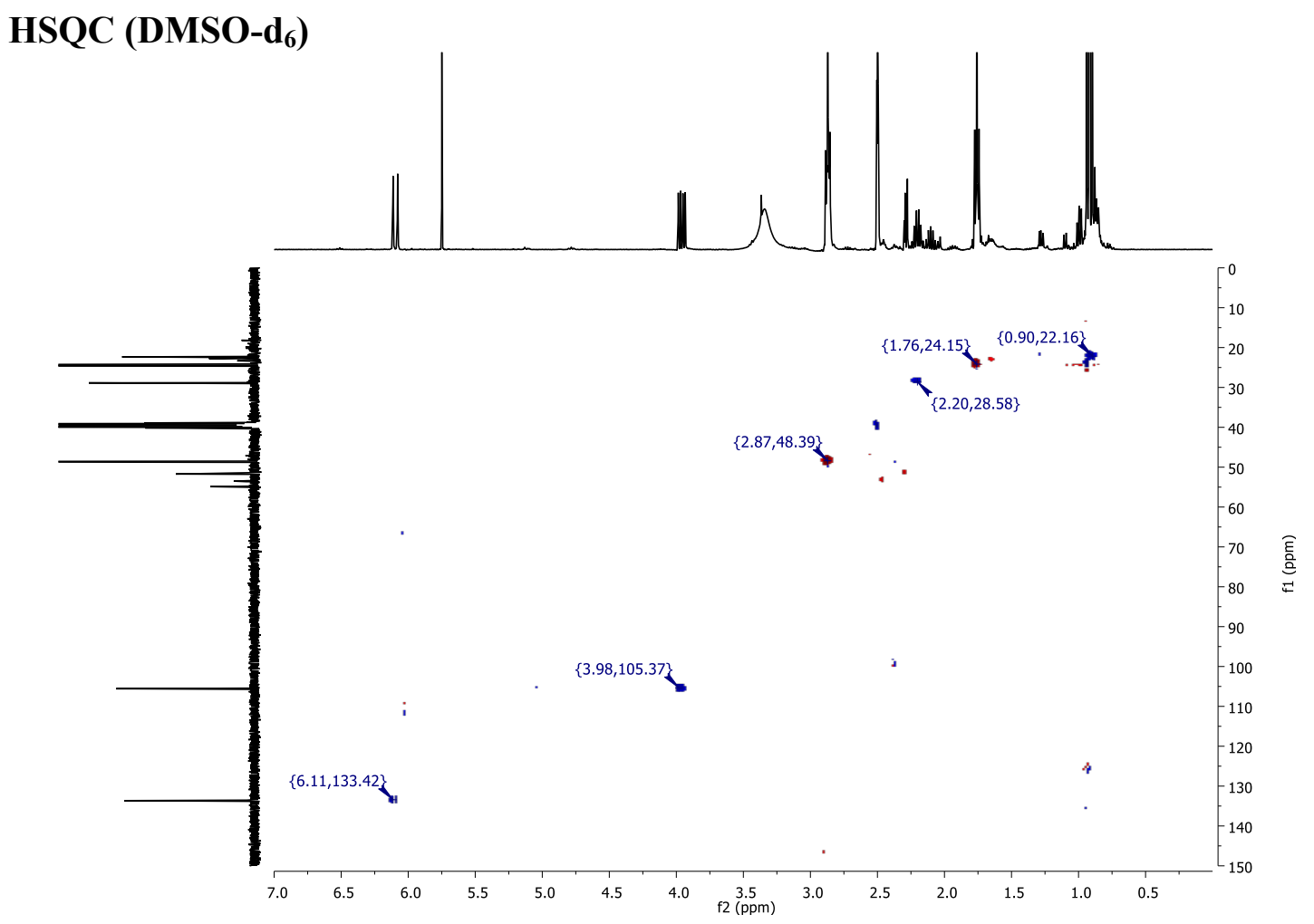

Equilibrium between 5a and 5aa in DMSO- $d_{6}$<smiles>CC(C)/C=C/N1CCCC1</smiles>

$5 a$<smiles>C1CCNC1</smiles>

DMSO-d $_{6}$

${ }^{1}$ H NMR

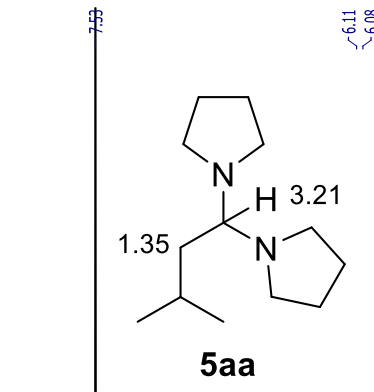

궁<smiles>CC(C)CC(N1CCCC1)N1CCCC1</smiles>

5 aa<smiles>Brc1ccc(Br)cc1</smiles>
. 

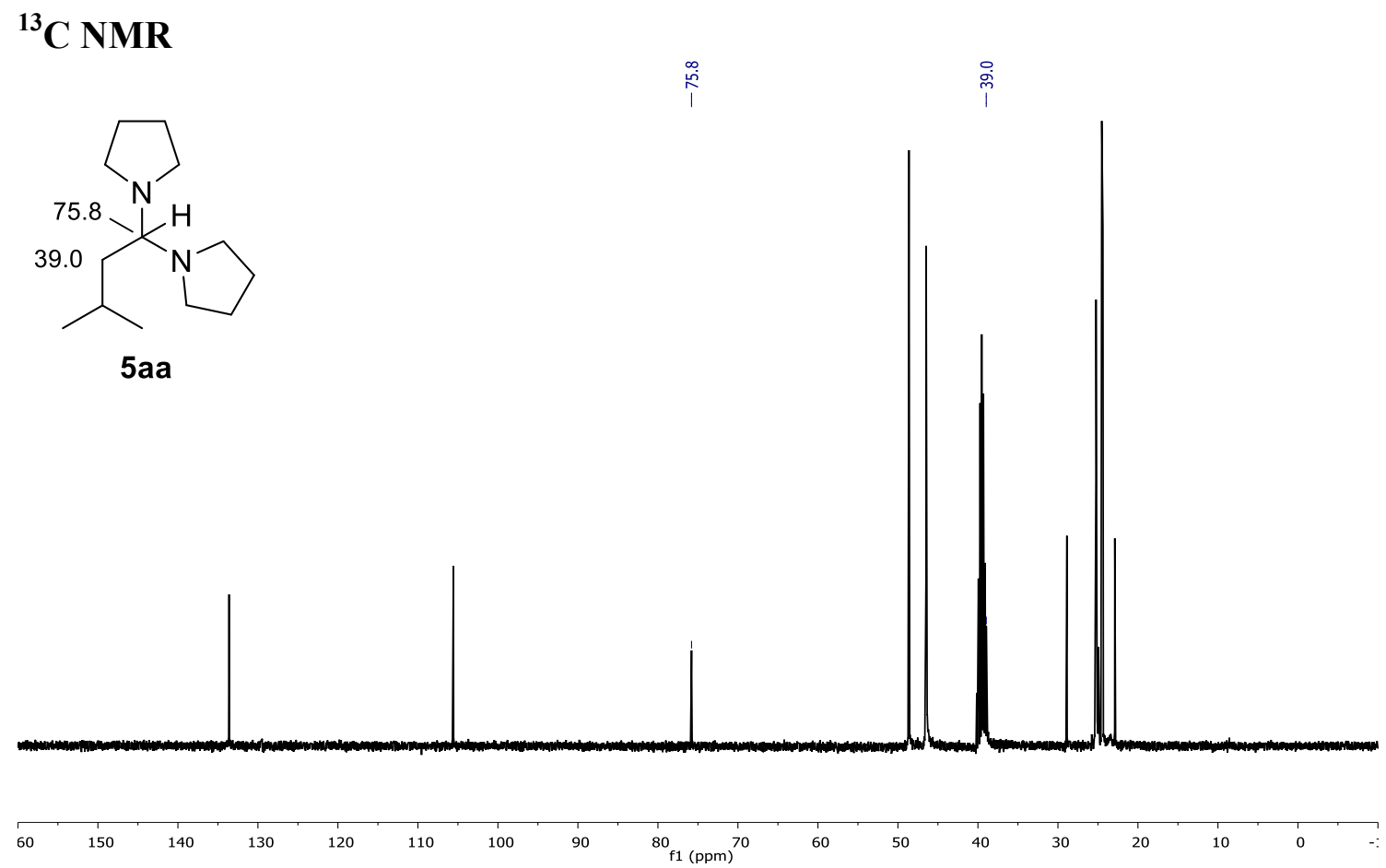

Aminal 5aa, relevant signals: $\delta$ 75.8, 39.0. Peaks at $\delta 133.6$ and 105.6 are due to 5a.

\section{HSQC}

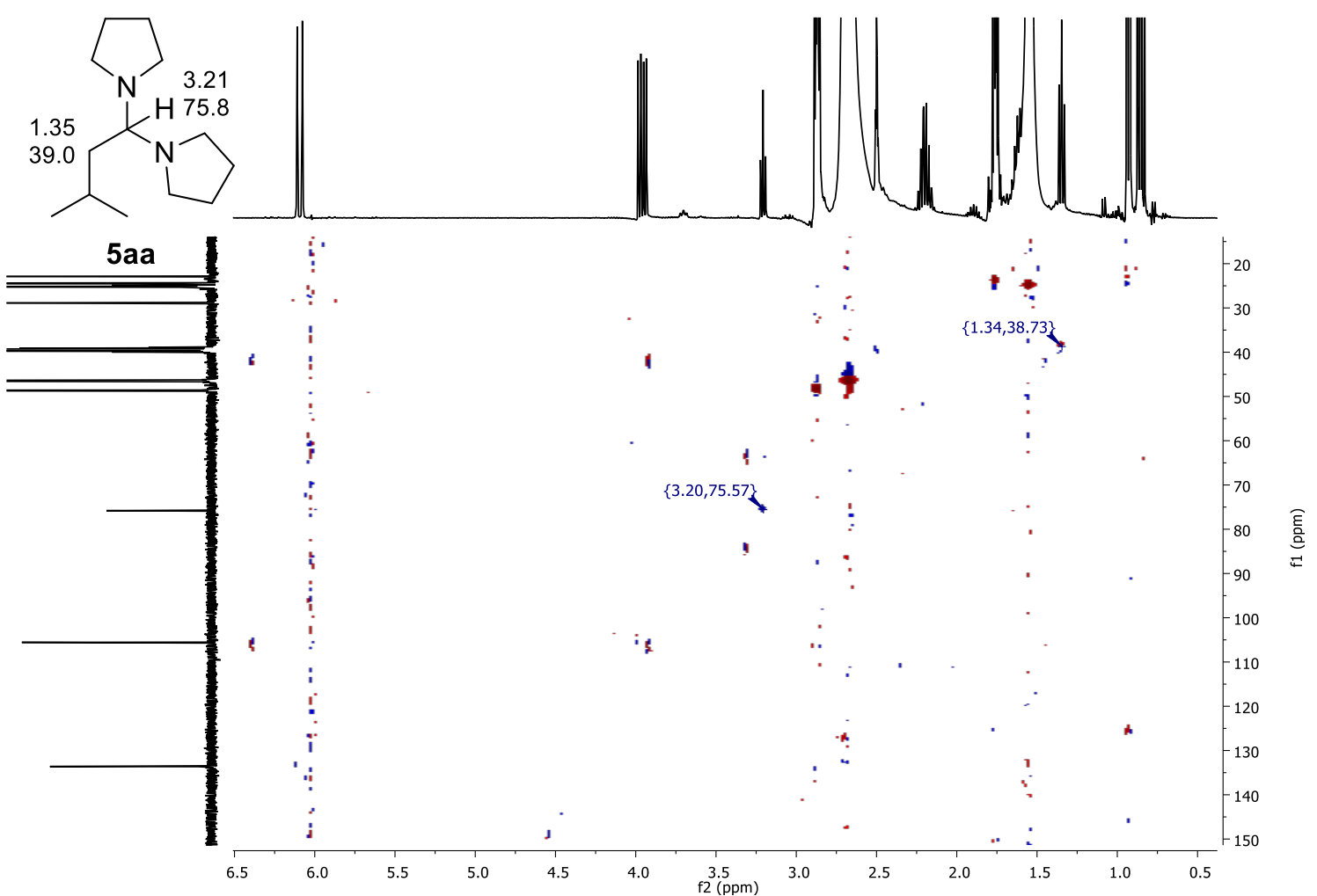


Equilibrium between $5 \mathrm{a}$ and $5 \mathrm{aa}$ in $\mathrm{C}_{6} \mathrm{D}_{6}$
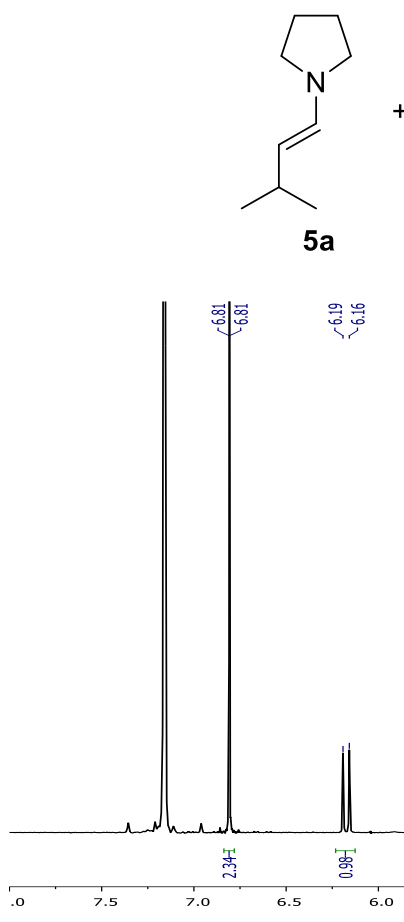<smiles>C1CCNC1</smiles>

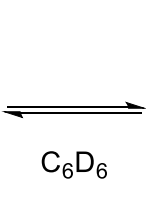

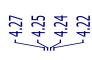<smiles>CC(C)CC(N1CCCC1)N1CCCC1</smiles>

5aa<smiles>Brc1ccc(Br)cc1</smiles>

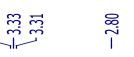

Aminal 5aa, relevant signal: $\delta 3.33$ (t, $J=6.4 \mathrm{~Hz}, 1 \mathrm{H})$. A weighted amount of 1,4-dibromobenzene $\left(\delta 6.81\right.$ in $\left.\mathrm{C}_{6} \mathrm{D}_{6}\right)$ was added as an internal standard in order to determine the concentrations of all species in the equilibrium.

\section{Enamine 5b}

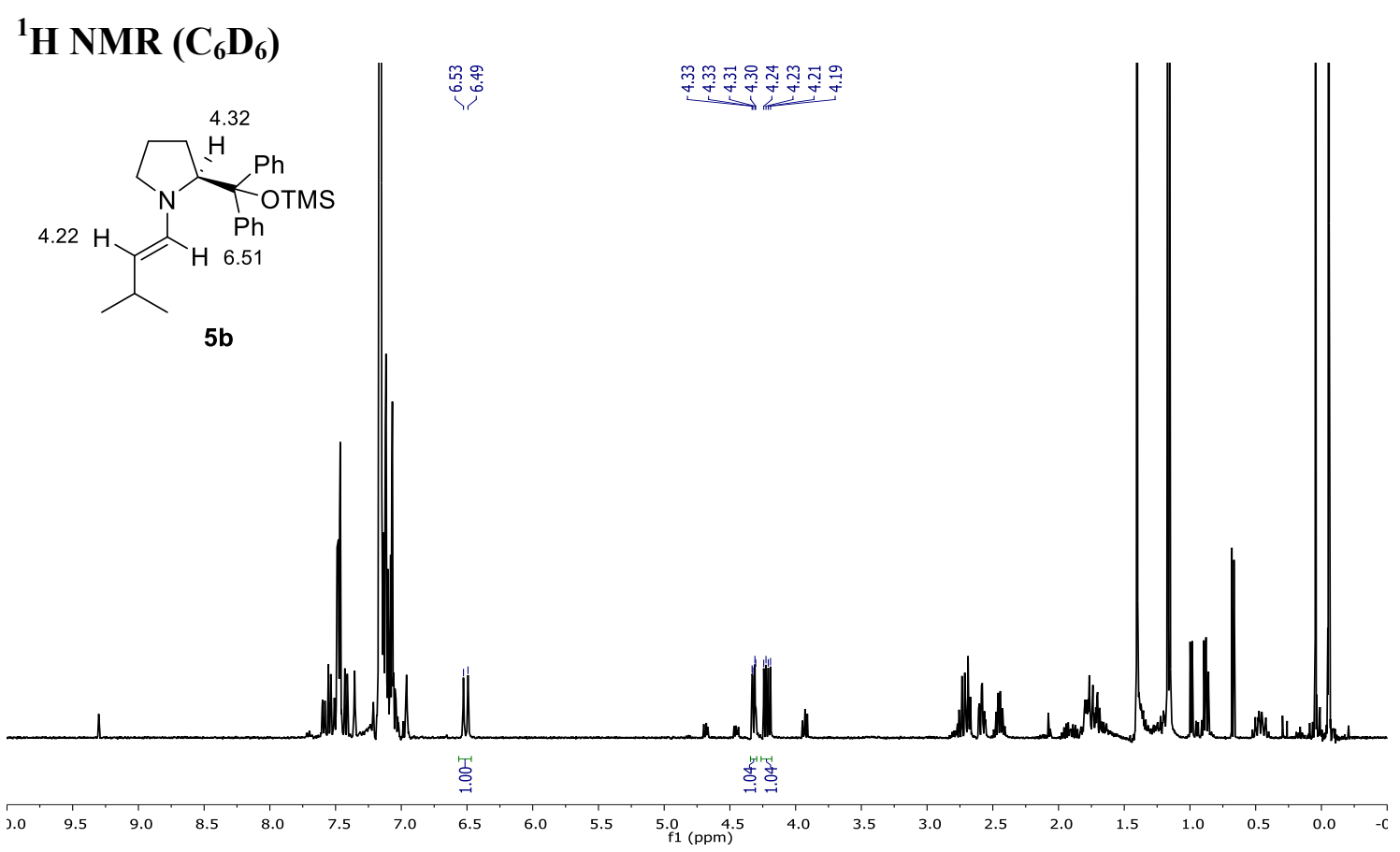

Enamine 5b, relevant signals: $\delta 6.51(\mathrm{~d}, J=13.9 \mathrm{~Hz}, 1 \mathrm{H}), 4.32(\mathrm{dd}, J=8.7,2.3 \mathrm{~Hz}$, $1 \mathrm{H}), 4.22$ (dd, $J=13.7,6.8 \mathrm{~Hz}, 1 \mathrm{H})$. 
Reaction of $5 \mathrm{~b}$ with pyrrolidine in $\mathrm{C}_{6} \mathrm{D}_{6}$

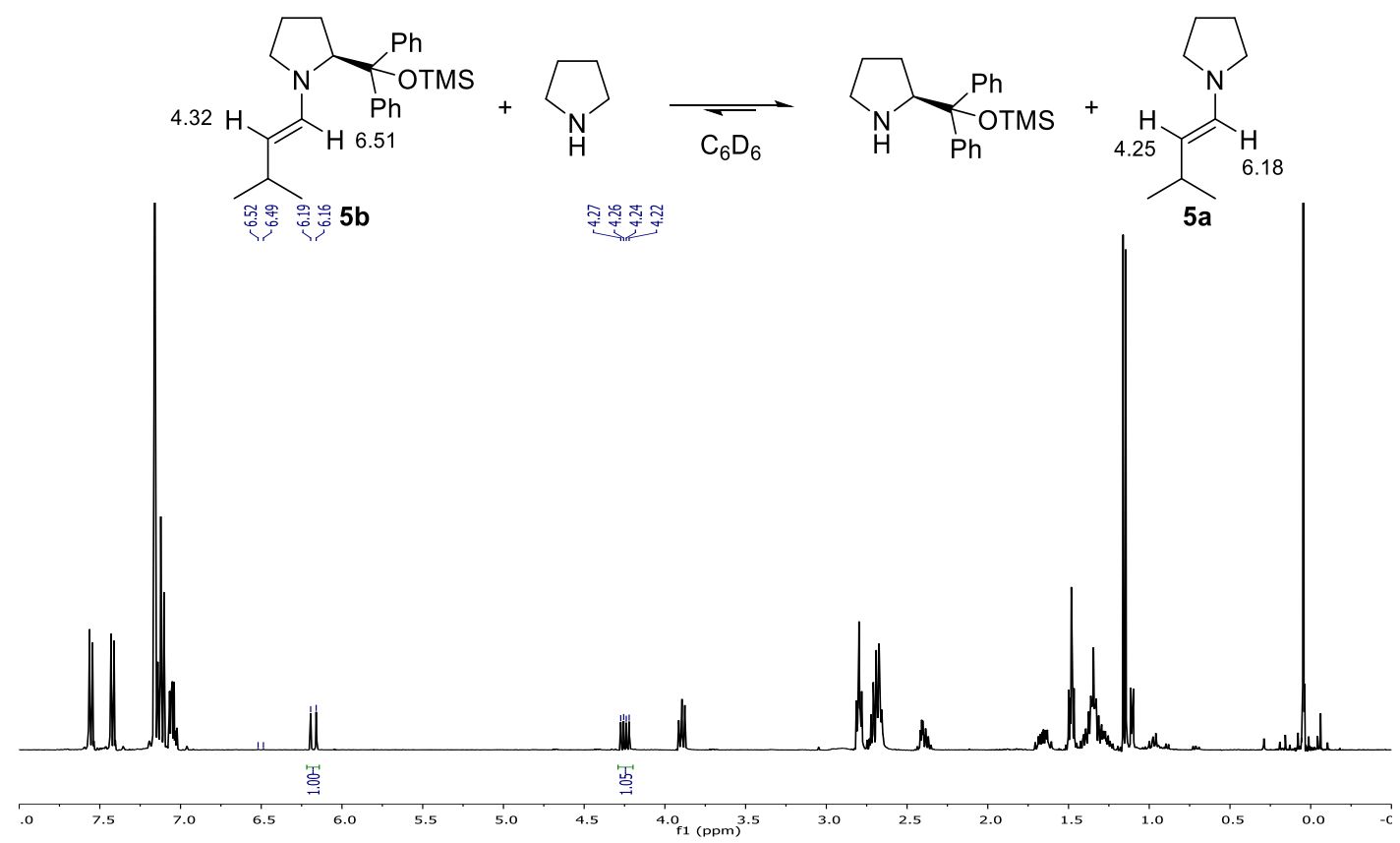

Hemiaminal from 7' and pyrrolidine

\section{${ }^{1} \mathrm{H}$ NMR $\left(\mathbf{C}_{6} \mathbf{D}_{6}\right)$}

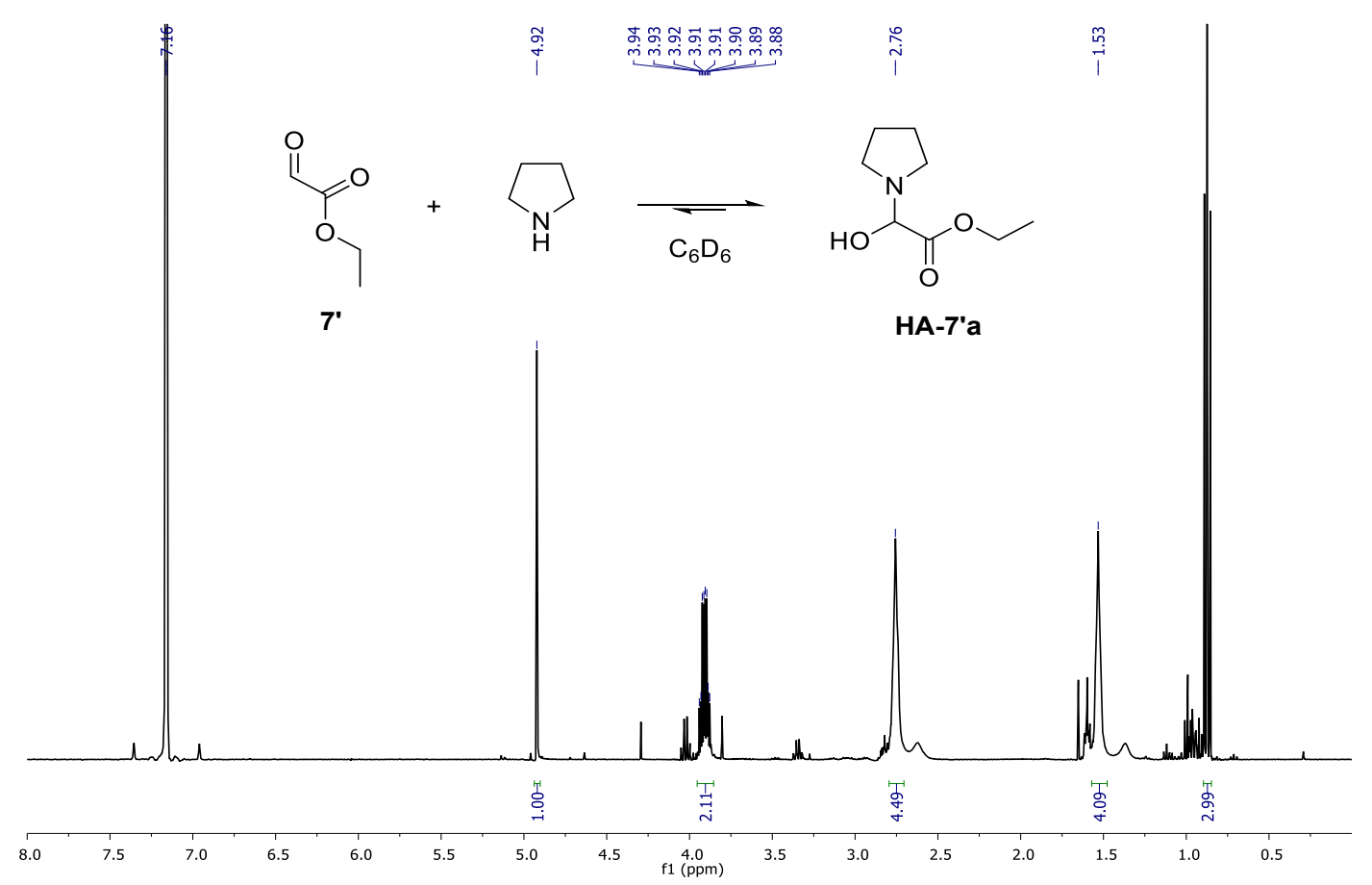

HA-7'a: $\delta 4.92(\mathrm{~s}, 1 \mathrm{H}), 3.91(\mathrm{q}, J=7.1 \mathrm{~Hz}, 2 \mathrm{H}), 2.76(\mathrm{~m}, 4 \mathrm{H}), 1.53(\mathrm{~m}, 4 \mathrm{H}), 0.87(\mathrm{t}, J=$ $7.1 \mathrm{~Hz}, 3 \mathrm{H}$ ). Expansion of the signals centered at $3.91 \mathrm{ppm}$ indicates a split quadruplet (an $\mathrm{ABX}_{3}$ system, with $v_{\mathrm{A}}-v_{\mathrm{B}}=3.8 \mathrm{~Hz}$, likely due to the presence of the stereocenter and the $\mathrm{OH} \cdots \mathrm{O}$ hydrogen bond). 


\section{${ }^{13} \mathrm{C}$ NMR $\left(\mathrm{C}_{6} \mathrm{D}_{6}\right)$}

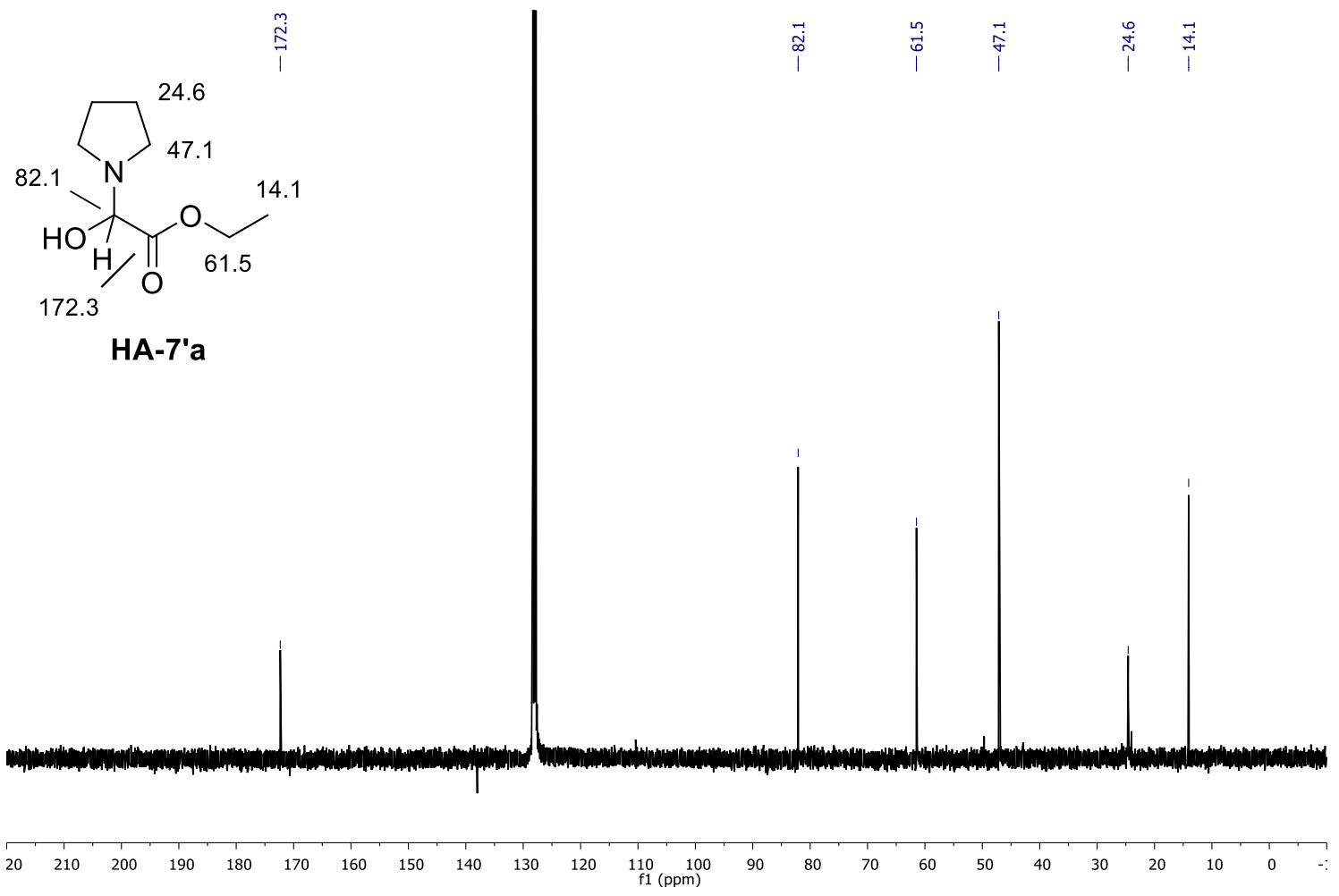

HA-7'a: $\delta 172.3,82.1,61.5,47.1,24.6,14.1$.

\section{HSQC $\left(C_{6} D_{6}\right)$}

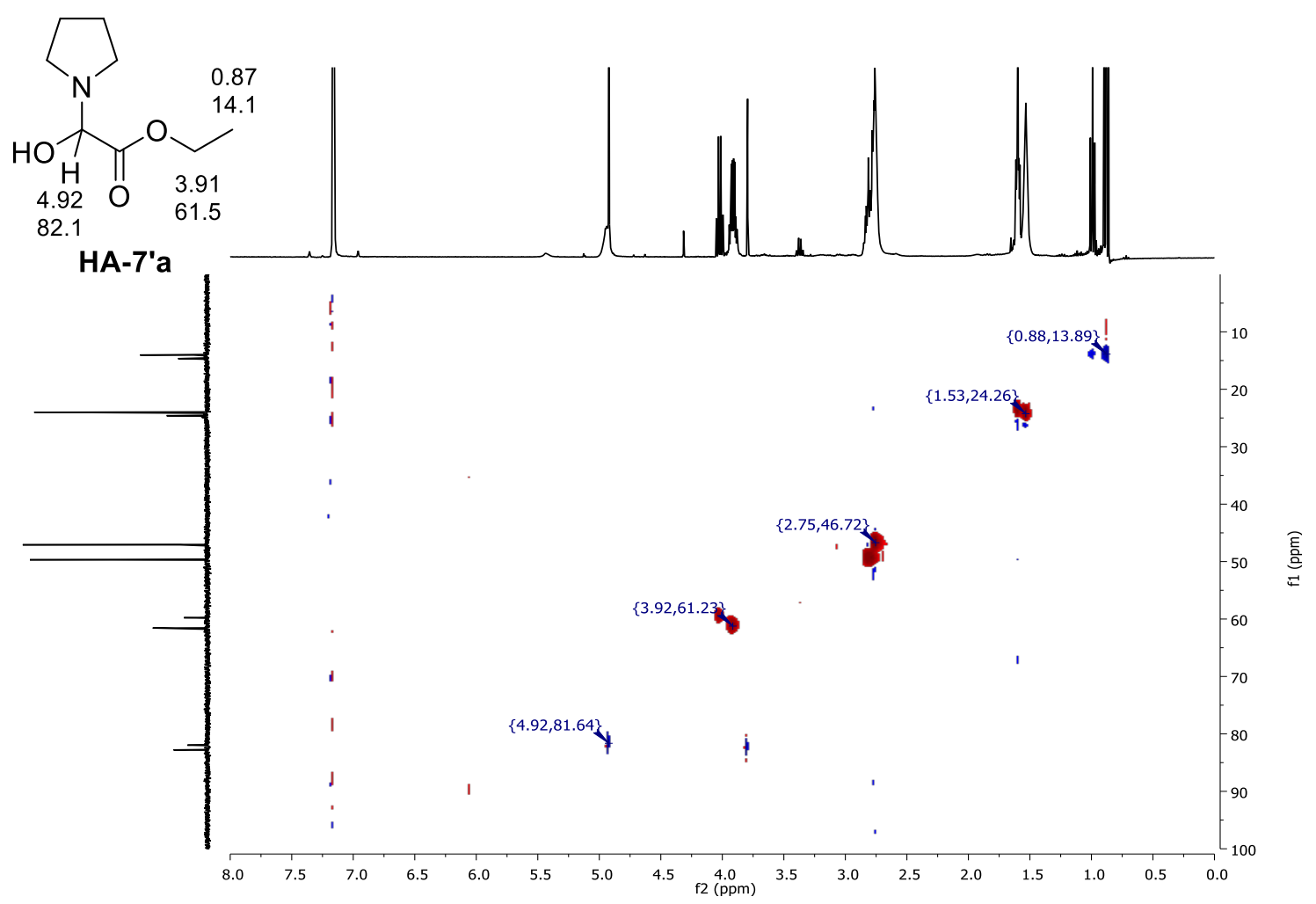

During this HSQC experiment, signals due to 7'aa began to appear. 
${ }^{1}$ H NMR (DMSO-d $)$

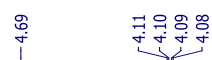

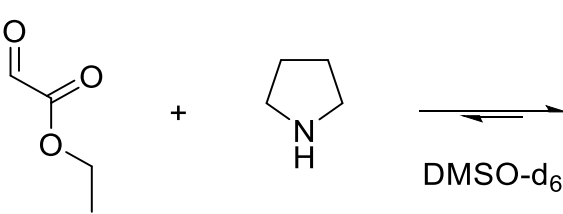

$7^{\prime}$
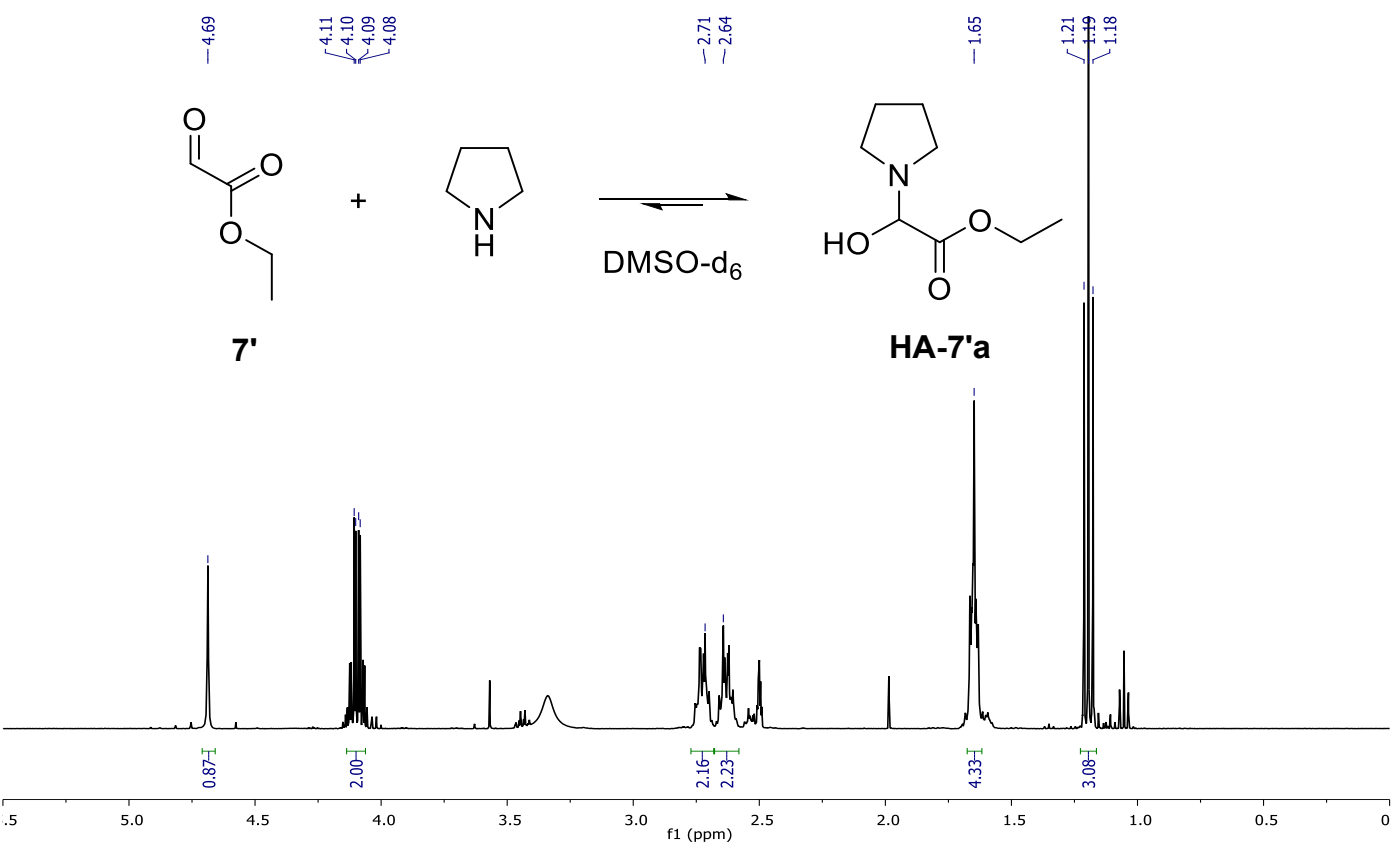

HA-7'a: $\delta 4.69$ (s, 1H), 4.09 (q, $J=7.1 \mathrm{~Hz}, 2 \mathrm{H}), 2.73(\mathrm{~m}, 2 \mathrm{H}), 2.63(\mathrm{~m}, 2 \mathrm{H}), 1.65(\mathrm{~m}, 4 \mathrm{H})$, $1.19(\mathrm{t}, J=7.1 \mathrm{~Hz}, 3 \mathrm{H})$.

\section{Aminal 7'aa}

${ }^{1} \mathrm{H} \operatorname{NMR}\left(\mathrm{C}_{6} \mathbf{D}_{6}\right)$

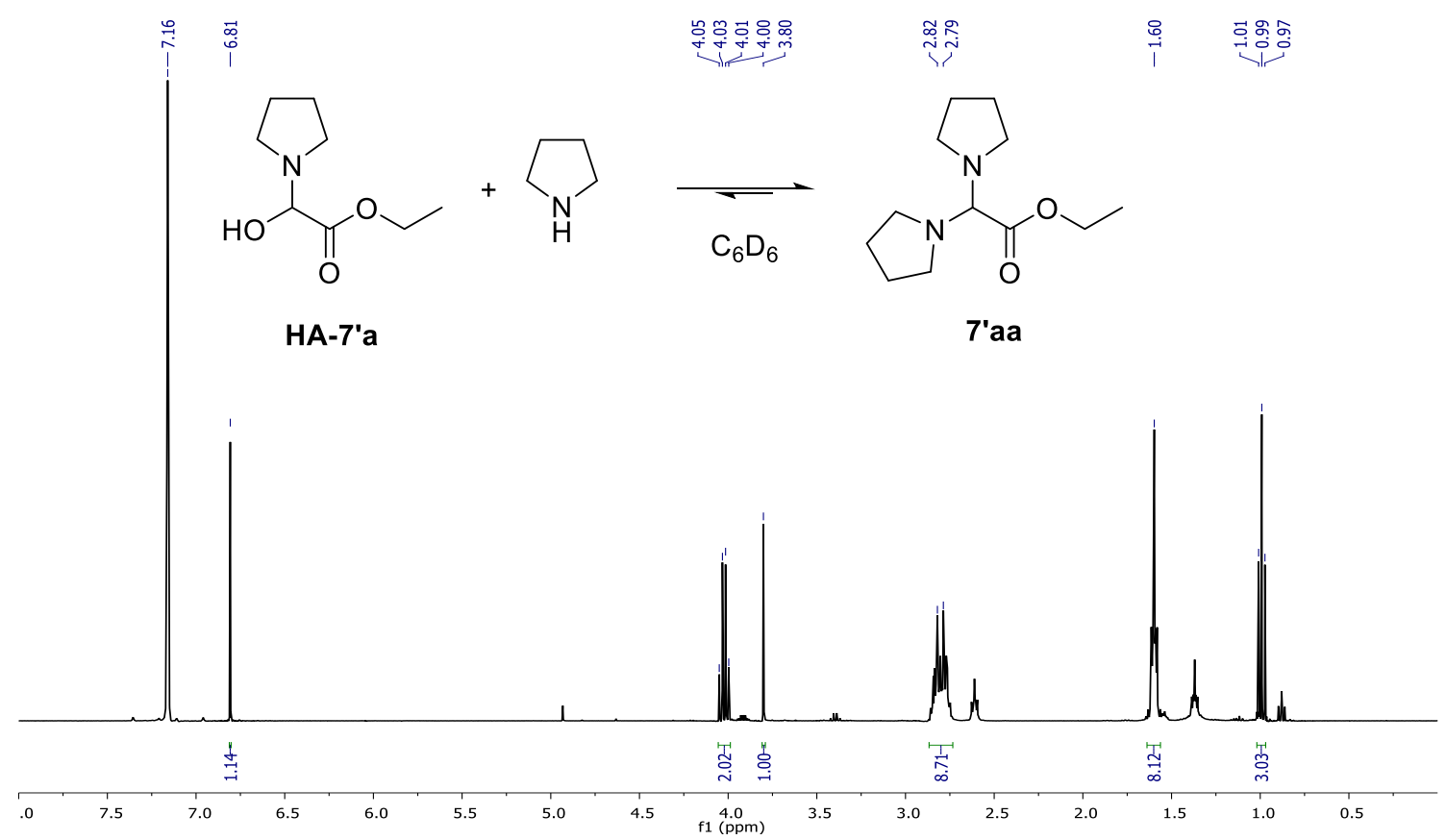

7'aa: $\delta 4.02(\mathrm{q}, J=7.2 \mathrm{~Hz}, 2 \mathrm{H}), 3.80(\mathrm{~s}, 1 \mathrm{H}), 2.89-2.72(\mathrm{~m}, 8 \mathrm{H}), 1.60(\mathrm{~m}, 8 \mathrm{H}), 0.99(\mathrm{t}, J=$ $7.1 \mathrm{~Hz}, 3 \mathrm{H})$. A weighted amount of 1,4-dibromobenzene $\left(\delta 6.81\right.$ in $\left.\mathrm{C}_{6} \mathrm{D}_{6}\right)$ was added as an internal standard to determine the true concentration of all the species in the equilibrium. 
${ }^{13} \mathrm{C}$ NMR $\left(\mathrm{C}_{6} \mathrm{D}_{6}\right)$
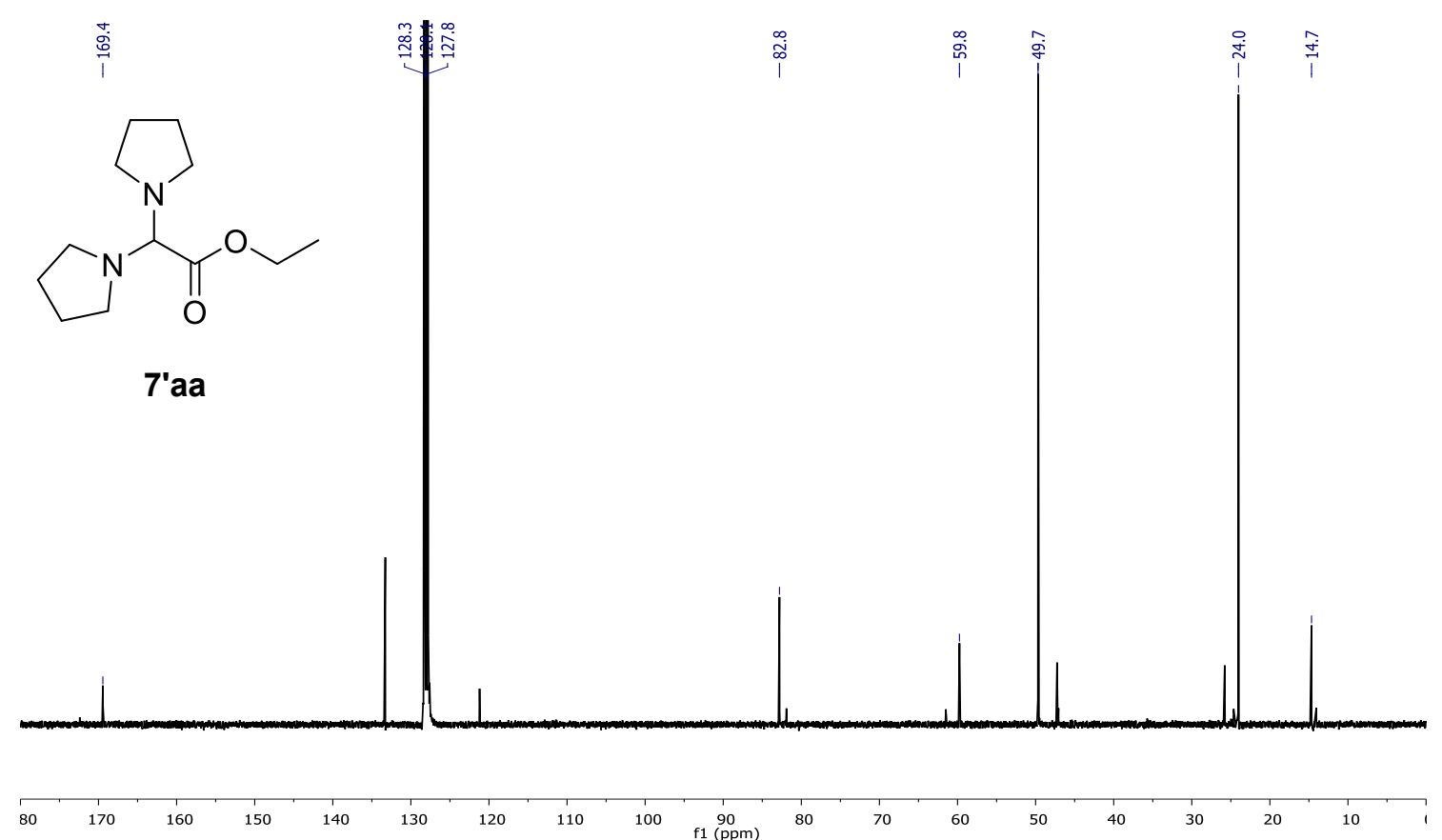

7'aa: $\delta 169.4,82.8,59.8,49.7,24.0,14.7$. The solution contained 1,4-dibromobenzene $\left(\delta \mathrm{CH} 133.3\right.$ and $\delta \mathrm{C}_{\mathrm{q}} 121.2$ in $\left.\mathrm{C}_{6} \mathrm{D}_{6}\right)$.

\section{$\operatorname{HSQC}\left(\mathrm{C}_{6} \mathrm{D}_{6}\right)$}

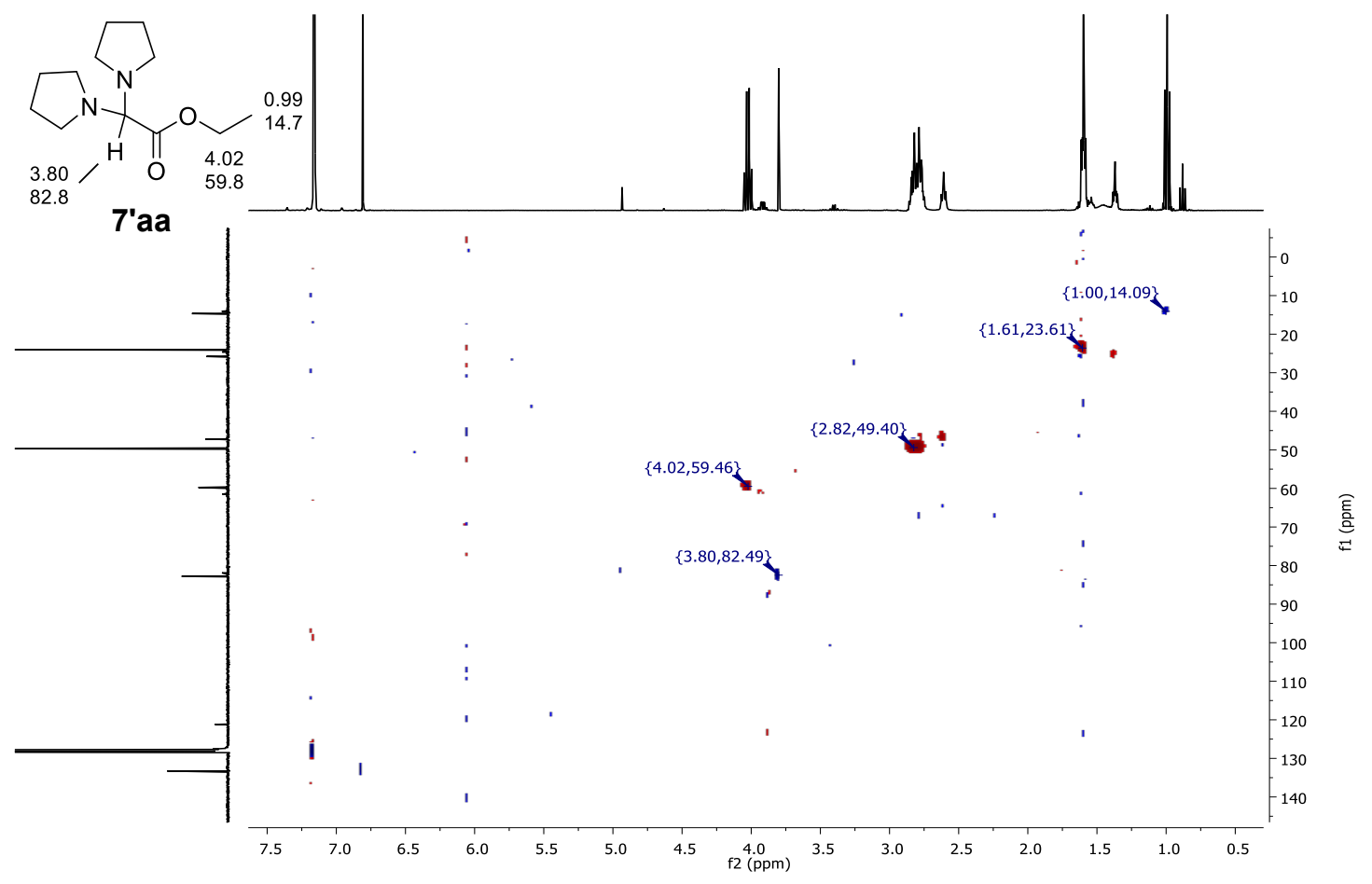




\section{Representative example: formation of $5 a$ in $C_{6} D_{6}$}

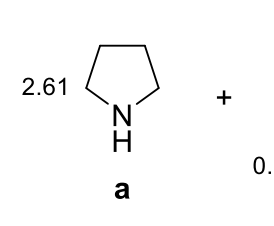<smiles>CC(C)CC=O</smiles><smiles>Oc1ccccc1</smiles><smiles>CC(C)/C=C\[18OH]</smiles>

$5 a$

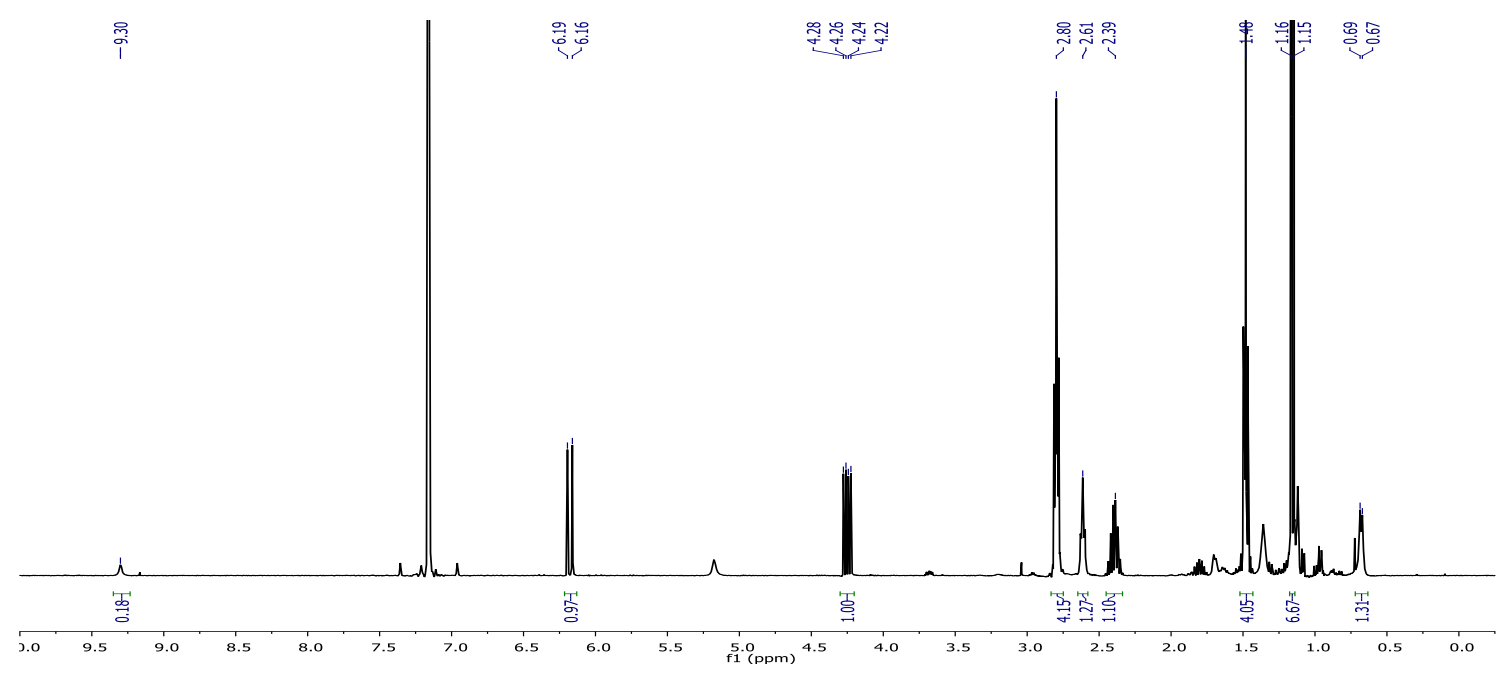

Under rigorously anhydrous conditions (anhydrous solvent, pyrrolidine and $\mathbf{5}$ stored over molecular sieves, 'flame-dried' NMR tube, etc.), the number of mmol of water generated must be equal to that of 5a. Although it is difficult to accomplish in practice, it may be assumed that $\left[\mathrm{H}_{2} \mathrm{O}\right] \approx[\mathbf{5 a}]$ at the equilibrium position. Therefore, approximately:

$$
K_{e q, 5 a}=\frac{[\mathbf{5 a}] \cdot[\mathbf{5 a}]}{[\mathbf{5}] \cdot[\boldsymbol{a}]}=\frac{1.00^{2}}{\frac{1.31}{6} \cdot \frac{1.27}{4}}=14.4
$$




\section{Representative example: formation of $\mathrm{HA}-7^{\prime} \mathrm{b}$ in $\mathrm{C}_{6} \mathrm{D}_{6}$}

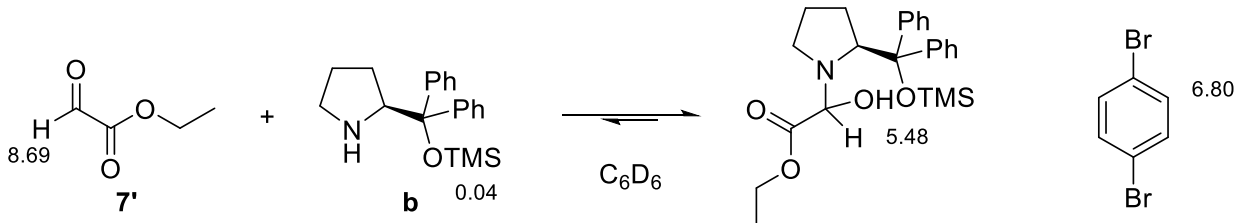

HA-7'b

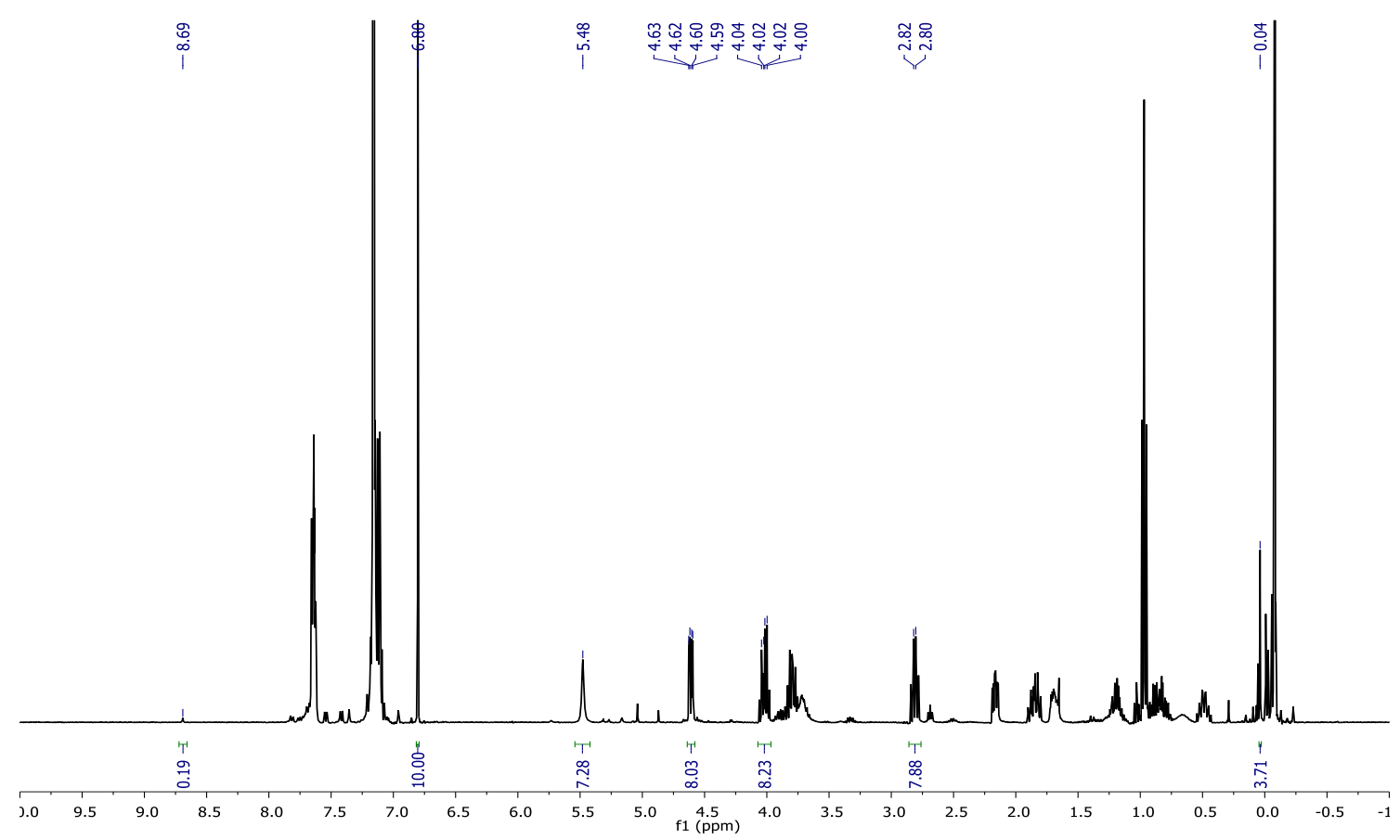

Equimolar amounts of aldehyde 7' (ethyl glyoxylate, or ethyl 2-oxoacetate, the commercially available homologue of methyl glyoxylate, 7$)$ and catalyst $\mathbf{b}(0.060 \mathrm{mmol})$ were mixed in $\mathrm{C}_{6} \mathrm{D}_{6}$ $(0.70 \mathrm{~mL})$. A weighted amount of 1,4 -dibromobenzene $(0.021 \mathrm{mmol})$ was added $\left(\delta 6.80\right.$ in $\left.\mathrm{C}_{6} \mathrm{D}_{6}\right)$. Once the equilibrium was reached, the concentrations of the chemical species were determined on the basis of the known concentration of 1,4-dibromobenzene and the relative integration of appropriate signals.

Aldehyde 7'

Hemiaminal HA-7'b

Jørgensen-Hayashi catalyst (b)

$$
\begin{array}{llr}
\delta 8.69(1 \mathrm{H}) & = & 1.6 \cdot 10^{-3} \mathrm{mmol} \\
\delta 5.48(1 \mathrm{H}) & = & 61.0 \cdot 10^{-3} \mathrm{mmol} \\
\delta 0.04(\mathrm{TMS}, 9 \mathrm{H}) & = & 3.5 \cdot 10^{-3} \mathrm{mmol}
\end{array}
$$

$$
K_{e q}=\frac{\left[\mathbf{H A}-\mathbf{7}^{\prime} \mathbf{b}\right]}{\left[\mathbf{7}^{\prime}\right] \cdot[\mathbf{b}]}=\frac{61.0 \cdot 10^{-3} / \mathrm{V}}{1.6 \cdot 10^{-3} / \mathrm{V} \cdot 3.5 \cdot 10^{-3} / \mathrm{V}}=7.6 \cdot 10^{3} \mathrm{M}^{-1}
$$

